\title{
UNDERSTANDING AND EXPLOITING THE RESPONSE OF EHEC O157:H7 TO HUMAN GASTROINTESTINAL CHEMICAL SIGNALS
}

\author{
by \\ Tracy Linda Brittney Lackraj \\ B.Sc., Ryerson University, 2011
}

\author{
A Dissertation \\ Presented to Ryerson University \\ in partial fulfillment of the \\ requirements for the degree of \\ Doctor of Philosophy \\ In the Program of \\ Molecular Science
}

Toronto, Ontario, Canada, 2017

(C) Tracy Linda Brittney Lackraj 2017 


\section{AUTHOR'S DECLARATION}

I hereby declare that I am the sole author of this dissertation. This is a true copy of the dissertation, including any required final revisions, as accepted by my examiners.

I authorize Ryerson University to lend this dissertation to other institutions or individuals for the purpose of scholarly research.

I further authorize Ryerson University to reproduce this dissertation by photocopying or by other means, in total or in part, at the request of other institutions or individuals for the purpose of scholarly research.

I understand that my dissertation may be made electronically available to the public. 


\title{
Understanding and Exploiting the Response of EHEC O157:H7 to Human Gastrointestinal Chemical Signals
}

\author{
Tracy Linda Brittney Lackraj \\ Doctor of Philosophy, 2017 \\ Molecular Science, Ryerson University
}

\begin{abstract}
Enterohemorrhagic Escherichia coli (EHEC) is a clinically relevant foodborne pathogen, resulting in over 95,000 cases of EHEC-associated illness and 60 deaths each year in the US alone. Since EHEC is a continuous global issue with new outbreaks constantly occurring, the development of new therapeutic strategies is vital to minimizing the cases of infection seen each year. A key aspect in new drug development is the identification of vulnerabilities in EHEC's pathogenicity, in particular, during its transit through the human gastrointestinal (GI) tract. As EHEC passes through the human GI tract to its site of colonization in the large intestine, it faces a multitude of host assaults including acute acid stress in the stomach, bile salt stress and cationic antimicrobial peptide exposure in the small intestine, and short chain fatty acid (SCFA) stress in the large intestine. The research carried out in this doctoral dissertation focuses on understanding how EHEC senses chemical cues from the host's innate immune responses and how this knowledge can be exploited to develop effective antimicrobial strategies. Our findings successfully demonstrate that a novel antimicrobial peptide ameliorates infection in a mouse model of infection by enhancing acid-induced pathogen killing during gastric passage, and that the DNAbinding protein, Dps, plays a significant role in protecting EHEC against peptide killing. Moreover, this research successfully shows that varying concentrations of SCFAs result in differential modulation of EHEC virulence - a finding that contributes to our understanding of the role of diet and commensal flora in host susceptibility to infection. Together the findings of this research demonstrate how the selected innate host defences throughout the human GI tract can be exploited and/or manipulated to effectively prevent infection by the human pathogen EHEC.
\end{abstract}




\section{Acknowledgements}

First and foremost, I would like to express my sincere gratitude to my supervisor, Dr. Debora Barnett Foster, for this opportunity and for her constant mentorship and support throughout my graduate career. I am truly grateful for the ways in which you have challenged me, the opportunities that have been made possible through you, and for the countless hours of advice and motivational speeches that you have given me. I am moving forward as a confident, independent researcher as a result of this incredible journey that I have shared with you.

I am grateful to the members of my supervisory committee, Dr. Costin Antonescu and Dr. Martina Hausner, for their continuous support and invaluable guidance towards my research project. I would also like to thank my lab mom, Kathene Johnson-Henry, for her unconditional support and inspirational advice. Your friendship and confidence in me have been sincerely appreciated.

To the friends that I have made throughout this journey, Camilo Garay, Christopher Choy, Shannon Ho, Hannah Tollman, Crystal Gadishaw-Lue, Aju-Sue Francis, Victoria Hipolito, Stephen Bautista, Monica Dayam, Zechariah Martin, Golam Islam, Krishna Chintaluri, Michele Kacori, Alyssa Banaag, Miriam de Jong, and countless others, I am truly grateful for the many memories that we have shared and for the constant motivation throughout the highs and lows of this academic journey.

Finally, I would like to thank my family - my parents, my sister Candace Lackraj, and my fiancée Shawn Kanhai - for their never-ending support, love, and encouragement throughout the many years of education. Your unwavering belief in me has kept me motivated throughout the long days, endless nights, and trying times. I would not be where I am today without you. 


\section{Table of Contents}

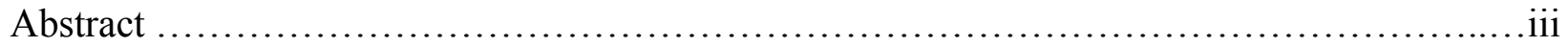

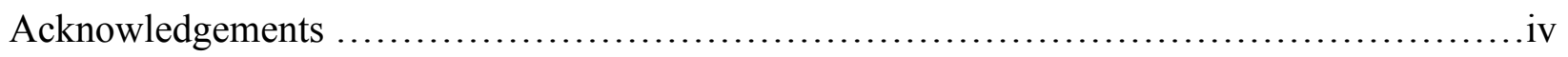

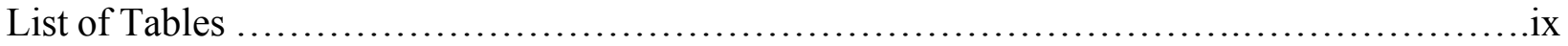

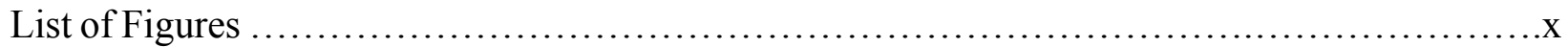

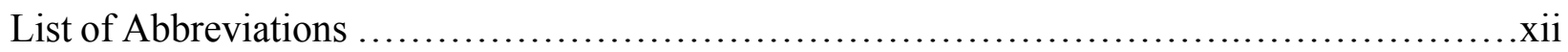

CHAPTER 1: INTRODUCTION - ENTEROHEMORRHAGIC ESCHERICHIA COLI ..........1

1.1 Symptoms and Infection Outcomes .......................................

1.2 Transmission of EHEC and Recent Outbreaks ................................

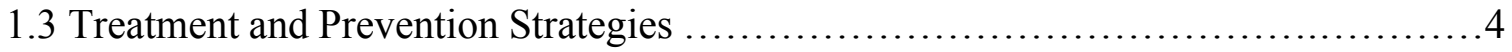

1.3.1 Current Treatment and Prevention Options ............................4

1.3.2 Latest Research on EHEC Treatment and Prevention .......................5

1.4 Interplay Between Human Microbiota and EHEC ................................

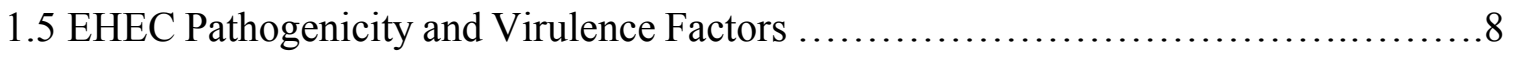

1.6 Virulence Gene Regulation .............................................

1.7 Impact of Human Gut Chemical Signals on EHEC Virulence and Pathogenicity .......14

1.8 Rationale and Thesis Goals ...................................................

CHAPTER 2: EXPLOITING EHEC O157:H7'S RESPONSE TO ACID STRESS AS A NOVEL

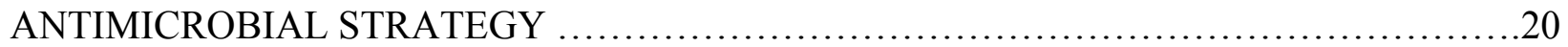

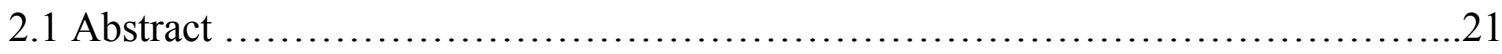

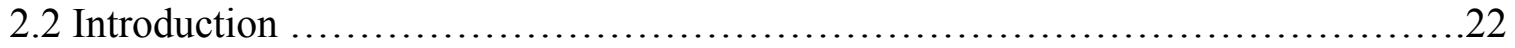

2.2.1 Acute Acid Stress and DNA Damage .............................22

2.2.1.1 Acid Stress as a Defense Mechanism ..........................22

2.2.1.2 Acid Resistance Mechanisms ...............................23

2.2.1.3 DNA Damage and DNA Repair Mechanisms ....................25

2.2.2 Antimicrobial Peptide wrwycr .....................................27

2.2.2.1 Mode of Action ...........................................27

2.2.2.2. Peptide wrwycr as an Antimicrobial ...........................29

2.2.3 Citrobacter rodentium as a Model of EHEC Infection ....................32 
2.2.3.2 Murine Infection Cycle and Infection Outcomes ...................33

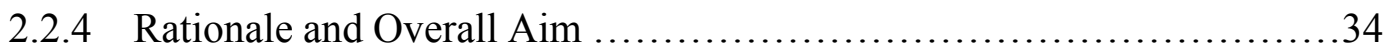

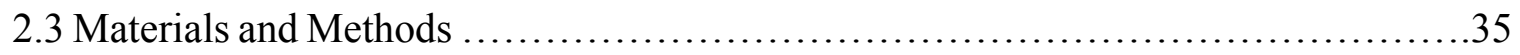

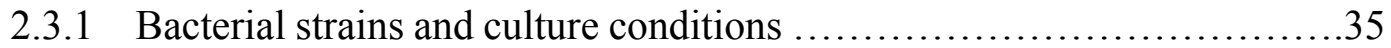

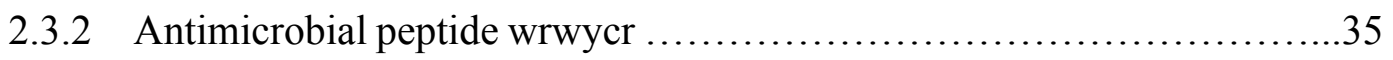

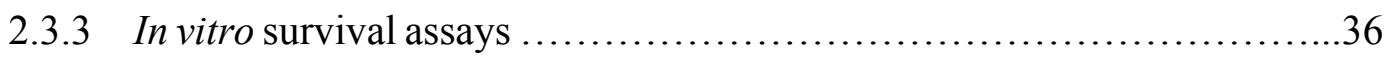

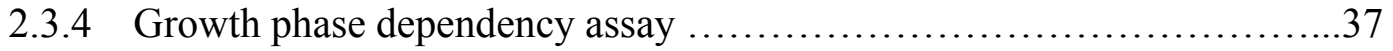

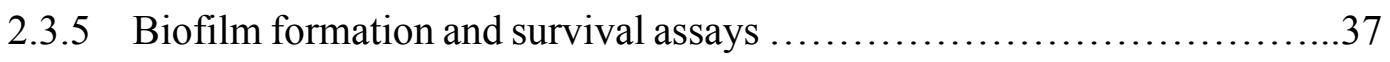

2.3.6 Growth recovery after peptide and peptide-acid treatment ...............38

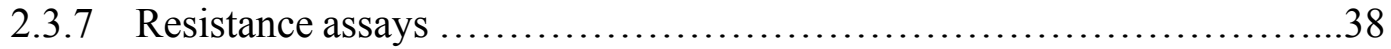

2.3.8 Mouse infection model .................................................39

2.3.9 Histological analysis of colon samples ................................ 40

2.3.10 qPCR analysis of $C$. rodentium infection ..............................40

2.3.11 qPCR analysis of alterations in stool microbial communities .............41

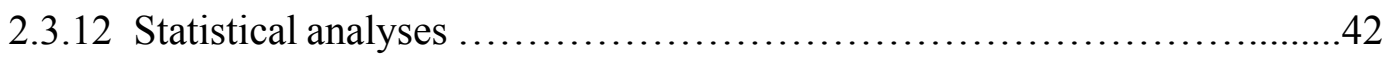

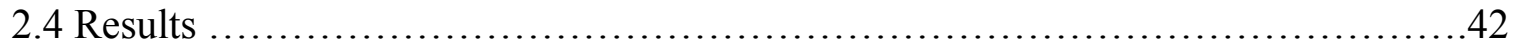

2.4.1 Acute acid stress or peptide treatment alone causes moderate killing of C. rodentium ....................................................... 42

2.4.2 Peptide pre-treatment enhances acid-induced killing of C. rodentium .......45

2.4.3 Peptide treatment does not impact survival of $C$. rodentium biofilms ......46

2.4.4 Growth recovery of peptide- and peptide-acid- treated C. rodentium ......47

2.4.5 Prior exposure of $C$. rodentium to peptide treatment does not induce

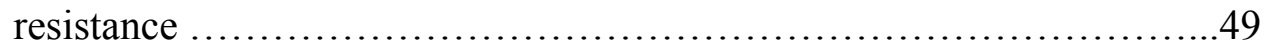

2.4.6 Safety assessment of peptide administration in vivo .....................50

2.4.7 Peptide pre-treatment of C. rodentium ameliorates infection and reduces infection outcomes in mouse infection model ............................51

2.4.8 Peptide pre-treatment reduces $C$. rodentium-induced alterations of the gut

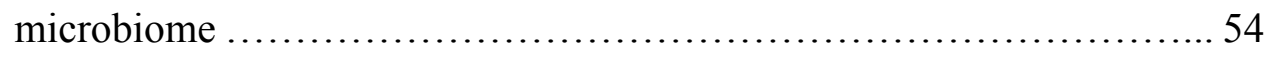

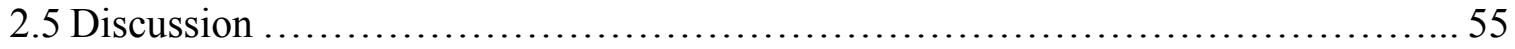

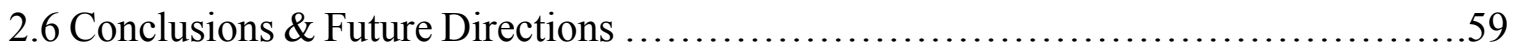




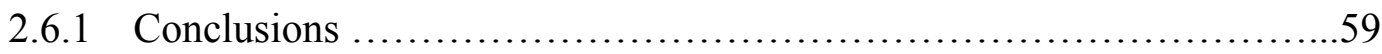

2.6.2 Future Directions \& Significance ...................................61

CHAPTER 3: EXAMINING THE ROLE OF DNA-BINDING PROTEIN, DPS, IN EHEC'S
RESPONSE TO PEPTIDE TREATMENT $\ldots \ldots \ldots \ldots \ldots \ldots \ldots \ldots \ldots \ldots \ldots \ldots \ldots \ldots \ldots \ldots \ldots \ldots \ldots \ldots \ldots \ldots \ldots \ldots \ldots \ldots \ldots \ldots \ldots \ldots$

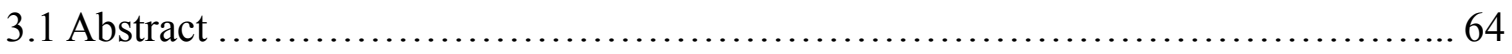

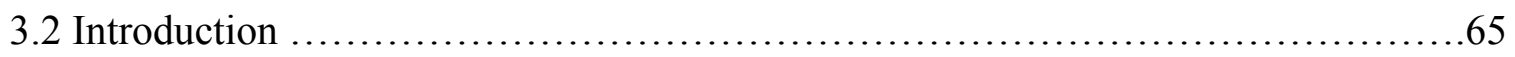

3.2.1 Bactericidal Antibiotics ..........................................65

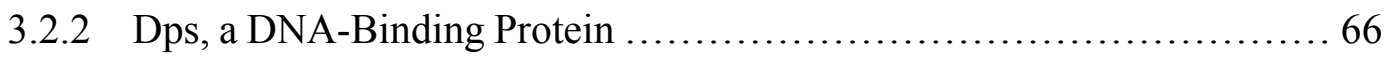

3.2.2.1 Dps as a Protective and Regulatory Protein ......................66

3.2.2.2 DNA-Binding as a Protection Mechanism .........................67

3.2.2.3 Ferroxidase Activity as a Protection Mechanism ....................68

3.2.3 Rationale and Overall Aim ........................................... 69

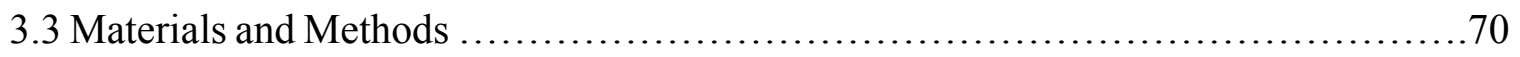

3.3.1 Bacterial strains and culture conditions ............................ 70

3.3.2 Complementation of the $d p s:: n p t I$ mutant .............................70

3.3.3 Antimicrobial peptide wrwycr .................................... 71

3.3.4 Bactericidal versus bacteriostatic antibiotic assay .........................71

3.3.5 Survival assays ..................................................... 71

3.3.6 Flow cytometric analysis of hydroxyl radical formation .................72

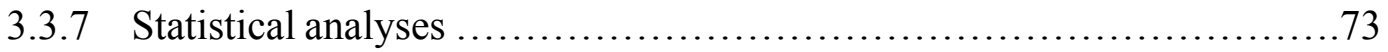

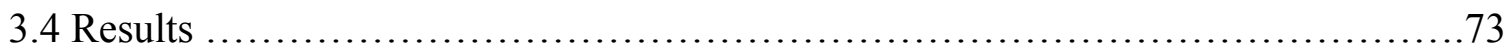

3.4.1 Complementation of the dps::nptI mutant ............................73

3.4.2 Peptide wrwycr behaves as a bactericidal antibiotic ......................74

3.4.3 Disruption of $d p s$ makes EHEC hypersensitive to acid, peptide, and peptide-acid treatment ............................................. 75

3.4.4 Hydroxyl radical formation is increased following peptide treatment of

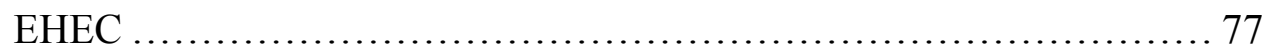

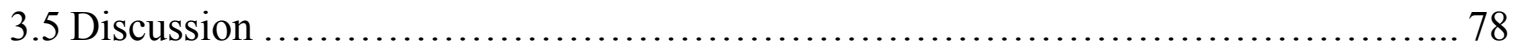

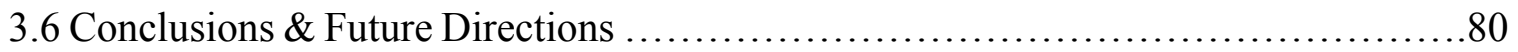

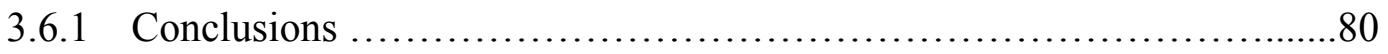


CHAPTER 4: MODULATION OF EHEC O157:H7 VIRULENCE BY SHORT CHAIN

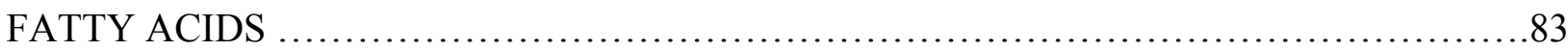

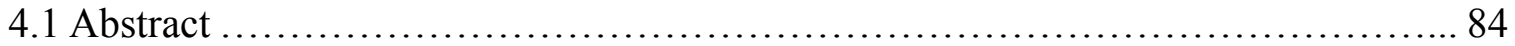

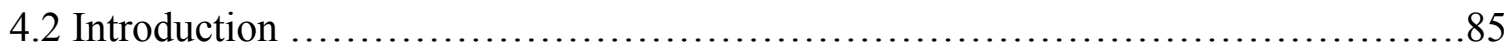

4.2.1 Short Chain Fatty Acids and Colonic Health ...........................85

4.2.2 Short Chain Fatty Acids and Virulence Gene Expression ................89

4.2.3 Rationale and Overall Aim ...........................................91

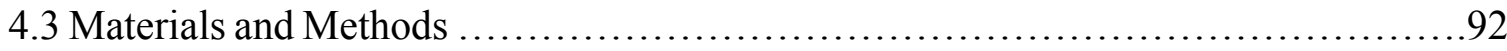

4.3.1 Bacterial strains and culture conditions ............................92

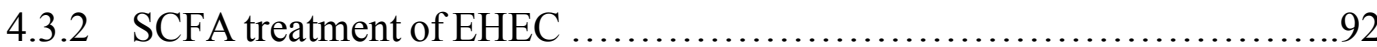

4.3.3 Quantitative real-time PCR analysis of gene expression ...............93

4.3.4 Immunoblot analysis of FliC expression ............................94

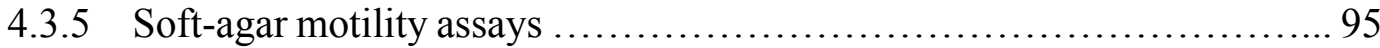

4.3.6 Statistical analysis ........................................... 95

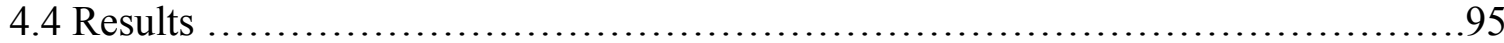

4.4.1 Transcriptome analysis of SCFA-treated EHEC ......................95

4.4.2 FliC protein expression is upregulated in response to 30mM SCFA .......98

4.4.3 EHEC motility is modulated in response to $30 \mathrm{mM}$ and $172 \mathrm{mM}$ SCFA treatment ..........................................................

4.4.4 Impact of individual SCFAs on flagella expression of EHEC ............102

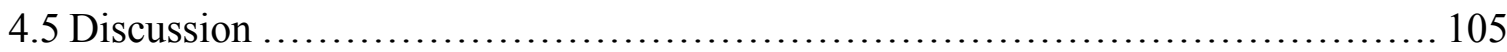

4.6 Conclusions \& Future Directions ........................................ 108

4.6.1 Conclusions ...................................................... 108

4.6.2 Future Directions \& Significance ................................. 110

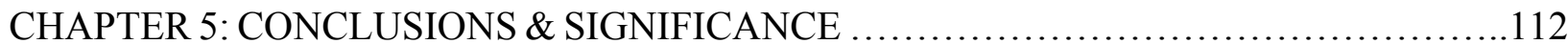

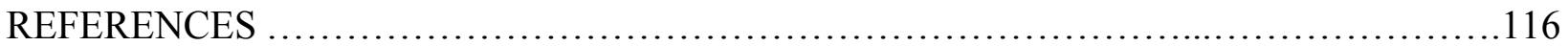




\section{List of Tables}

Table 2.1: Primer sequences used for quantitative PCR of microbial communities............. 41

Table 2.2: Peptide pre-treatment prior to orogastric gavage prevents infection in mice infected with $C$. rodentium compared to uninfected mice in a dose-dependent manner......... 54

Table 3.1: Bacterial strains and plasmids used in study............................... 70

Table 4.1: Primer sequences used for quantitative real-time PCR........................ 94

Table 4.2: Flagellar genes as determined by DNA microarray $\ldots \ldots \ldots \ldots \ldots \ldots \ldots \ldots \ldots \ldots \ldots$ 


\section{List of Figures}

Figure 1.1: Transmission of EHEC ..................................................... 4

Figure 1.2: Overview of EHEC pathogenic mechanism.................................. 9

Figure 1.3: Locus of enterocyte effacement pathogenicity island of EHEC O157:H7 .......... 11

Figure 1.4: Mechanism of Shiga toxin-mediated cell death................................ 13

Figure 2.1: Acid resistance systems in $E$. coli .......................................... 25

Figure 2.2: Catalytic events mediated by Int during site-specific recombination............... 27

Figure 2.3: Peptide wrwycr mode of action............................................. 29

Figure 2.4: Survival of $C$. rodentium following acute acid stress............................ 43

Figure 2.5: Survival of $C$. rodentium following peptide treatment.......................... 44

Figure 2.6: Sensitivity of differential growth phases to peptide treatment of $C$. rodentium...... 45

Figure 2.7: Survival of $C$. rodentium following peptide-acid treatment in vitro.................. 46

Figure 2.8: Survival of established C. rodentium biofilms following peptide treatment.......... 47

Figure 2.9: Growth recovery of $C$. rodentium in vitro................................... 48

Figure 2.10: Resistance of pre-exposed C. rodentium to fresh peptide........................ 49

Figure 2.11: Safety testing of peptide administration in vivo............................... 50

Figure 2.12: In vivo testing of peptide pre-treatment on C. rodentium infection................. 52

Figure 2.13: qPCR analysis of fecal microbial composition of treated mice $10 \mathrm{~d}$ post-infection. 55

Figure 2.14: Model of the antimicrobial peptide wrwycr as a prevention strategy for EHEC infection......................................................... 60

Figure 3.1: Bactericidal antibiotics mode of action............................................. 66

Figure 3.2: Cloning of $d p s \mathrm{ORF}+$ promoter into vector plasmid pUC18 $\ldots \ldots \ldots \ldots \ldots \ldots \ldots \ldots . \ldots \ldots$

Figure 3.3: Comparison of peptide wrwycr to bactericidal and bacteriostatic antibiotics........ 75 
Figure 3.4: Survival of EHEC following acid, peptide, or peptide-acid treatment.............. 77

Figure 3.5: Hydroxyl radical formation in peptide-treated EHEC .......................... 78

Figure 4.1: Chemical structure of SCFAs........................................ 85

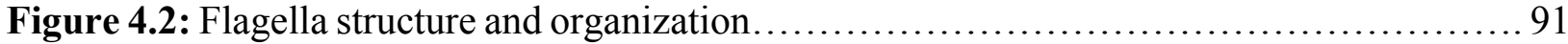

Figure 4.3: Gene expression analysis following SCFA mix treatment of EHEC O157:H7 ...... 98

Figure 4.4: Immunoblot analysis of FliC $(\mathrm{H} 7)$ protein expression following SCFA mix treatment of EHEC O157:H7 .................................................. 99

Figure 4.5: Immunofluorescence analysis of flagella expression following SCFA mix treatment of EHEC O157:H7 ................................................. 100

Figure 4.6: Soft-agar motility analysis following SCFA mix treatment of EHEC O157:H7 ... 101

Figure 4.7: Immunoblot analysis of FliC (H7) protein expression following individual SCFA treatment of EHEC O157:H7 ........................................ 103

Figure 4.8: Immunoblot analysis of FliC (H7) protein expression following a concentration gradient of individual SCFA treatment of EHEC O157:H7 ................... 105

Figure 4.9: Model of the differential modulation of flagella expression by varying concentrations of short chain fatty acids. 109 


\section{List of Abbreviations}

\begin{tabular}{|c|c|}
\hline $\mathrm{A} / \mathrm{E}$ & attaching and effacing \\
\hline AI & autoinducer \\
\hline $\mathrm{Ap}^{\mathrm{R}}$ & ampicillin resistance cassette \\
\hline ANOVA & analysis of variance \\
\hline AR & acid resistance systems \\
\hline CAMPs & cationic antimicrobial peptides \\
\hline cDNA & complimentary DNA \\
\hline $\mathrm{CFU} / \mathrm{mL}$ & colony forming units per milliliter \\
\hline CPPs & cell-penetrating class of peptides \\
\hline C. rodentium & Citrobacter rodentium \\
\hline DAPI & 4',6-diamidino-2-phenylindole \\
\hline DMSO & dimethyl sulphoxide \\
\hline DNA & deoxyribonucleic acid \\
\hline Dps & DNA-binding protein from starved cells \\
\hline EA & ethanolamine \\
\hline ECL & enhanced chemiluminescence \\
\hline EHEC & enterohemorrhagic Escherichia coli \\
\hline Gb3 & globotriaosylceramide-3 \\
\hline GI & gastrointestinal \\
\hline $\mathrm{HCl}$ & hydrochloric acid \\
\hline HJ & Holliday junction \\
\hline $\mathrm{H}-\mathrm{NS}$ & heat-stable nucleoid structuring global regulator \\
\hline
\end{tabular}




\begin{tabular}{|c|c|}
\hline HPF & hydroxyphenyl fluorescein \\
\hline $\mathrm{hr}$ & hours \\
\hline hROS & highly reactive oxygen species \\
\hline HUS & hemolytic uremic syndrome \\
\hline IHF & integration host factor \\
\hline $\mathrm{Km}^{\mathrm{R}}$ & kanamycin resistance cassette \\
\hline LB & Luria Bertani \\
\hline LEE & Locus of Enterocyte Effacement \\
\hline Ler & Lee encoded regulator \\
\hline LPS & lipopolysaccharide \\
\hline MHB & Mueller Hinton Broth \\
\hline $\min$ & minutes \\
\hline MOPS & morpholinepropanesulfonic acid \\
\hline OD600 & optical density 600 \\
\hline PBS & phosphate-buffered saline \\
\hline qPCR & quantitative polymerase chain reaction \\
\hline qRT-PCR & quantitative real-time polymerase chain reaction \\
\hline rRNA & ribosomal RNA \\
\hline SCFAs & short chain fatty acids \\
\hline SDS & sodium dodecyl sulfate \\
\hline SEMs & standard error of the means \\
\hline STEC & shiga-like toxin-producing $E$. coli \\
\hline
\end{tabular}


TBST tris-buffered saline Tween

Tir Translocated Intimin Receptor

tRNA transfer RNA

UT untreated

UV ultraviolet 


\section{Chapter 1: Introduction}

\section{Enterohemorrhagic Escherichia coli}




\subsection{Symptoms and Infection Outcomes}

Enterohemorrhagic Escherichia coli (EHEC) are a class of pathogenic E. coli resulting in food-borne illness that is typically associated with symptoms such as abdominal cramps, diarrhea, hemorrhagic colitis, and may progress to the fatal systemic sequelae known as hemolytic uremic syndrome (HUS) (Karmali et al., 1983; Karmali, 1989; Nguyen and Sperandio, 2012; Rahal et al., 2012; Saxena et al., 2015). HUS is seen in about 16\% of patients infected with EHEC O157:H7, and is a major cause of renal failure in children and mortality in adults (Ho et al., 2013; Saxena et al., 2015).

While the vast majority of E. coli are commensal bacteria typically found in the lower gastrointestinal (GI) tract of mammals (Saxena et al., 2015), a small number of serotypes have been linked to human disease, with the most prevalent serotype associated with human outbreaks being EHEC O157:H7 (Horne et al., 2002; Karmali et al., 2010; Nguyen and Sperandio, 2012). These pathogenic serotypes of $E$. coli are often differentiated from the normal commensal strains by the presence of a number of virulence factors and by distinctive infectious strategies (Horne et al., 2002). EHEC O157:H7 was first identified in 1983 during an outbreak of food-borne related illness caused by undercooked ground hamburger meat at a fast-food chain in the United States (Ho et al., 2013; Riley et al., 1983). According to the Center for Disease Control and Prevention (2008, 2015), there are approximately 95,000 cases of EHEC O157:H7 associated illnesses, 2000 hospitalizations, and 50-60 deaths each year in the United States alone (Ho et al., 2013; Saxena et al., 2015; Serna and Boedeker, 2008). The high number of infections can be attributed to EHEC's low infectious dose, which is less than 100 cells, and high transmissibility (Haas et al., 2000; Ho et al., 2013; Saxena et al., 2015). The disease incubation period for EHEC ranges between 1 to 16 days, with most infections displaying symptoms between 3 to 4 days. Other important factors 
associated with disease outcome include age, general health, immune status, and dose of ingested bacteria (Ho et al., 2013; Saxena et al., 2015).

\subsection{Transmission of EHEC and Recent Outbreaks}

Cattle are the primary reservoir of EHEC, while other ruminants such as sheep, deer, and goat may also act as reservoirs (Gyles, 2007; McNeilly et al., 2010; Persad and LeJeune, 2014; Saxena et al., 2015). Although cattle colonized with EHEC shed the bacterium at levels as high as $1.1 \times 10^{5} \mathrm{CFU} / \mathrm{g}$ feces for up to 10 weeks, the animals remain asymptomatic since they lack the vascular expression of globotriaosylceramide-3 (Gb3) receptors, the receptor bound by Shiga toxin (Fegan et al., 2004; Widiasih et al., 2004). As a result, Shiga toxin is unable to be endocytosed and transported to other organs, thereby not causing adverse effects in ruminants ((Nguyen and Sperandio, 2012; Persad and LeJeune, 2014; Pruimboom-Brees et al., 2000).

Humans can acquire EHEC infection either through direct or indirect contact with animal carriers, their feces, contaminated soil or water, or through the digestion of contaminated animal products or contaminated fruits and vegetables (Figure 1.1), leading to its high level of transmissibility (Saxena et al., 2015). Human infections with EHEC occur worldwide, and have been associated with eating undercooked or unpasteurized animal products, unpasteurized milk and cheese, alfalfa or radish sprouts, lettuce, spinach, and other contaminated vegetables (Center for Food Security and Public Health, 2009). 


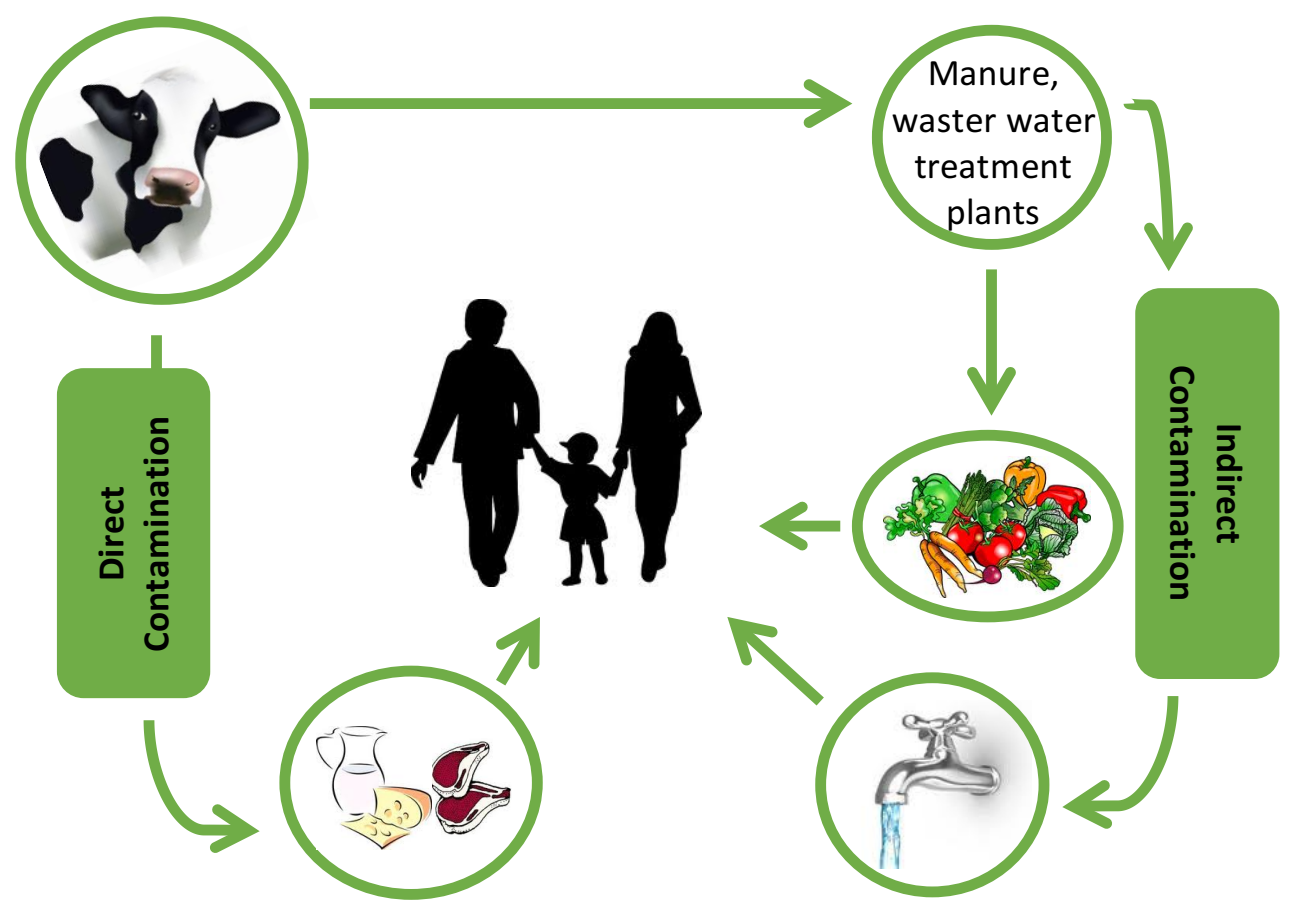

Figure 1.1: Transmission of EHEC. EHEC can be transmitted directly through contaminated animal products or indirectly through contaminated soil, water, fruits, or vegetables.

\subsection{Treatment and Prevention Strategies}

\subsubsection{Current Treatment and Prevention Options}

Currently, patients infected with EHEC are treated mainly with supportive and palliative care consisting of fluid resuscitation, peritoneal dialysis, and plasma exchange (Goldwater and Bettelheim, 2012; Ho et al., 2013; Nguyen and Sperandio, 2012; Saxena et al., 2015). The use of antibiotics, which is the traditional treatment for bacterial infection, is not recommended for treatment of EHEC since there has been much controversy surrounding the efficacy of its use. Antibiotics have been shown to induce expression of Shiga toxin-encoding genes through induction of the SOS response, thereby increasing production of the lethal toxin (Mellies and Lorenzen, 2014; Nguyen and Sperandio, 2012). This, in turn, promotes lysis of the bacterial cell envelope, and as a result, releasing higher amounts of Shiga toxin into the host system and 
increasing the risk of progression to HUS (Ho et al., 2013; Karch et al., 1999; Matsushiro et al., 1999; Mellies and Lorenzen, 2014; Nguyen and Sperandio, 2012; Obrig, 2010; Wong et al., 2012). In an attempt to target the pathogen prior to reaching its human host, cattle vaccines that inhibit colonization of the recto-anal junction have been developed using purified antigens (EspA, Intimin, and Tir) which are important for colonization of ruminant intestines. These vaccines block the attachment process at multiple stages of EHEC colonization and ultimately reduce bacterial shedding by cattle into the environment by over 99\% (Bretschneider et al., 2007; Dean-Nystrom et al., 1998; Cox et al., 2014; McNeilly et al., 2010; Naylor et al., 2007). While the use of cattle vaccination has the potential to drastically reduce the incidence of human infection, in conditions of natural exposure, the cost-benefit of mass vaccination is largely dependent on how prevalently the vaccine is used to control EHEC transmission throughout the production system. Specifically, although there is full regulatory approval for its use in Canada, until there is economic advantage, the widespread use of this vaccination by cattle farmers will remain low (Cox et al., 2014; Smith, 2014).

\subsubsection{Latest Research on EHEC Treatment and Prevention}

In recent years, there has been increased research into the development of new treatment options for EHEC infection. In vitro studies have reported the benefits of probiotics in preventing colonization or spread of EHEC by building up the commensal microflora so as to outcompete the incoming pathogen (Sherman et al., 2005). The use of zinc-based salts has also recently been shown to be effective against EHEC infection outcomes by reducing Shiga toxin levels and pathogen-induced intestinal tissue damage (Dolgin, 2011). Similarly, the novel use of the divalent cation manganese $\left(\mathrm{Mn}^{2+}\right)$ has been implicated in managing human disease by blocking endosome- 
to-golgi trafficking of Shiga toxin in vitro, thereby preventing the spread of Shiga toxin to other organs (Ho et al., 2013; Mukhopadhyay and Linstedt, 2012).

The use of antibody treatment to limit EHEC-induced infection outcomes has been a key target for novel treatment strategies. Thallion Pharmaceuticals produced a dual antibody product known as Shigamabs, which is administered to patients at the onset of EHEC-induced infection symptoms prior to manifestation of HUS (Dolgin, 2011). Shigamabs is a dual antibody system directed at sequestering and neutralizing the two types of Shiga toxin secreted by EHEC, thereby preventing progression of disease to HUS (Dolgin, 2011; Ho et al., 2013; Islam and Stimson, 1990). In contrast, Soliris, otherwise known as Eculizumab, is administered to patients after progression of disease to HUS, and works by reducing inflammation through inhibition of the immune response activated by Shiga toxin (Dolgin, 2011). Specifically, Soliris is a monoclonal antibody targeted against the immune complement protein $\mathrm{C} 5$. It suppresses the immune response by preventing cleavage of C5 and downstream activation of the hemolytic cascade (Dolgin, 2011; Ho et al., 2013; Lapeyraque et al., 2011; Laursen, 2011). The use of this treatment was shown to successfully reverse HUS-associated kidney damage and neurological symptoms seen in 3 children infected with EHEC (Dolgin, 2011).

Our laboratory has recently identified a novel antimicrobial peptide wrwycr that enhances pathogen killing when the pathogen is pre-treated with peptide and then exposed to acute acid stress similar to gastric passage (Lino et al., 2011). This study demonstrates high potential as a prevention strategy against EHEC infection and provides persuasive evidence for in vivo studies (Lino et al., 2011). 


\subsection{Interplay Between Human Microbiota and EHEC}

The human microbiota consists of 10-100 trillion microbial cells that live in a symbiotic manner in the human body, with the vast majority of these microbial cells residing in the GI tract and belonging to many bacterial phyla, primarily Firmicutes and Bacteriodetes (Pifer and Sperandio, 2014; Qin et al., 2010; Tremaroli and Bäckhed, 2012; Ursell et al., 2012). The initial microbiota of an individual is a reflection of what is maternally transferred at birth. This is subsequently grown and dynamically changed in response to diet, life-style, disease, and treatment throughout a person's life (Murgas Torrazza and Neu, 2011; Nicholson et al., 2012; Ravel et al., 2011). While each person has a highly distinct and unique microbiota, there is a conserved set of bacteria that are shared amongst individuals and referred to as the core gut microbiota. It is speculated that this core set of microbial species may be essential for correct functioning of the gut (Pifer and Sperandio, 2014; Qin et al., 2010; Tremaroli and Bäckhed, 2012; Turnbaugh et al., 2009). The GI microbiota serves important roles in human nutrition, physiology, development, immunity, and behaviour. The role of the gut microbiota in host metabolism can be dietindependent or diet-dependent. An example of diet-independent metabolism is the response of the host in producing proteins to mediate the immune response to microbiota pro-inflammatory molecules such as lipopolysaccharide (LPS) and peptidoglycan. In contrast, diet-dependent metabolism may involve the breakdown of cholesterol and polysaccharides to produce primary and secondary bile acids and short-chain fatty acids respectively, which can lead to protection against heart disease or modulation of inflammation (Tremaroli and Bäckhed, 2012). Disruption of the balance between the host and the microbiota, termed dysbiosis, can be caused by changes in diet, administration of antibiotic treatment, and disease, and may result in changes to the intestinal metabolic profiles and invasion by opportunistic pathogens (Pifer and Sperandio, 2014). 
Since EHEC, like other enteric pathogens, colonizes the distal colon, it must interact and compete with the gut microbiota in order to successfully colonize its host. The interaction between the host and microbiota typically results in a multidimensional gradient of metabolites within the GI tract, creating distinct environmental conditions that can be utilized by EHEC as a signal for regulation of its virulence systems in order to maximize its infectious ability (Pifer and Sperandio, 2014). Additionally, EHEC also regulates virulence gene expression through the use of quorumsensing signalling. While EHEC uses multiple quorum sensing systems such as the luxS/autoinducer (AI) 2, AI3/epinephrine/norepinephrine, indole, and the luxR homolog SdiA to achieve intercellular communication, many of the commensal microbiota also produce both AI2 and AI3. It has been hypothesized that this mode of communication may be utilized by EHEC to signal the microbiota of its presence in the large intestine and also as a means to modulate virulence gene expression in response to the presence of the commensal microbiota (Hernandez-Doria and Sperandio, 2013; Pifer and Sperandio, 2014; Sperandio et al., 1999, 2003).

Whether through the production of chemical gradients or quorum sensing, it is clear that the gut microbiota plays a vital role in the ability of EHEC to successfully colonize and infect the host.

\subsection{EHEC Pathogenicity and Virulence Factors}

EHEC colonizes the human large intestine at Peyer's patches in the distal ileum (Chong et al., 2007; Croxen and Finlay, 2009; Ståhl and Karpman, 2014). Infection is characterized by adhesion to host epithelial cells, subversion of host cell signalling pathways and processes, evasion of host immune responses and efficient colonization which ultimately results in disease (Figure 1.2) (Bhavsar et al., 2007; Croxen and Finlay, 2009). Initial attachment of EHEC to host epithelial cells is mediated by appendages such as fimbriae, pili, and flagella (Croxen and Finlay, 2009; 
Erdem et al., 2007; Rendón et al., 2007). A more intimate attachment is then developed through the formation of attaching and effacing (A/E) lesions, which are a hallmark feature of enteric pathogens. The $\mathrm{A} / \mathrm{E}$ lesions are characterized by destruction of microvilli on the epithelial cells, and accumulation of polymerized actin beneath the surface of bacterial adherence, resulting in a raised pedestal-like structure and a more intimate attachment with individual bacteria (Afset et al., 2004; Bueris et al., 2007; Estrada-Garcia et al., 2009; Kaper et al., 2004; Nataro and Kaper, 1998;

Nguyen and Sperandio, 2012; Saxena et al., 2015).

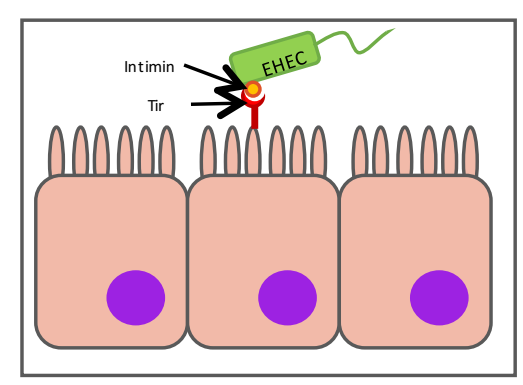

1. Adherence to epithelial cells

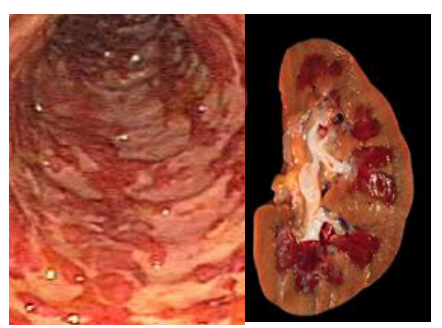

6. Systemic disease and complications

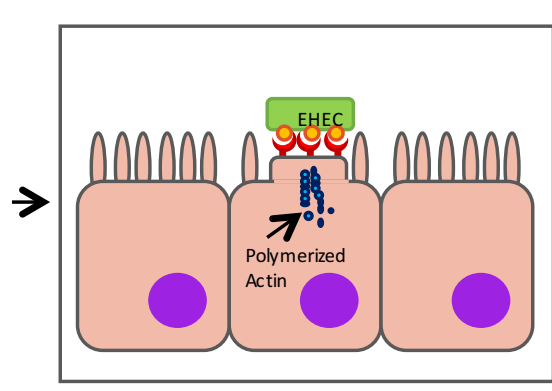

2. Formation of $A / E$ lesions

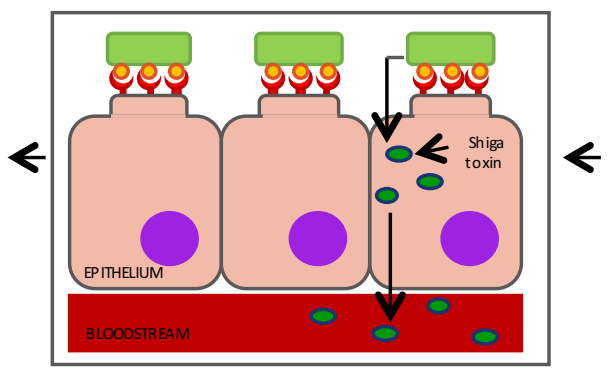

5. Production and secretion of toxins

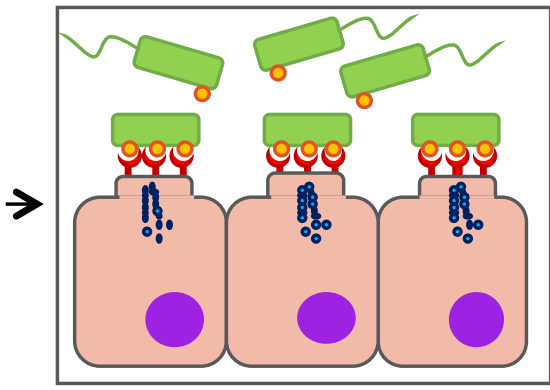

3. Colonization of the gut $\checkmark$

4. Manipulation of host cell intracellular signalling pathways

Figure 1.2: Overview of EHEC pathogenic mechanism. EHEC adherence to epithelial cells is characterized by binding of the cellular membrane protein intimin to the translocated receptor Tir, followed by formation of a pedestal-like structure caused by actin polymerization beneath the site of attachment. This is followed by manipulation of host cell intracellular signalling pathways, production and secretion of the characteristic Shiga toxin into the bloodstream, and downstream complications and disease. 
In order to successfully hijack host cell signalling pathways, EHEC utilizes a molecular syringe, known as the Type 3 Secretion System (T3SS), to inject approximately 50 distinct bacterial-encoded effector proteins directly into the host cell cytoplasm (Mellies and Lorenzen, 2014; Tobe et al., 2006). The T3SS is comprised of over 16 proteins that form a channel leading from the bacterial cytoplasm, across the inner and outer membranes, into the cytoplasm of the host cell (Daniell et al., 2001; Ebel et al., 1998; Horne et al., 2002; Knutton et al., 1989). One of the first secreted proteins to be injected into the host cell via the T3SS is the Translocated Intimin Receptor (Tir), which is redirected to the host cytoplasmic membrane with its central domain exposed to the extracellular environment. The central domain of Tir then interacts with the EHEC surface protein intimin to form the tight attachment of the $\mathrm{A} / \mathrm{E}$ lesion, thereby playing a critical role in EHEC colonization (Deibel et al., 1998; Horne et al., 2002; Kenny et al., 1997b; Mellies and Lorenzen, 2014). Additional T3SS effector proteins include EspA, EspB, EspD, EspF, SepL, EscF, which mediate numerous effects including host cytoskeleton rearrangement leading to the formation of an actin pedestal and also suppression of the host immune response (Donnenberg et al., 1993; Franzin and Sircili, 2015; Garmendia et al., 2005; Horne et al., 2002; Ide et al., 2001; Knutton et al., 1989; Sekiya et al., 2001).

The vast majority of effector proteins are encoded on the Locus of Enterocyte Effacement (LEE) pathogenicity island, a $35.6 \mathrm{~Kb}$ island that consists of approximately 41 genes divided into 5 major operons (LEE 1-5) (Figure 1.3) (Elliott et al., 1998; Knutton et al., 1989; Mellies and Lorenzen, 2014; Moon et al., 1983; Nguyen and Sperandio, 2012). The genes present on the LEE pathogenicity island encode many of EHEC's virulence factors including the T3SS regulators, chaperones, and effector proteins secreted by the T3SS. There are also a number of non-LEEencoded effectors that include proteins critical for EHEC colonization such as EspJ, NleB, NleE, 
NleF, and NleH (Echtenkamp et al., 2008; Hemrajani et al., 2008; Kelly et al., 2006; Mellies and Lorenzen, 2014). Another vital non-LEE encoded effector protein is the E. coli secreted protein Flike protein from prophage $\mathrm{U}$, which when secreted into the host cell works with Tir to recruit actin nucleation-promoting factor, Wiskott-Aldrich syndrome protein, and insulin receptor tyrosine kinase substrate $\mathrm{p} 53$, in order to effectively subvert the host cytoskeleton and promote actin polymerization (Campellone et al., 2004; Nguyen and Sperandio, 2012; Weiss et al., 2009).

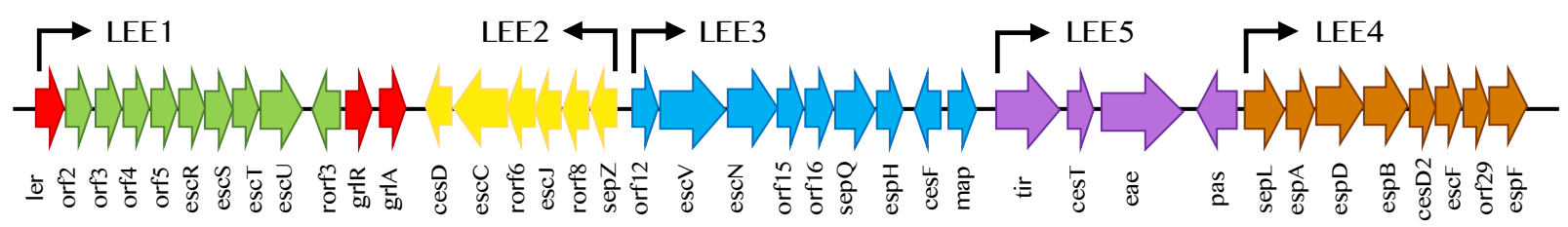

Figure 1.3: Locus of enterocyte effacement pathogenicity island of EHEC O157:H7. Adapted from (Ritchie and Waldor, 2005).

A subtype of EHEC known an Shiga-toxin producing Escherichia coli (STEC), produce a critical virulence factor Shiga toxin, which is strikingly similar to the toxin produced by Shigella dysenteriae type 1 (Obrig, 2010). While STEC strains can produce two different types of Shiga toxin, Stx1 and Stx2, the combination in which they are produced is strain-dependent (Mead and Griffin, 1998). Shiga toxin is comprised of two major subunits, A and B, with the B subunit forming a pentamer that is noncovalently bound to the enzymatically active A subunit (Croxen and Finlay, 2009; Lingwood et al., 1987; O’Brien et al., 1992; Paton and Paton, 1998; Toshima et al., 2007). After Shiga toxin is released into the intestinal lumen and into enterocytes by EHEC, it binds to the Gb3 receptor on endothelial cells, thereby permitting entry of toxin into the bloodstream and circulation to other organs (Croxen and Finlay, 2009; Malyukova et al., 2009; Sandvig, 2001). While many cell types produce the Gb3 receptor and are sensitive to Shiga toxin, the receptors are particularly abundant on renal glomerular endothelium cells in the kidney 
resulting in acute renal failure, thrombocytopenia, and microangiopathic hemolytic anemia which are all characteristic of HUS (Karmali et al., 1983; Nguyen and Sperandio, 2012; PruimboomBrees et al., 2000; Rahal et al., 2012).

When the Shiga toxin B subunit binds to the Gb3 receptor on the cell membrane, more toxin-receptor complexes are aggregated in clathrin-coated pits leading to internalization of Shiga toxin by endocytosis (Figure 1.4) (Kurmanova et al., 2007; Rahal et al., 2012; Ståhl and Karpman, 2014). Once internalized, it is then trafficked through endosomes to the trans golgi network, where it is cleaved into the A and B subunits. The activated subunits are then transported to the endoplasmic reticulum where it is translocated to the host cell cytoplasm. Once in the cytoplasm, the A subunit which is a $28 \mathrm{~S}$ ribosomal RNA (rRNA) $\mathrm{N}$-glycosidase proceeds to disrupt protein synthesis by cleaving an adenine residue from a specific nucleotide of the 28S rRNA component on the $60 \mathrm{~S}$ ribosomal subunit. This prevents tRNA from binding to the $60 \mathrm{~S}$ ribosomal subunit, which prevents peptide elongation and eventually leads to cell death (Croxen and Finlay, 2009; Hofmann, 1993; Jackson, 1990; Rahal et al., 2012; Sandvig and van Deurs, 1996).

Together, the virulence factors utilized by EHEC represent a carefully orchestrated plan to infect intestinal epithelial cells, subvert host cell signalling pathways, and target other organs to cause EHEC-mediated disease outcomes such as progression to HUS. 


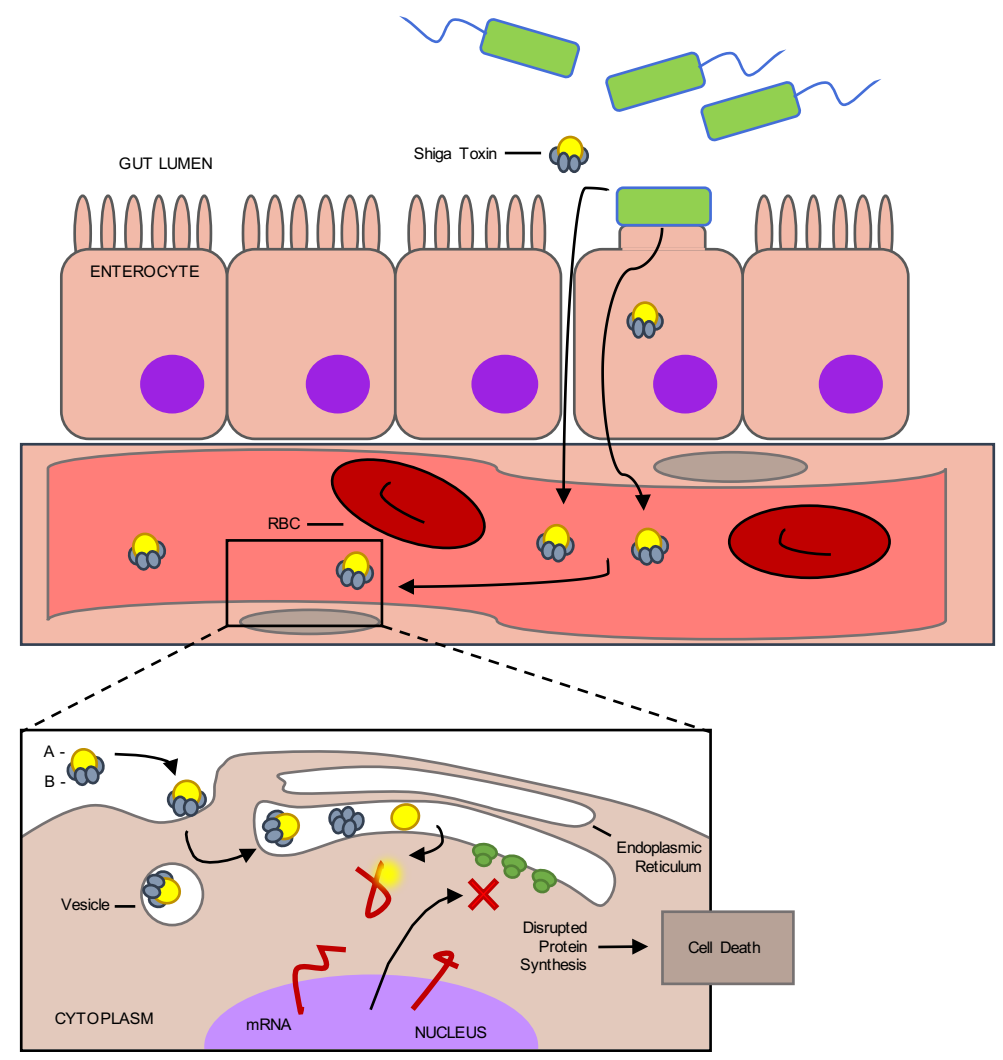

Figure 1.4: Mechanism of Shiga toxin-mediated cell death. Shiga toxin is produced and secreted by EHEC into the host bloodstream where it is internalized by endothelial cells. Once in the endoplasmic reticulum, the A and B subunits separate, allowing the A subunit to bind mRNA thereby disrupting protein synthesis and ultimately leading to cell death. Adapted from http://what-when-how.com/acp-medici ne/infections-due-to-escherichia-coli-and-other-enteric-gram-negative-bacilli-part-1/.

\subsection{Virulence Gene Regulation}

Since the LEE pathogenicity island harbors the vast majority of EHEC's virulence factors, expression must be tightly regulated in order to optimize the infectious potential of the pathogen when necessary. LEE gene expression is induced as EHEC transitions from exponential to stationary phase, and is modulated in response to nutrient deprivation and quorum sensing signalling (Sperandio et al., 2003). The first gene in LEE1 encodes the Lee encoded regulator (Ler), which is a transcription factor that acts as the master regulator of the entire LEE island 
(Elliott et al., 1998; Jerse et al., 1990; Kaper et al., 2004; Nguyen and Sperandio, 2012). The 15kDa Ler protein, an H-NS homolog, regulates LEE expression through its action as an anti-silencer, thereby inducing LEE expression (Elliott et al., 2000; Mellies and Lorenzen, 2014). While Ler expression is stimulated in response to distinct environmental cues including temperature, $\mathrm{pH}$, iron, ammonium, calcium, bicarbonate, and quorum sensing (Abe et al., 2002; Beltrametti et al., 1999; Kenny et al., 1997a; Sperandio et al., 1999), it is also under control of numerous regulatory factors including the global regulator of LEE activator, the integration host factor (IHF), and the heat-stable nucleoid-structuring (H-NS) global regulator (Franzin and Sircili, 2015; Friedberg et al., 1999). H-NS acts as a negative regulator to repress ler expression, while IHF works in opposition to H-NS by binding upstream from the ler promoter to disrupt repression of ler by HNS (Franzin and Sircili, 2015; Friedberg et al., 1999).

This tightly coordinated regulation of virulence is critical to ensuring EHEC colonization and infection, and the impact of distinct environmental cues on virulence gene regulation has been the focus of extensive research recently.

\subsection{Impact of Human Gut Chemical Signals on EHEC Virulence and Pathogenicity}

In order for EHEC to successfully colonize the human distal colon, it must first overcome various microenvironmental stresses encountered throughout the GI tract such as mucins and enzymes present in saliva, acid stress in the stomach, bile secretion in the small intestine, and antimicrobial peptides throughout the small and large intestine (Ståhl and Karpman, 2014). In order to successfully infect the host, EHEC must not only overcome these host assaults, but as recent research shows, may also use them as chemical signals to cue virulence expression. 
One of the first major stresses encountered during passage through the human GI tract is acute acid stress in the stomach, ranging in $\mathrm{pH}$ from 2-6 depending on the state of feeding and diet. Exposure to acute acid can be lethal to enteric pathogens due to cytoplasmic acidification and depurination of bacterial DNA. As a result of its ability to compromise bacterial survival, EHEC must survive this stress in order to complete passage through the stomach and reach its site of colonization. Both non-pathogenic and pathogen E. coli have been shown to encode four different acid resistance (AR) systems which provide protection against acute acid exposure as low as $\mathrm{pH} 2$ (Barnett Foster, 2013; Foster, 2004; Lin et al., 1996; Pifer and Sperandio, 2014; Ståhl and Karpman, 2014). Interestingly, EHEC's response to acute acid has also been shown to induce several virulence properties such as enhanced motility and cell adhesion, but has no impact on Shiga toxin expression (Barnett Foster, 2013; House et al., 2009; Ståhl and Karpman, 2014).

Once EHEC has exited the stomach and entered the small intestine, it then faces bile acid secretion from the gall bladder. The presence of bile acids in the small intestine serve several important functions such as absorption of dietary fats and lipid-soluble vitamins, maintaining intestinal barrier function, and signalling of systemic endocrine functions to regulate triglycerides, cholesterol, glucose, and energy homeostasis (Groh et al., 1993; Nicholson et al., 2012; Ridlon et al., 2006; Swann et al., 2011). Additionally, bile has been shown to have a bactericidal role against enteric bacteria through its damaging effects on DNA and lipid membrane integrity. As a result, bile resistance is a vital characteristic for enteric bacteria (Kus et al., 2011; Merritt and Donaldson, 2009). EHEC resistance to bile is achieved through upregulation of a variety of resistance nodulation division efflux systems in addition to altered outer membrane permeability which is achieved through LPS modifications (Barnett Foster, 2013; Kus et al., 2011; Lacroix et al., 1996; Wibbenmeyer et al., 2002). 
Following exposure to bile acids, EHEC is exposed to cationic antimicrobial peptides (CAMPs), produced by paneth cells near the end of the small intestine. These CAMPs are part of the innate immune system and are attracted to the negatively charged outer membrane of the bacteria (Kus et al., 2011). These peptides serve a bactericidal role against incoming pathogens by disrupting membrane integrity through the formation of pores within the membrane, and by targeting intracellular molecules to disrupt function (Kus et al., 2011; Nguyen et al., 2011). Interestingly, previous research has demonstrated that exposure of EHEC to bile salts increases resistance to CAMP exposure, thereby allowing the bacteria to survive passage through this vital innate immune defense strategy (Barnett Foster, 2013; Kus et al., 2011).

After exposure to antimicrobial peptides in the small intestine, EHEC encounters various membrane lipid metabolites including ethanolamine (EA) in the large intestine, a result of the constant turnover of intestinal epithelial cells and microbiota within the GI tract (Garsin, 2012). In addition to serving as a nitrogen source for EHEC through the breakdown of EA, it may also act as an important cue for virulence gene regulation by increasing expression of quorum-sensing regulators QseA, QseC, QseE, and Ler (Barnett Foster, 2013; Mellies and Lorenzen, 2014). Quorum sensing of AI2 and AI3 in turn induce expression of LEE-encoded and non-LEE-encoded virulence factors including flagella and Shiga toxin (Hernandez-Doria and Sperandio, 2013; Sperandio et al., 1999, 2003). In addition to quorum sensing, EHEC also utilizes the host-generated hormones, epinephrine and norepinephrine, as signals to modulate virulence factor expression such as motility and A/E lesion formation (Barnett Foster, 2013; Clarke et al., 2006; Njoroge and Sperandio, 2012). EHEC also senses low oxygen levels in the large intestine and uses it to modulate virulence accordingly. Specifically, while the large intestine is mainly anaerobic, there is a microaerophilic environment adjacent to the intestinal microvilli. EHEC may sense this and 
as a result upregulate EHEC virulence factors that promote colonization of the intestinal epithelium (Barnett Foster, 2013; Schüller and Phillips, 2010)

Once in the small and large intestines, EHEC is exposed to increasing concentrations of short chain fatty acids (SCFAs), primarily composed of acetate, butyrate, and propionate in varying compositions. SCFAs serve an important role in the large intestine by decreasing colonic $\mathrm{pH}$, inhibiting growth of pathogens, stimulating water and sodium uptake, providing energy to colonic epithelial cells, and participating in cholesterol synthesis. The presence of SCFAs at high concentrations has been shown to exhibit toxic effects on bacteria by decreasing cytoplasmic $\mathrm{pH}$, thereby compromising metabolic reactions, and by impacting cellular physiology such as osmotic balance (Sun and O'Riordan, 2013). However, research indicates that EHEC may use SCFAs as an environmental signal of the site of colonization to induce T3SS expression, increase adhesion, increase motility, and facilitate formation of microcolonies (Barnett Foster, 2013; Herold et al., 2009; Nicholson et al., 2012; Pifer and Sperandio, 2014; Samuel et al., 2008; Ståhl and Karpman, 2014).

It is clear that while these various host assaults are designed to protect against incoming pathogens, EHEC may utilize these as environmental signals to modulate its virulence appropriately to overcome them and successfully infect the host.

\subsection{Rationale and Thesis Goals}

With numerous EHEC outbreaks seen worldwide, there is an increased interest in the development of new treatment options, particularly preventative strategies that target $E$. coli before it is able to colonize and infect the host. In order to develop effective therapeutics against any pathogen, it is critical to understand how the pathogen regulates its virulence factors to optimize 
its infectious potential. In the case of EHEC, a foodborne pathogen that infects the lower GI tract, a key aspect in developing novel therapeutics is to gain insight into how EHEC utilizes distinct gastrointestinal microenvironments as cues to modulate its virulence. Once a thorough understanding of this virulence regulation is achieved, the knowledge can be used to identify vulnerabilities in EHEC's pathogenic mechanisms that can be exploited in order to treat or prevent infection.

This research project focuses on two main environmental stresses present within the human GI tract: acute acid stress in the stomach and SCFA stress in the small and large intestines. Since acute acid stress induces high levels of bacterial DNA damage, that can result in cell death if left unrepaired, it represents a significant vulnerability that can be exploited for development of preventative strategies. Previous studies have identified a novel antimicrobial peptide wrwycr that significantly reduces pathogen survival when administered prior to acid stress similar to that encountered during gastric passage (Lino et al., 2011). Since these studies showed promising results for the use of this peptide as a prevention strategy in vitro, this provides compelling evidence to investigate this peptide in vivo using a suitable model of EHEC infection. Secondly, SCFAs have been implicated in modulation of virulence of several enteric pathogens including EHEC (Lawhon et al., 2003; Nakanishi et al., 2009; Tobe et al., 2011). Since SCFAs are present along a concentration gradient throughout the small and large intestines, a thorough understanding of how EHEC responds to differing concentrations of SCFAs is a vital aspect in understanding the mechanisms utilized to increase EHEC's potential to infect its host, and in identifying novel therapeutic targets. The specific research objectives of this thesis are listed below. 
1. Examining the potential of a novel antimicrobial peptide as a preventative agent against EHEC infection using a suitable in vivo model.

2. Understanding the role of DNA binding protein, Dps, in EHEC's response to acid and peptide treatment.

3. Investigating modulation of EHEC virulence in response to varying concentrations of short chain fatty acids.

This thesis is organized into 6 chapters. Chapter 1 provides an overview of EHEC virulence and pathogenicity, while chapters 2,3 , and 4 represent independent research projects reflective of the 3 specific objectives listed above. Each of these chapters includes a more thorough introduction, materials and methods, results, discussion, conclusions, and future directions reflective of the respective study. Chapters 2 and 4 are based on the published manuscripts listed at the beginning of each chapter. Chapter 5 discusses the overall conclusions from the dissertation as a whole, followed by a complete reference list in chapter 6 . 


\section{Chapter 2: Exploiting EHEC 0157:H7's Response to Acid Stress as a Novel Antimicrobial Strategy}

Lackraj, T., Johnson-Henry, K., Sherman, P., Goodman, S., Segall, A., \& Barnett Foster, D. (2016) Novel antimicrobial peptide prevents $C$. rodentium infection in C57BL/6 mice by enhancing acid-induced pathogen killing. Microbiology doi:10.1099/mic.0.000335. 


\subsection{ABSTRACT}

Citrobacter rodentium is a gram-negative, murine-specific enteric pathogen that infects epithelial cells in the colon. It is closely related to the clinically relevant human pathogen, enterohemorrhagic Escherichia coli (EHEC), a leading cause of hemorrhagic colitis and hemolytic uremic syndrome. We have previously reported that a novel antimicrobial peptide, wrwycr, compromises bacterial DNA repair and significantly reduces the survival of acid-stressed EHEC, suggesting an antimicrobial strategy for targeting the survival of ingested EHEC. This study examines the impact of peptide pre-treatment followed by acid stress on survival and infection of the closely related murine pathogen, $C$. rodentium, using both in vitro and in vivo investigations. Peptide pre-treatment of $C$. rodentium significantly and dramatically increases acid stress-induced killing in a peptide-dose- and time-dependent manner. Reduction in survival rates after brief pretreatment with peptide $(25-65 \mu \mathrm{M})$ followed by 1 hour at $\mathrm{pH} 3.5$ range from 6 to 8 log fold, with no detectable bacteria after $65 \mu \mathrm{M}$ peptide pre-treatment. Using a C57BL/6 mouse model of infection, peptide pre-treatment of $C$. rodentium with wrwycr prior to orogastric gavage significantly reduced fecal bacterial counts and $E s p B$ expression to below detectable limits, as well as eliminating evidence of colonic crypt hyperplasia, and all visible signs of illness. Additionally, peptide pre-treament prior to orogastric gavage also reduced $C$. rodentium-induced changes in the gut microbiota. Of particular interest, the in vivo studies also revealed that peptide treatment alone did not induce changes in the gut microbiota, providing evidence that its administration is safe to the host. These findings provide compelling evidence for the role of the peptide wrwycr as a preventative strategy against enteric pathogens through a novel approach to enhance the body's natural defense system of gastric acid to compromise bacterial survival prior to gut colonization. 


\subsection{INTRODUCTION}

\subsubsection{Acute Acid Stress and DNA damage}

\subsubsection{Acid Stress as a Defense Mechanism}

For enteric pathogens, the journey from ingestion to successful colonization of the host intestine represents a series of host assaults meant to act as a barrier to pathogen infection. Among these host assaults is the exposure to acute gastric acid stress in the stomach, which acts as one of the primary lines of defense against incoming pathogens (Lin et al., 1996). The human stomach represents an acidic environment with an average $\mathrm{pH}$ of 2.0 and an emptying time of approximately 2 hours, although this varies depending on the type and amount of food consumed (Richard and Foster, 2004; Smith, 2003). Passage from the stomach into the small intestine provides a less extreme acidic environment, although the presence of fermentation end products produced by the normal intestinal flora result in the presence of weak acids, thereby creating an environment ranging in $\mathrm{pH}$ from 4 to 6 (Lin et al., 1996).

Highly acidic environments such as that encountered in the human stomach results in extreme toxicity that can be detrimental to enteric pathogens due to cytoplasmic acidification which leads to denaturation of macromolecules and disruption of enzymatic function (Calhoun and Kwon, 2011; Jeong et al., 2008; Lindahl and Nyberg, 1972; Smith, 2003). These damaging effects of acute acidity result in bacterial cell death and decrease the potential for successful colonization of the host intestine. Interestingly, research has demonstrated that mice containing a mutation in a gastric proton pump gene, and thereby constitutively hypochlorhydric, had significantly higher numbers of bacteria surviving passage through the stomach resulting in lower infectious doses compared to mice lacking the mutation (Tennant et al., 2008). These results 
confirm that the presence of stomach acid poses a significant antimicrobial environment that acts as a barrier between enteric pathogens and successful infection.

\subsubsection{Acid Resistance Mechanisms}

In order to successfully infect its host, the ability of bacteria to survive the extreme acidity of the stomach is critical. Human enteric pathogens such as Salmonella and E. coli have adapted a robust response to acid stress which includes induction of both physiological and biochemical changes upon exposure to extreme acidic environments (Goodson and Rowbury, 1989; Jeong et al., 2008; Swenson et al., 2012). The ability to induce such mechanisms allow microorganisms to be better equipped to survive acid challenge and cause disease (Lin et al., 1996). The strategies that these pathogens utilize to maintain acid tolerance can be classified into three categories. The first of these involves changes in membrane composition to promote membrane exclusion of proteins (Brown et al., 1997), while the second strategy involves physiological and enzymatic maintenance of pH homeostasis (Audia et al., 2001; Castanie-Cornet et al., 1999). Finally, the third strategy involves prevention and/or repair of damage inflicted on essential cellular macromolecules (Audia et al., 2001; Cheville et al., 1996; Jeong et al., 2008; Raja et al., 1991). Moreover, the induction of acid stress tolerance has also been shown to activate additional adaptive behaviours which include virulence, biofilm formation, chemotaxis, and antibiotic resistance (Swenson et al., 2012).

In addition to these acid tolerance strategies, both non-pathogenic and pathogenic E. coli encode four inducible acid resistance systems (AR1-4) that provide protection in extreme acidic environments as low as pH 2.0-2.5 (Barnett Foster, 2013; Castanie-Cornet et al., 1999; Foster, 2004). The first of these systems AR1, also referred to as the oxidative or glucose-repressed 
system, employs the stationary phase alternative sigma factor RpoS and the cyclic AMP receptor protein and provides an acid tolerance response that allows $E$. coli exposed to sublethal $\mathrm{pH}$ values to survive subsequent exposure to lower pH values (Castanie-Cornet et al., 1999; Foster, 2004). The second and third acid resistance systems, AR2 and AR3, require extracellular glutamate and arginine respectively (Figure 2.1). AR2 is induced during stationary phase or during log-phase growth in acidic minimal media, and decarboxylates glutamate to form the intracellular end product GABA (Castanie-Cornet et al., 1999; Richard and Foster, 2004). Similarly, AR3 decarboxylates arginine to form the intracellular end product agmatine when induced by low $\mathrm{pH}$ under anaerobic conditions in complex media (Castanie-Cornet et al., 1999; Gong et al., 2003; Richard and Foster, 2004). Finally, the final acid resistance system, AR4, was recently described as being lysine-dependent, and similarly to AR2 and AR3 likely involves the inducible lysine decarboxylase (Iyer et al., 2003; Richard and Foster, 2004). Together, these acid resistance systems enable enteric pathogens to resist low $\mathrm{pH}$ exposure, a strategy which can be critical for these microorganisms to survive in acidic foods, food-processing treatments, and passage through the gastro-intestinal tract (Conner and Kotrola, 1995; Lin et al., 1996). 


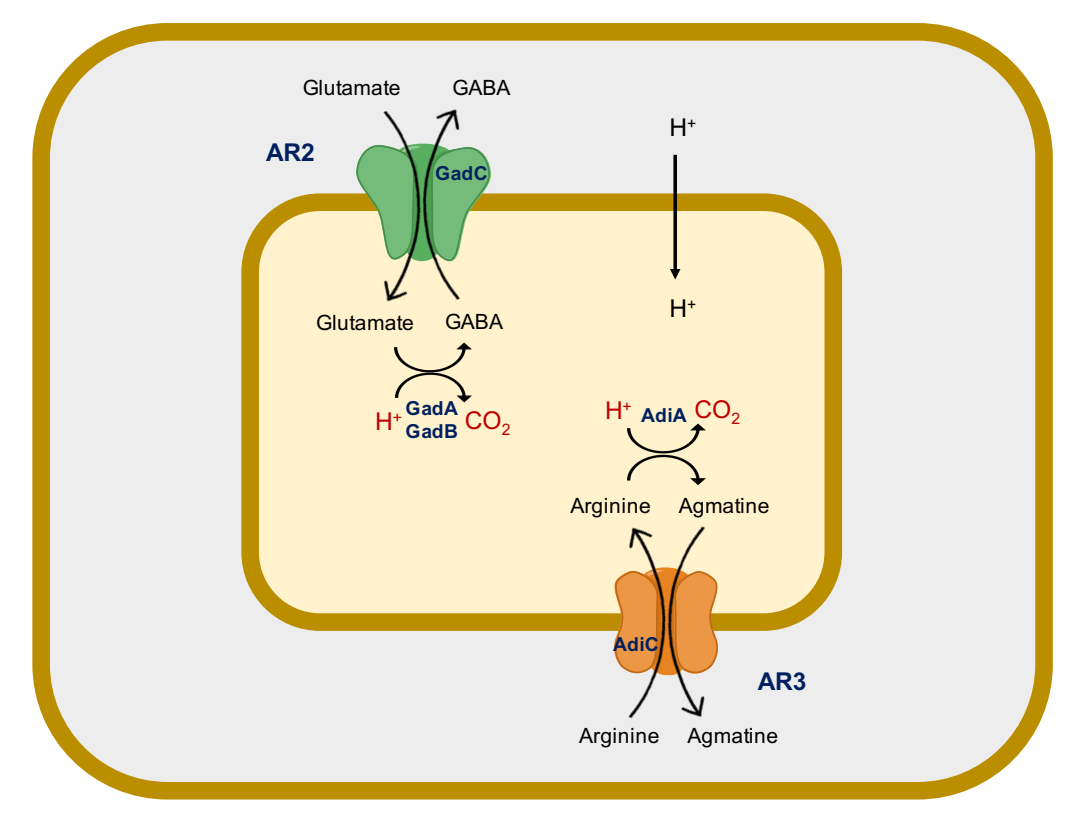

Figure 2.1: Acid resistance systems in $\boldsymbol{E}$. coli. The glutamate- and arginine-dependent acid resistance systems involve the use of hydrogen ions to convert glutamate and arginine to GABA and agmatine respectively as a means of lowering the cytoplasmic $\mathrm{pH}$ within the bacterial cell. Adapted from ( $\mathrm{Lu}$ et al., 2013).

\subsubsection{DNA Damage and DNA Repair Mechanisms}

In addition to its detrimental effects on macromolecules and enzyme function, the low $\mathrm{pH}$ exposure encountered during gastric passage is particularly damaging to bacterial DNA. Specifically, acid stress causes DNA damage primarily through depurination and depyrimidation and to a lesser extent through the formation of double-stranded lesions in the DNA, with the level of DNA damage increasing linearly with a decrease in pH (Calhoun and Kwon, 2011; Choi et al., 2000; Lindahl and Nyberg, 1972). These alterations in DNA lead to unrepaired DNA and mismatches in repaired sequences, which can prove to be fatal to the cell (Choi et al., 2000; Raja et al., 1991; Thompson et al., 1998). Consequently, the ability of bacteria to repair the damaged DNA represents a major survival strategy for bacteria to withstand passage through extreme acidic conditions such as gastric acid (Choi et al., 2000). Studies have demonstrated that mutations in 
DNA repair mechanisms result in significant decreases in acid tolerance of bacteria, thereby confirming that the ability to repair DNA damage is a vital aspect to survival in acidic environments (Choi et al., 2000; Thompson et al., 1998).

When DNA is damaged through the loss of a purine or pyrimidine base, the form of DNA repair utilized by bacterial cells is site-specific recombination (Figure 2.2). During $\lambda$ integrasemediated site-specific recombination, the Int protein alongside additional accessory factors places the two recombination substrates close together in a synaptic complex. The active site of each Int monomer then attacks a specific phosphodiester linkage and forms a transient covalent 3'phosphotyrosyl bond between the enzyme and the top strand of each DNA substrate (Boldt et al., 2004). Ligation occurs when the free 5'-OH group from an adjacent substrate acts as a nucleophile at this phosphotyrosyl linkage. Since the two DNA strands of each substrate are cleaved independently, a Holliday junction (HJ) is generated as a critical intermediate structure during recombination. The complex then undergoes an isomerization, which positions the previously inactive Int monomers into the appropriate confirmation for subsequent catalytic steps. To resolve the HJ, a second set of DNA cleavage, strand exchange, and ligation steps on the bottom strand of each DNA substrate occurs, resulting in two recombinant DNA molecules (Boldt et al., 2004; Kepple et al., 2005; Lino et al., 2011).

The ability of bacterial cells to successfully repair DNA damage, and specifically the ability to resolve the HJ intermediate structure, using this mechanism is a critical component to surviving acute acid exposure, and ensures pathogen survival past the stomach and into the intestine where colonization occurs. In contrast, the DNA repair process also creates a significant vulnerability for the bacterial cells, since failure to effectively repair the DNA results in bacterial 
cell death. While this provides a large obstacle for the pathogen, it also creates a unique opportunity to exploit this vulnerability as a means of increasing bacterial cell death during gastric passage.

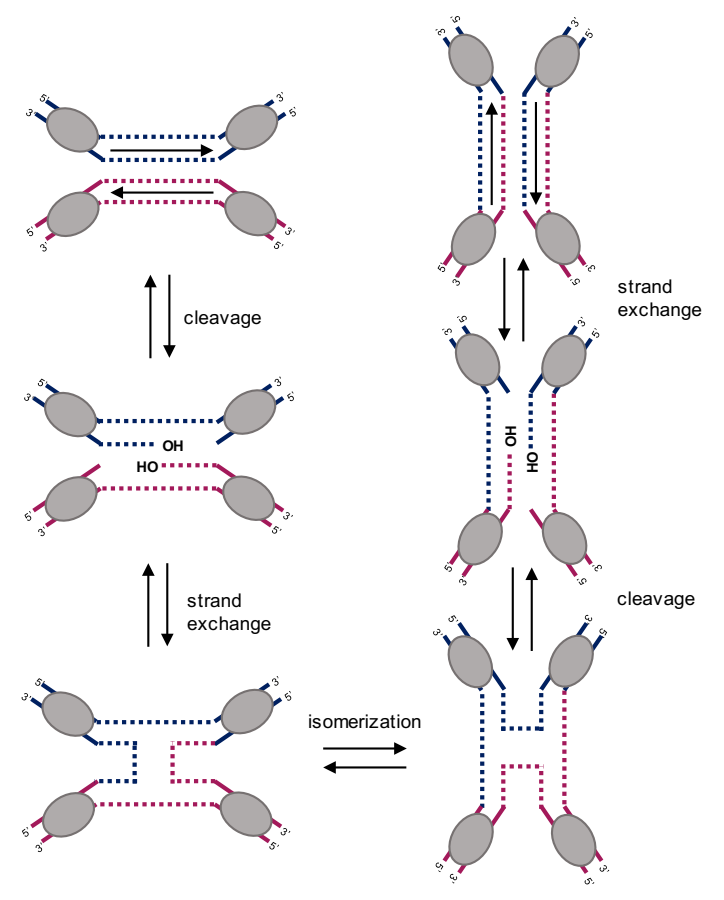

Figure 2.2: Catalytic events mediated by Int during site-specific recombination. A holliday junction is generated after the first round of DNA cleavage, strand exchange, and ligation, and is resolved in a second round of the same catalytic steps. Adapted from (Boldt et al., 2004).

\subsubsection{Antimicrobial Peptide wrwycr}

\subsubsection{Mode of Action}

The antimicrobial peptide wrwycr is a D-amino acid hexapeptide, originally identified in a screen of chemically synthesized inhibitors of $\lambda$ integrase-mediated recombination (Boldt et al., 2004; Lino et al., 2011). The active oxidized form of the peptide is a dimer linked through a disulphide bridge which connects the cysteine present in each peptide monomer (Boldt et al., 2004; Kepple et al., 2005). 
The peptide acts as an inhibitor of branched DNA structures such as HJs and collapsed replication forks, albeit with lesser affinity for collapsed replication forks (Sharples et al., 1999; Whitby et al., 1993). More specifically, peptide wrwycr is a potent inhibitor of RecG and the RuvABC complex - enzymes which play a vital role in HJ and replication fork repair (Flores, 2001; Kepple et al., 2005; Michel et al., 2004). RecG is a monomeric DNA helicase that aids in HJ resolution via branch migration by unwinding the leading and lagging strands simultaneously prior to them reannealing to each other (Whitby et al., 1993; Whitby and Lloyd, 1998). Similarly, the RuvABC complex also acts to resolve HJs in a manner distinct from RecG. In this model, RuvAB binds to the $\mathrm{HJ}$ to open it up into a square planar conformation, thus allowing for the RuvC resolvase to bind and cleave the leading and lagging strands to unwind and reanneal (Kepple et al., 2005).

Interestingly, the preferred substrate for each of RecG, RuvC, and peptide wrwycr is HJs opened into a square planar conformation (Sharples et al., 1999; Sharples, 2001). In fact, the peptide binds with 10-100X higher affinity to HJs in a square planar conformation than those present as stacked $\mathrm{X}$ junctions. This is likely due to the square planar conformation opening up the junction and allowing more space in the center for binding of the peptide (Kepple et al., 2005). Since the peptide binds to the same site on the HJ, it follows that it prevents binding of either RecG or RuvC thereby inhibiting their action to resolve HJ resolution (Figure 2.3) (Kepple et al., 2005). 

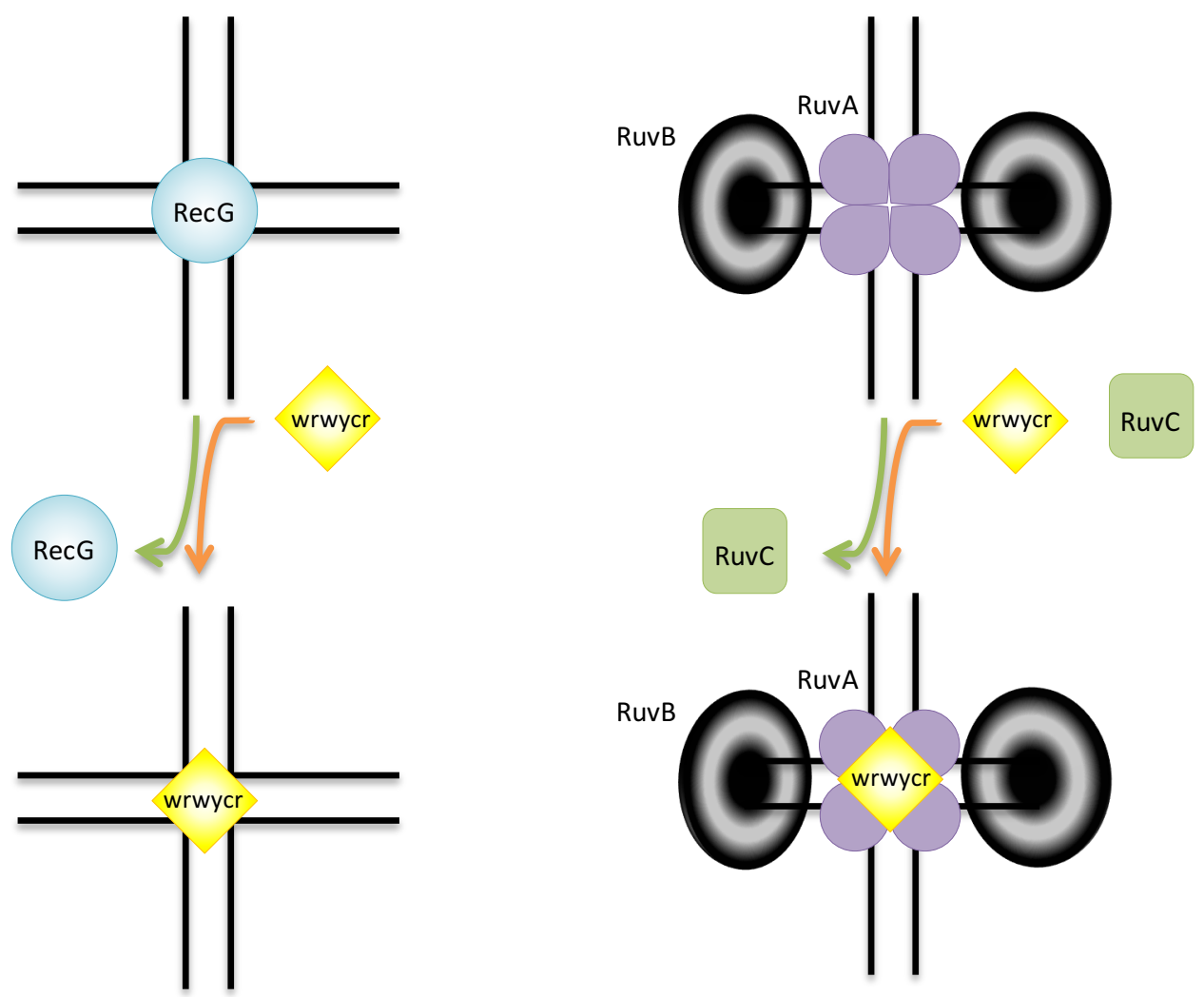

Figure 2.3: Peptide wrwycr mode of action. Antimicrobial peptide wrwycr binds to the square planar conformation of $\mathrm{HJs}$, thereby acting as a competitive inhibitor of RecG and RuvC - enzymes independently involved in HJ resolution.

\subsubsection{Peptide wrwycr as an Antimicrobial}

Together, the necessity of $\mathrm{HJ}$ resolution for cell viability and the mode of action of the peptide wrwycr provides an exciting potential for this peptide as an antimicrobial compound. Results have demonstrated that the peptide has bactericidal activity through its interference of intracellular target activity (Gunderson and Segall, 2006). However, the exact mechanism through which the peptide enters bacterial cells remains under investigation as results have demonstrated support for both passive and active transport (Su et al., 2010). It has been postulated that active transport may be facilitated through endocytosis of the peptide, resulting in a higher concentration of peptide inside the cell. In contrast, the peptide's small size and hydrophobicity may allow it to 
passively diffuse through both lipid bilayer membranes into the cytoplasm (Lino et al., 2011; Su et al., 2010). Similarly, it has been suggested that the peptide may act similarly to other cationic antimicrobial peptides which enter the cell by forming pores in the membrane, causing an increase in membrane permeability (Brogden, 2005; Brown and Hancock, 2006). However wrwycr contains no predicted alpha helix forming domains as is seen in other pore-forming antimicrobials, which conflicts with this theory. A more likely scenario stems from the fact that the peptide's amino acid composition resembles that of the cell-penetrating class of peptides (CPPs). CPPs are characterized by their amphipathic nature and their positive charge at physiological $\mathrm{pH}$, and utilize both endocytic mechanisms and temperature- and endocytosis-independent membrane translocation for entry into cells ( $\mathrm{Su}$ et al., 2010). Additionally, the growth media in which the peptide is introduced to the bacteria in may affect bacterial sensitivity to peptide treatment by limiting uptake of the peptide. This may be due to the peptide's interaction with the bacterial membrane being salt sensitive, thereby requiring low salt media for efficient peptide uptake, as is seen with other classes of antibiotics (Goldman et al., 1997; Tam, 2002).

Once inside the cell, the peptide has been shown to display bactericidal activity against both gram-positive and gram-negative bacteria, primarily through the accumulation of DNA breaks as a result of HJ resolution inhibition (Gunderson and Segall, 2006). This in turn leads to filamentation of cells, chromosome segregation defects, and anucleate cells. Results have also demonstrated that peptide treatment is effective at inhibiting growth of Salmonella present within macrophage-like cells and murine peritoneal macrophages, and does not affect metabolic activity

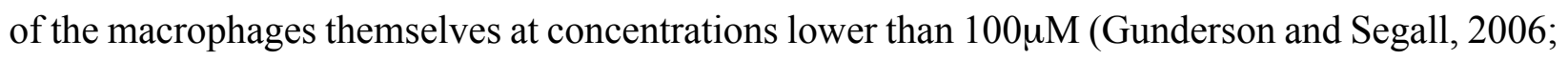
Su et al., 2010). Interestingly, the peptide WRWYCR, composed of L-amino acids, displays a similar potency in inhibiting $\mathrm{HJ}$ resolution and has therefore displayed a similar level of 
bactericidal activity against non-pathogenic E. coli. However, when tested in vivo, wrwycr shows a greater level of antimicrobial activity, likely due to the D-amino acids that renders it resistant to degradation by peptidases (Gunderson and Segall, 2006; Kepple et al., 2005, 2008).

In addition to the effects on $\mathrm{HJ}$ resolution, peptide treatment has also been shown to limit bioavailable iron but increase chelatable iron within the cell in a $\mathrm{pH}$-independent manner, as seen by qPCR analysis of Fur-regulated genes and electron paramagnetic resonance spectroscopy (Orchard et al., 2012). The explanation for the lower levels of bioavailable iron present within peptide-treated cells is unclear, however, the authors suggested that it may be a result of the peptide either directly chelating iron $\left(\mathrm{Fe}^{3+}\right)$ weakly or indirectly sequestering iron through binding of a siderophore. Due to this reduction in bioavailable iron, and evidence that E. coli mutants defective in enterobactin synthesis and ferric enterobactin uptake were hypersensitive to peptide wrwycr treatment, the authors suggested that the ability of cells to take up ferric enterobactin from the external environment is vital for E. coli to withstand peptide treatment (Orchard et al., 2012). In contrast, the increase in chelatable iron $\left(\mathrm{Fe}^{3+}\right)$ may be explained by the peptide acting similarly to other bactericidal antibiotics that break down iron-sulfur clusters within the cell, thereby releasing iron $\left(\mathrm{Fe}^{2+}\right)$ into the cell and potentially leading to iron toxicity (Calhoun and Kwon, 2011; Kohanski et al., 2007).

More recently, the combination of peptide treatment and acute acid stress representative of gastric passage showed significantly enhanced levels of STEC killing in seropathotypes most highly associated with HUS infection (Lino et al., 2011). This result is likely due to an increase in DNA damage caused as a result of acute acid stress which in turn leads to an increase in HJ formation during the DNA repair process. The peptide is then free to bind the open HJs and inhibit resolution through blocking RecG and RuvC binding, thereby leading to an increase in cell death 
(Lino et al., 2011). Interestingly, while peptide treatment has previously been associated with an increase in the SOS response of bacteria ( $\mathrm{Su}$ et al., 2010), the combination of peptide-acid treatment showed no increase in Shiga toxin production, a finding that suggests a reduced risk of progression to HUS. These findings provide an exciting opportunity for the use of peptide wrwycr as a preventative strategy for EHEC infection, by targeting the pathogen during gastric passage before it reaches the site of colonization in the large intestine.

\subsubsection{Citrobacter rodentium as a Model of EHEC Infection}

\subsubsection{Comparison to EHEC}

When studying human GI disease such as inflammatory bowel disease and colitis, mice have become the preferred small animal model as they have similar GI tracts to their human counterparts. However, since EHEC is a human-specific pathogen, it does not readily colonize the mouse GI tract (Lenz et al., 2015; Wiles et al., 2006a). This can be manipulated by infecting mice with extremely high doses of EHEC, or by using germ-free or streptomycin-treated mice, however these models do not give an accurate biological representation of infection (Rodrigues et al., 2012; Smith and Bhagwat, 2013). To better understand the biological aspect of EHEC infection, a well established model has been developed that employs the closely related murine pathogen Citrobacter rodentium (C. rodentium) (Lenz et al., 2015; Rodrigues et al., 2012; Wiles et al., 2006a). The two most commonly used strains are ICC168 and DBS100, both of which were isolated from a diarrhea outbreak in mice at Yale University School of Medicine in 1972 (Lenz et al., 2015).

Similarly to EHEC, C. rodentium colonizes the intestinal mucosa in mice and is characterized by the formation of attaching and effacing lesions that allow for a more intimate 
bacterial attachment to the host (Collins et al., 2014; Frankel et al., 1998; Frankel and Phillips, 2008). At the genetic level, C. rodentium and EHEC share $67 \%$ of their genes including the LEE pathogenicity island which encodes various effector proteins along with the T3SS that functions as a molecular syringe used to directly inject bacterial effector proteins into the host cytoplasm such as Tir, EspF, EspG, EspB, NleB, NleC, and NleH (Arbeloa et al., 2009; Collins et al., 2014; Mundy et al., 2004; Petty et al., 2010). C. rodentium does not produce Shiga toxin, characteristic of STEC infection, although a strain that expresses stx has recently been constructed, thereby providing a convenient animal model for studying how the toxin contributes to pathogenesis in vivo (Collins et al., 2014; Mallick et al., 2012). Nevertheless, C. rodentium has been established as the gold standard small animal model for investigating the virulence mechanisms of $\mathrm{A} / \mathrm{E}$ pathogens such as EHEC as well as human intestinal diseases such as Crohn's disease, ulcerative colitis, and colon tumorigenesis (Chandrakesan et al., 2014; Collins et al., 2014; Higgins et al., 1999).

\subsubsection{Murine Infection Cycle and Infection Outcomes}

C. rodentium infection studies involve orogastric gavage of mice with laboratory-cultured bacteria, resulting in a highly reproducible infection model (Collins et al., 2014). Once inoculated, C. rodentium initially colonizes the caecal patch in the caecum although the vast majority of bacteria pass straight through the GI tract. The adherent bacteria adapt to the environmental conditions present within the GI tract which facilitates a virulence switch to promote colonization of the distal colon and rectum (Collins et al., 2014; Wiles et al., 2004, 2006b). Once infected, mice develop colitis (also known as transmissible murine crypt hyperplasia) although the severity of colitis is dictated by the genetic background of the host mice. In some cases, infection is fatal 
resulting in death, whereas in other cases it causes self-limited inflammation of the caecum and colon (Borenshtein et al., 2008, 2009; Higgins et al., 1999; Mundy et al., 2005; Papapietro et al., 2013; Wiles et al., 2006a). Additionally, infection causes dysbiosis in the GI microbiota which is characterized by an overgrowth of $C$. rodentium to approximately $1-3 \%$ of the total microbiota and a subsequent reduction in the overall abundance and diversity of the resident microbiota (Clare et al., 2013; Hoffmann et al., 2009; Lupp et al., 2007). Other infection outcomes include soft stool and loss of barrier integrity. Following the peak in bacterial infection, clearance of the pathogen begins as colonized epithelial cells are shed into the intestinal lumen until complete clearance occurs 2-3 weeks post-infection (Collins et al., 2014; Wiles et al., 2004, 2006b).

\subsubsection{Rationale and Overall Aim}

Since EHEC infections are a continuous global problem, with new outbreaks occurring almost every year, and traditional treatments have been ineffective it is important to develop new therapeutic strategies, particularly preventative strategies that target E. coli before it is able to colonize and infect the host. The combination of peptide pre-treatment followed by acute acid stress represents a unique opportunity to enhance one of the human body's natural defenses against incoming pathogens by exploiting the vulnerability it causes in EHEC. Since previous studies have already demonstrated the efficacy of peptide-acid treatment on pathogen killing in vitro, the next logical step would be to test its efficacy in vivo using a suitable animal model of infection. Consequently, the use of the murine-specific pathogen $C$. rodentium provides a well established and reproducible mouse model for EHEC infection. While a disadvantage to the use of $C$. rodentium lies in its inability to produce the characteristic Shiga toxin associated with Shiga toxinproducing strains, the fact that peptide-acid treatment showed no effect on Stx production (Lino et 
al., 2011) suggests that the use of the $C$. rodentium mouse model of infection is an appropriate model for studying the efficacy of peptide treatment. In summary, the goal of this research project is to test the potential of the antimicrobial peptide wrwycr as a prevention strategy against EHEC infection using the $C$. rodentium mouse model of infection.

\subsection{MATERIALS AND METHODS}

\subsubsection{Bacterial strains and culture conditions}

Citrobacter rodentium strain DBS 100 (kindly provided by the late David Schauer, Massachusetts Institute of Technology, Cambridge, MA) was used for in vitro and in vivo infection studies (Rodrigues et al. 2012). Bacterial glycerol stocks were maintained at $-80^{\circ} \mathrm{C}$, and were streaked onto Luria-Bertani (LB) (BioShop, Burlington, ON, Canada) agar prior to use in order to obtain single colonies. Overnight cultures were prepared by inoculating single colonies into LB broth and then incubating $12-16 \mathrm{hr}$ at $37^{\circ} \mathrm{C}$ with shaking.

\subsubsection{Antimicrobial peptide wrwycr}

The peptide wrwycr was synthesized with a C-terminal amide group, purified to $>95 \%$ purity at Sigma-Genosys or Biosynthesis and dissolved in 50\% dimethyl sulphoxide (DMSO), as described previously (Gunderson and Segall, 2006). A wrwycr stock solution (10mM) was maintained at $-20^{\circ} \mathrm{C}$ in 50 or $100 \%$ DMSO. 


\subsubsection{In vitro survival assays}

To assess the impact of acute acid stress on C. rodentium survival, overnight bacterial cultures of $C$. rodentium were diluted 1:10 in fresh LB broth and grown $1-2 \mathrm{hr}$ at $37^{\circ} \mathrm{C}$ with shaking to mid-exponential phase $\left(\mathrm{OD}_{600} 0.4-0.6\right)$. Bacteria were pelleted at 3,500 rpm for $10 \mathrm{~min}$ at $4{ }^{\circ} \mathrm{C}$, re-suspended in an equal volume of $\mathrm{LB}$ broth at the appropriate $\mathrm{pH}(\mathrm{pH} 3.0,3.5,4.0,5.0,6.0$, or 7.0; adjusted with $\mathrm{HCl}$ ), and then incubated statically at $37^{\circ} \mathrm{C}$ for $3 \mathrm{hrs} .10 \mu \mathrm{L}$ samples were taken at $\mathrm{t}=0,1,2$, and $3 \mathrm{hrs}$, serially diluted in $1 \mathrm{X}$ phosphate-buffered saline (PBS) $(137 \mathrm{mM} \mathrm{NaCl}$, 2.7mM KCl, $10 \mathrm{mM} \mathrm{Na}_{2} \mathrm{HPO}_{3}, 1.8 \mathrm{mM} \mathrm{KH}_{2} \mathrm{PO}_{4}$ ), plated onto $\mathrm{LB}$ agar and grown overnight at $37^{\circ} \mathrm{C}$.

For the determination of $C$. rodentium survival after peptide treatment, overnight bacterial cultures of $C$. rodentium were diluted 1:10 in fresh LB broth and grown $1-2 \mathrm{hr}$ at $37^{\circ} \mathrm{C}$ with shaking to mid-exponential phase. Bacteria were pelleted as above and re-suspended in an equal volume of $0.5 \mathrm{X}$ PBS $\left(68.5 \mathrm{mM} \mathrm{NaCl}, 1.35 \mathrm{mM} \mathrm{KCl}, 5 \mathrm{mM} \mathrm{Na}_{2} \mathrm{HPO}_{3}, 0.9 \mathrm{mM} \mathrm{KH}_{2} \mathrm{PO}_{4}\right)$, containing either $0,25,50$, or $65 \mu \mathrm{M}$ of peptide wrwycr or placebo peptide wkhyny. Re-suspended bacteria were incubated for $1 \mathrm{hr}$ at room temperature, static. $10 \mu \mathrm{L}$ samples were taken at t $=0,15,30,45$, and 60 mins, serially diluted in $1 \mathrm{X}$ PBS, plated onto LB agar and grown overnight at $37^{\circ} \mathrm{C}$.

To evaluate $C$. rodentium survival after peptide pre-treatment followed by acute acid treatment, overnight cultures were diluted 1:10 in fresh LB broth and grown $1-2 \mathrm{hr}$ at $37^{\circ} \mathrm{C}$ with shaking to mid-exponential phase. Bacteria were pelleted as above and re-suspended in an equal volume of $0.5 \mathrm{X}$ PBS containing either $0,25,50$, or $65 \mu \mathrm{M}$ of peptide wrwycr. Re-suspended bacteria were incubated for $15 \mathrm{~min}$ at room temperature and then pelleted as above. Bacterial pellets were then re-suspended in PBS at pH $3.5\left(500 \mathrm{mM} \mathrm{KH}_{2} \mathrm{PO}_{4}, 164 \mathrm{mM} \mathrm{NaCl}\right.$; adjusted with phosphoric acid) and incubated at $37^{\circ} \mathrm{C}$ for $1 \mathrm{hr}$. Samples were taken at $\mathrm{t}=\mathrm{UT}$ (untreated; 
immediately prior to addition of peptide), 0 (immediately prior to addition of acid), 15, 30, 45, and 60 mins, serially diluted in $1 \mathrm{X}$ PBS, plated onto LB agar and grown overnight at $37^{\circ} \mathrm{C}$.

\subsubsection{Growth phase dependency assay}

Overnight cultures of C. rodentium were diluted 1:10 in fresh LB broth and grown 0-4 hr at $37^{\circ} \mathrm{C}$ with shaking to either lag phase $\left(\mathrm{OD}_{600} 0.1\right)$, mid-exponential phase $\left(\mathrm{OD}_{600} 0.4-0.6\right)$, or stationary phase $\left(\mathrm{OD}_{600} 0.8-1.0\right)$. Bacteria were pelleted as above and re-suspended in an equal volume of $0.5 \mathrm{X}$ PBS containing 50 $\mu \mathrm{M}$ of peptide wrwycr. Re-suspended bacteria were incubated at room temperature for $1 \mathrm{hr}$, static. Samples were taken at $\mathrm{t}=1 \mathrm{hr}$, serially diluted in $1 \mathrm{X}$ PBS, plated onto LB agar and grown overnight at $37^{\circ} \mathrm{C}$.

\subsubsection{Biofilm formation and survival assays}

Biofilms were formed by inoculating $1 \mathrm{~mL}$ of a 1:1000 dilution of overnight culture in fresh LB broth into 12-well plates (Greiner Bio-One, Monroe, NC, USA). Plates were incubated at $37^{\circ} \mathrm{C}$ under static growth conditions for $48 \mathrm{hrs}$, with $1 \mathrm{~mL}$ fresh LB broth being replaced every 12-16 hrs. Crystal violet staining and confocal microscopy were carried out on $48 \mathrm{hr}$ biofilms to ensure sufficient biofilm growth. Once the biofilms were formed, it was washed twice with 1X PBS to remove non-adherent cells. $0.5 \mathrm{X}$ PBS containing the appropriate concentration of peptide $(0,25$, 50 , or $65 \mu \mathrm{M}$ ) was then added to each well and incubated at room temperature for 30 mins. After incubation, wells were washed once with 1X PBS and bacteria were scraped off the bottom of each well and suspended in 1X PBS. Bacterial cell viability was assessed by preparing serial dilutions in 1X PBS and plating onto LB agar overnight at $37^{\circ} \mathrm{C}$. 


\subsubsection{Growth recovery after peptide and peptide-acid treatment}

To assess the ability of $C$. rodentium to recover from peptide treatment, overnight cultures were diluted to an $\mathrm{OD}_{600}$ of 0.05 in Mueller Hinton Broth (MHB) (SigmaAldrich, Oakville, ON, Canada) containing either $0,25,50$, or $65 \mu \mathrm{M}$ of peptide wrwycr. MHB broth was used as a minimal media to allow efficient uptake of the peptide by the bacteria while still providing a sufficient source of nutrients. Re-suspended bacteria were incubated statically for $50 \mathrm{hr}$ at $37^{\circ} \mathrm{C}$. Samples were taken every 5 hrs, serially diluted in 1 X PBS, and plated onto LB agar overnight at $37^{\circ} \mathrm{C}$.

In order to assess the ability of $C$. rodentium to recover from peptide-acid treatment, bacteria were diluted 1:10 in fresh LB broth and grown at $37^{\circ} \mathrm{C}$ with shaking to mid-exponential phase $\left(\mathrm{OD}_{600}\right.$ 04-0.6). Bacteria were then pelleted, as described above, and then pre-treated with either $0,25,50$, or $65 \mu \mathrm{M}$ of peptide wrwycr followed by $1 \mathrm{hr}$ acid stress (pH 3.5), as described earlier. Following peptide-acid treatment, bacteria were pelleted as above, re-suspended in an equal volume of MHB broth and incubated statically for 5 hours at $37^{\circ} \mathrm{C}$. Samples were taken every hour, serially diluted in $1 \mathrm{X}$ PBS and plated onto LB agar overnight at $37^{\circ} \mathrm{C}$.

\subsubsection{Resistance assays}

To assess the ability of peptide pre-treatment of $C$. rodentium to induce resistance to further peptide treatment, overnight cultures were diluted to an $\mathrm{OD}_{600}$ of 0.05 in $\mathrm{MHB}$ broth containing either $0,25,50$, or $65 \mu \mathrm{M}$ of peptide wrwycr. Re-suspended bacteria were incubated statically for $24 \mathrm{hr}$ at $37^{\circ} \mathrm{C}$. Treated bacteria were then pelleted as above, and re-suspended in an equal volume of $0.5 \mathrm{X}$ PBS containing 50 $\mathrm{MM}$ peptide. Re-suspended bacteria were incubated statically for $1 \mathrm{hr}$ at room temperature. Samples were taken after the initial $24 \mathrm{hr}$ of pre-treatment, and following the 
$1 \mathrm{hr}$ of treatment in fresh peptide, serially diluted in 1X PBS and plated onto LB agar overnight at $37^{\circ} \mathrm{C}$.

\subsubsection{Mouse infection model}

Six week old female C57BL/6 mice (Jackson Laboratory) were used for in vivo studies. Mice were given free access to sterile drinking water and chow for the duration of the study. To assess the safety of peptide administration to mice, animals were administered either peptide wrwycr or placebo wkhyny alone at the 3 concentrations being tested $(25,50$, or $65 \mu \mathrm{M})$ via orogastric gavage. Mice were sacrificed on day 10 post-administration, with the distal colon excised and placed into neutral-buffered formalin for histological analysis.

For the evaluation of peptide safety in vivo, mice were divided into six experimental groups: (1) sham-infected, (2) C. rodentium infected, (3) peptide wrwycr pre-treated C. rodentium infected, (4) peptide alone, (5) placebo wkhyny pre-treated C. rodentium infected, (6) placebo alone. Infection with $10^{8} \mathrm{CFU} C$. rodentium was delivered by orogastric gavage $(100 \mu \mathrm{L})$, as previously described (Gareau et al, 2011; Rodrigues et al, 2012). Peptide and placebo (25, 50, and $65 \mu \mathrm{M})$ pre-treated $C$. rodentium groups were pre-treated for 5 minutes at room temperature prior to orogastric gavage.

Mice were observed daily for overall health and well-being throughout the duration of the study protocol. The presence of fur ruffling, diarrhea, lethargy, and changes in weight were used as indicators of ill health (Gareau et al, 2011; Rodrigues et al 2012). Mice were sacrificed at the height of infection, on day 10 post-infection, with the distal colon excised and placed into neutralbuffered formalin. Fecal pellets were collected on days 0, 6, and 10 post-infection and stored at 
$-80^{\circ} \mathrm{C}$. Fecal swabs were collected and plated onto MacConkey agar on days 0, 6, and 10 postinfection and incubated overnight at $37^{\circ} \mathrm{C}$ prior to scoring the level of growth. All procedures and protocols were approved by the Animal Care Committee at the Hospital for Sick Children, Toronto, ON.

\subsubsection{Histological analysis of colon samples}

Colonic epithelial hyperplasia in response to peptide safety and C. rodentium infection with or without peptide pre-treatment was observed on coded segments of distal colon, as described

previously (Rodrigues et al, 2012). Briefly, distal colon sections were excised at the time of sacrifice, fixed in 10\% neutral-buffered formalin, and embedded in paraffin. Sections were stained with hematoxylin and eosin and visualized using a Leica DM5000B microscope, Leica DFC350 FX camera, and ImageJ software. Crypt cell lengths were measured on coded segments, with findings presented as the mean of 10 crypts per section from 3 non-adjacent sections per animal.

\subsubsection{0 qPCR analysis of $C$. rodentium infection}

Fecal pellets were collected at day 10 post-infection and quantitative PCR (qPCR) was used to probe for the presence of the espB gene of $C$. rodentium as previously described (Livak and Schmittgen, 2001; McKeel et al, 2002). Briefly, fecal pellets were collected and stored at $80^{\circ} \mathrm{C}$ until use. Bacterial DNA was extracted using the QIAamp DNA Stool Kit (Qiagen, Toronto, ON, Canada). A Nanodrop 2000c spectrophotometer was used to measure the concentration and purity (A260/A280) of isolated DNA. DNA was diluted to $5 \mathrm{ng} / \mathrm{mL}$ and frozen at $-20^{\circ} \mathrm{C}$. $\mathrm{qPCR}$ was performed on a CFX96 C1000 Thermal Cycler (BioRad, Mississauga, ON, Canada) using SsoFast EvaGreen supermix according to the manufacturer's instructions. C. rodentium was 
quantified relative to total bacteria using previously published primers specific to $\operatorname{esp} B$ (Table 2.1) (Livak \& Schmittgen, 2001; McKeel et al, 2002).

\subsubsection{1 qPCR analysis of alterations in stool microbial communities}

The fecal microbiota was analyzed using $16 \mathrm{~S}$ rRNA qPCR analysis. Briefly, fecal pellets were collected and stored at $-80^{\circ} \mathrm{C}$ until use. Bacterial DNA was extracted using the QIAamp DNA Stool Kit. A Nanodrop 2000c spectrophotometer was used to measure the concentration and purity (A260/A280) of isolated DNA. DNA was diluted to $5 \mathrm{ng} / \mathrm{mL}$ and frozen at $-20^{\circ} \mathrm{C}$. qPCR was performed on a CFX96 C1000 Thermal Cycler using SsoFast EvaGreen supermix according to the manufacturer's instructions. Levels of Bacteriodetes, Firmicutes, Gammaproteobacteria, and Lactobacillus were quantified relative to total bacteria using previously published primers (Table

2.1) (Bacchetti de Gregoris et al., 2011). Data analyses were performed, as previously described (Livak and Schmittgen, 2001; Pfaffl, 2001).

Table 2.1: Primer sequences used for quantitative PCR of microbial communities.

\begin{tabular}{|c|c|c|c|}
\hline Target Group & $\begin{array}{l}\text { Primer } \\
\text { Name }\end{array}$ & Sequence $\left(5^{\prime} \rightarrow 3^{\prime}\right)$ & Reference \\
\hline \multirow{2}{*}{$\begin{array}{l}\text { Citrobacter } \\
\text { rodentium }\end{array}$} & EspB_F & GCTTCTGCGAAGTCTGTCAA & \multirow{2}{*}{ McKeel, et al., 2002} \\
\hline & EspB_R & CAGTAAAGCGACTTAACAGATT & \\
\hline \multirow{2}{*}{ Universal } & $926 \mathrm{~F}$ & AAACTCAAAKGAATTGACGG & \multirow{2}{*}{$\begin{array}{l}\text { Bacchetti De Gregoris } \\
\text { et al., } 2011\end{array}$} \\
\hline & $1062 \mathrm{R}$ & CTCACRRCACGAGCTGAC & \\
\hline \multirow{2}{*}{$\gamma$-Proteobacteria } & $1080 \gamma \mathrm{F}$ & TCGTCAGCTCGTGTYGTGA & \multirow{2}{*}{$\begin{array}{l}\text { Bacchetti De Gregoris } \\
\text { et al., } 2011\end{array}$} \\
\hline & $\gamma 1202 \mathrm{R}$ & CGTAAGGGCCATGATG & \\
\hline \multirow{2}{*}{ Bacteroidetes } & $798 \mathrm{cfbF}$ & CRAACAGGATTAGATACCCT & \multirow{2}{*}{$\begin{array}{l}\text { Bacchetti De Gregoris } \\
\text { et al., } 2011\end{array}$} \\
\hline & cfb967R & GGTAAGGTTCCTCGCGTAT & \\
\hline \multirow{2}{*}{ Firmicutes } & 928F-Firm & TGAAACTYAAAGGAATTGACG & \multirow{2}{*}{$\begin{array}{l}\text { Bacchetti De Gregoris } \\
\text { et al., } 2011\end{array}$} \\
\hline & 1040FirmR & ACCATGCACCACCTGTC & \\
\hline \multirow{2}{*}{ Actinobacteria } & Act920F3 & TACGGCCGCAAGGCTA & \multirow{2}{*}{$\begin{array}{l}\text { Bacchetti De Gregoris } \\
\text { et al., } 2011\end{array}$} \\
\hline & Act1200R & TCRTCCCCACCTTCCTCCG & \\
\hline
\end{tabular}




\subsubsection{Statistical analyses}

Results are expressed as the mean \pm standard error of the means (SEMs) of 3 independent experiments. Statistical analyses were performed using Student's t test, Mann-Whitney test, oneway or two-way analysis of variance (ANOVA), with Tukey's multiple comparisons testing to determine significance between experimental conditions. A $P$ value $<0.05$ was considered significant.

\subsection{RESULTS}

\subsubsection{Acute acid stress or peptide treatment alone causes moderate killing of $C$. rodentium}

Survival assays of $C$. rodentium over a $\mathrm{pH} 7.0$ to 3.0 range identified $\mathrm{pH} 3.5$ as the optimal $\mathrm{pH}$ for viability loss studies (Figure 2.4a), consistent with the reported $\mathrm{pH}$ of murine gastric acid (McConnell et al., 2008). Survival of $C$. rodentium at acidic versus neutral $\mathrm{pH}$ values showed a significant 3-4 $\log$ fold decrease in $C$. rodentium viability at $\mathrm{pH} 3.5$ after $2-3 \mathrm{hr}$ treatment times $(P$ $<0.05$ ) (Figure 2.4b). These results indicate that $C$. rodentium is sensitive to acid in a time- and pH-dependent manner. 

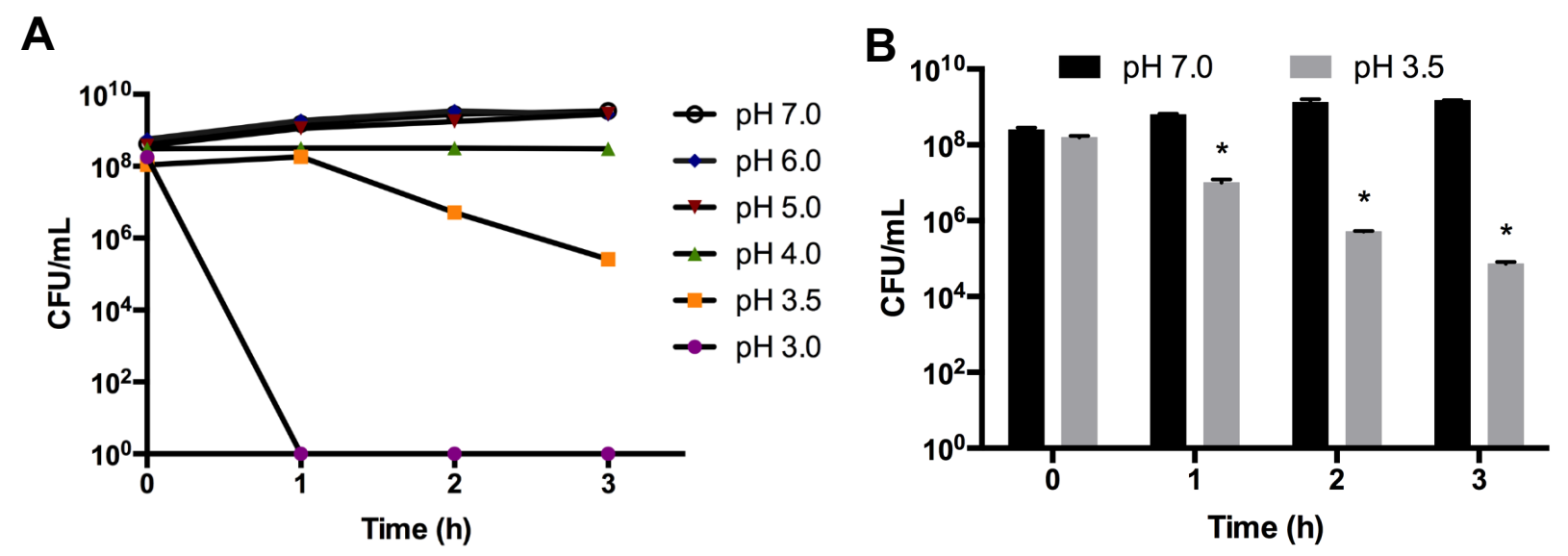

Figure 2.4: Survival of $\boldsymbol{C}$. rodentium following acute acid stress. (a) Survival assay showing CFU/mL over $3 \mathrm{hr}$ acid stress in LB broth at varying pHs at $37^{\circ} \mathrm{C}$. (b) Survival assay showing $\mathrm{CFU} / \mathrm{mL}$ over $3 \mathrm{hr}$ acid stress in $\mathrm{LB}$ broth at $\mathrm{pH} 7.0$ and 3.5 at $37^{\circ} \mathrm{C} . *$ indicates a significant difference compared to $\mathrm{pH} 7.0$ control, $P<0.05$ by two-way ANOVA. Data bars represent means \pm SEMs, $n=3$. Results are from 3 independent trials.

When $C$. rodentium was treated with peptide wrwycr alone, there was a significant timeand dose-dependent decrease in bacterial viability over the course of a 60 min treatment $(P<0.05)$. Viability losses ranged from 2-5 log fold for peptide concentrations of $25-65 \mu \mathrm{M}$ after $60 \mathrm{~min}$ peptide treatment relative to an untreated control (Figure 2.5a). This loss of viability was specific to the antimicrobial peptide wrwycr, and was not seen in a matching placebo peptide wkhyny (Figure 2.5b). These results show that $C$. rodentium is sensitive to peptide treatment, with losses in viability similar to findings previously reported for the human enteric pathogen EHEC O157:H7 (Lino et al, 2011). 

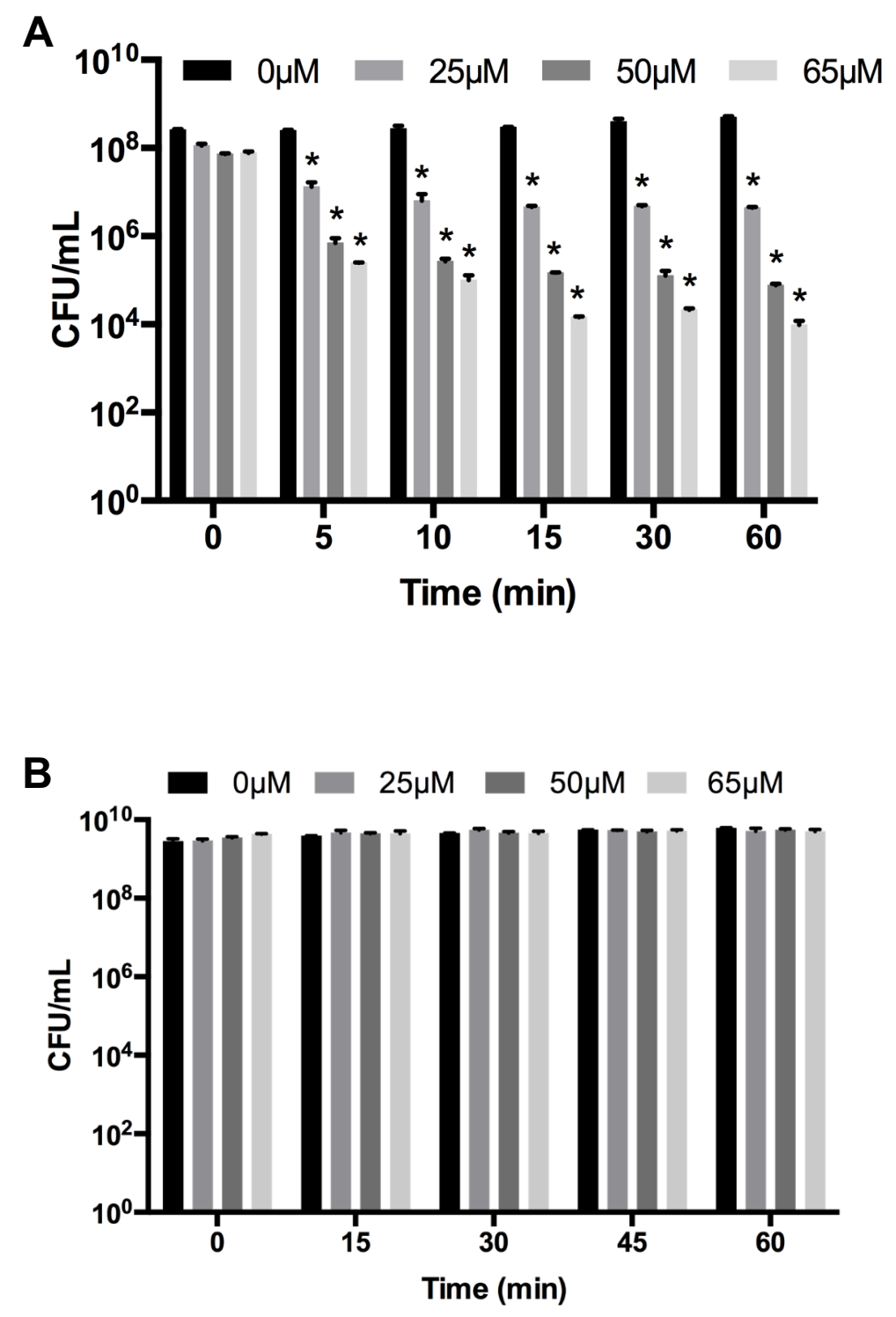

Figure 2.5: Survival of $\boldsymbol{C}$. rodentium following peptide treatment. Survival assay showing $\mathrm{CFU} / \mathrm{mL}$ after 1 hour (a) peptide wrwycr; or (b) placebo wkhyny treatment $(0-65 \mu \mathrm{M})$ at room temperature, static. * indicates a significant difference compared to $0 \mu \mathrm{M}$ control, $P<0.05$ by Student $\mathrm{t}$ test. Data bars represent means \pm SEMs, $n=3$. Results are from 3 independent trials.

Since the majority of experiments were carried out using $C$. rodentium grown to midexponential phase, the effect of growth phase on peptide sensitivity was assessed. The results demonstrated that peptide sensitivity was not significantly different for $C$. rodentium grown to either lag phase, mid-exponential phase, or stationary phase treated with $50 \mu \mathrm{M}$ of peptide for $1 \mathrm{hr}$ 
at room temperature, with approximately $0.01 \%$ of the starting inoculum surviving in all conditions

(Figure 2.6). These results demonstrate that peptide-induced killing of $C$. rodentium is not growthphase specific.

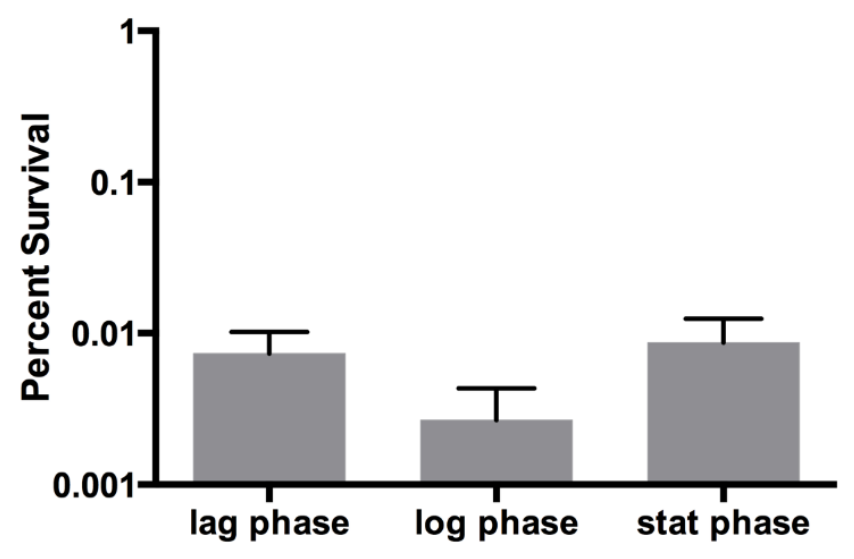

Figure 2.6: Sensitivity of differential growth phases to peptide treatment of $\boldsymbol{C}$. rodentium. Percent survival of $C$. rodentium at either lag, log, or stationary phase after 1 hour peptide treatment $(50 \mu \mathrm{M})$ at room temperature. Data bars represent means \pm SEMs, $n=3$. Results are from 3 independent trials.

\subsubsection{Peptide pre-treatment enhances acid-induced killing of $C$. rodentium}

When C. rodentium was pre-treated with peptide for 5-15 min and then exposed to acute acid stress ( $\mathrm{pH} 3.5)$, there was a time- and dose-dependent decrease in viability over the course of the 60 min acid treatment (Figure 2.7). Reductions in viability ranged from 2-4 $\log$ fold for $C$. rodentium pre-treated with $25-65 \mu \mathrm{M}$ peptide compared to the untreated control, with no detectable C. rodentium observed after $65 \mu \mathrm{M}$ peptide pre-treatment followed by $60 \mathrm{~min}$ of acid stress. These results demonstrate that peptide pre-treatment significantly enhances acid-induced killing $(P<$ 0.05 ), and that survival is significantly lower than survival after either acid or peptide treatment alone. 


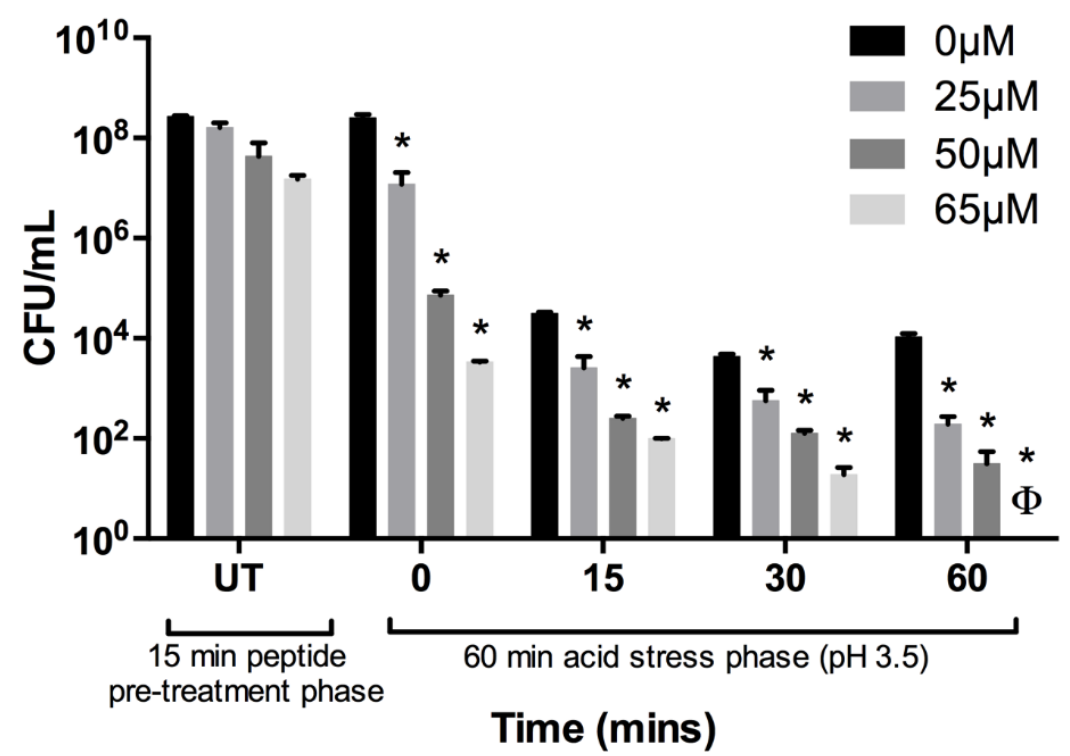

Figure 2.7: Survival of $C$. rodentium following peptide-acid treatment in vitro. Surviving $\mathrm{CFU} / \mathrm{mL}$ of C. rodentium pre-treated with $0-65 \mu \mathrm{M}$ peptide wrwycr for $15 \mathrm{~min}$ at room temperature followed by 1 hour acid stress ( $\mathrm{pH} \mathrm{3.5)}$ at $37^{\circ} \mathrm{C} . *$ indicates a significant difference compared to $0 \mu \mathrm{M}, P<0.05$ by Student $\mathrm{t}$ test. Data bars represent means \pm SEMs, $n=3$. Results are from 3 independent trials.

\subsubsection{Peptide treatment does not impact survival of $C$. rodentium biofilms}

While the focus of this research is planktonic bacteria, we also determined the effect of peptide treatment on established $C$. rodentium biofilms. Confocal microscopy of DAPI-stained biofilms showed proliferation and the formation of microcolonies throughout the biofilm (Figure 2.8a). When the established biofilms were exposed to $25-65 \mu \mathrm{M}$ peptide treatment for $30 \mathrm{~min}$ at room temperature, there was no decrease in viability observed compared to planktonic $C$. rodentium that showed a 2-4 log fold decrease in survival (Figure $\mathbf{2 . 8 b}$ ). These results suggest that established biofilms may be employing resistance mechanisms to prevent bacterial killing through peptide treatment. 

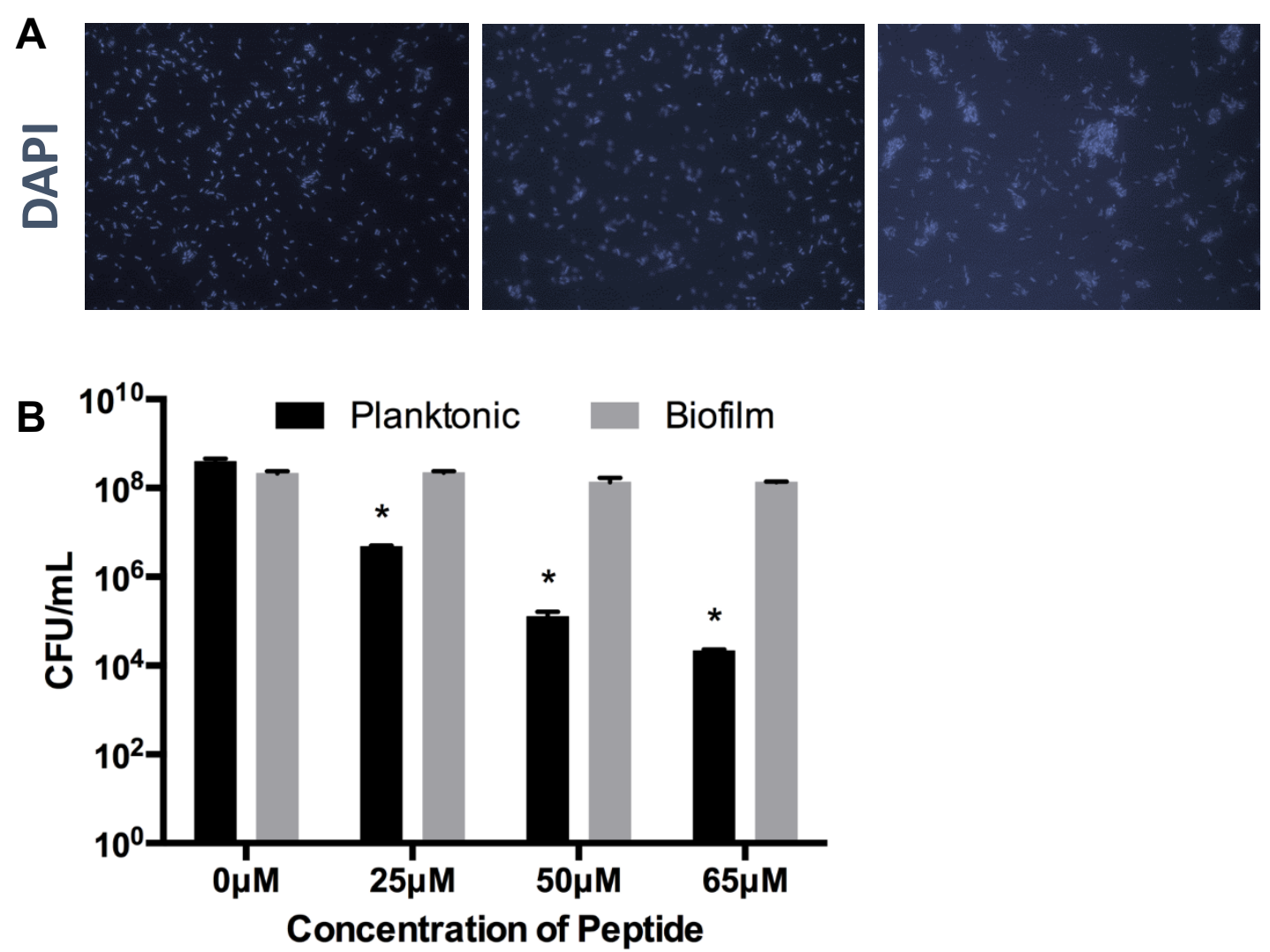

Figure 2.8: Survival of established $C$. rodentium biofilms following peptide treatment. (a) Representative micrographs of DAPI-stained 48 h C. rodentium biofilms. (b) Survival assay showing $\mathrm{CFU} / \mathrm{mL}$ after $0-65 \mu \mathrm{M}$ peptide treatment of established C. rodentium biofilms compared to planktonic cells after 30 min treatment at room temperature. * indicates a significant difference compared to $0 \mu \mathrm{M}, P<0.05$ by two-way ANOVA. Data bars represent means \pm SEMs, $n=3$. Results are from 3 independent trials.

\subsubsection{Growth recovery of peptide- and peptide-acid- treated $C$. rodentium}

We next evaluated growth recovery of $C$. rodentium treated with either peptide alone or pre-treated with peptide followed by acute acid treatment. For C. rodentium treated with peptide alone, there was an expected decrease in survival during the initial $10 \mathrm{hr}$ of treatment in a timeand dose-dependent manner, with evidence of partial to full recovery over the course of $50 \mathrm{hr}$ (Figure 2.9a). C. rodentium growth was either fully recovered ( $25 \mu \mathrm{M}$ peptide) to the level of the control group or partially recovered (50 and $65 \mu \mathrm{M}$ peptide). 
When $C$. rodentium was pre-treated with $25-65 \mu \mathrm{M}$ peptide followed by $1 \mathrm{hr}$ acid stress ( $\mathrm{pH} 3.5)$, the ability to recover was dramatically reduced with no growth recovery detected for 50 and $65 \mu \mathrm{M}$ peptide pre-treated samples and only moderate growth recovery for the $25 \mu \mathrm{M}$ peptide pre-treated samples (Figure 2.9b).

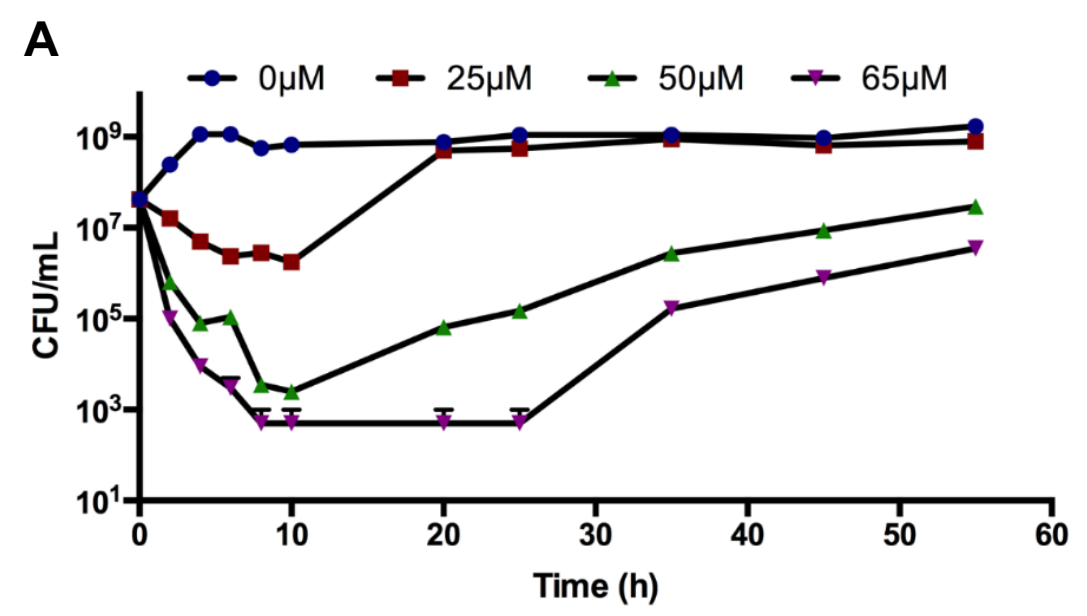

B

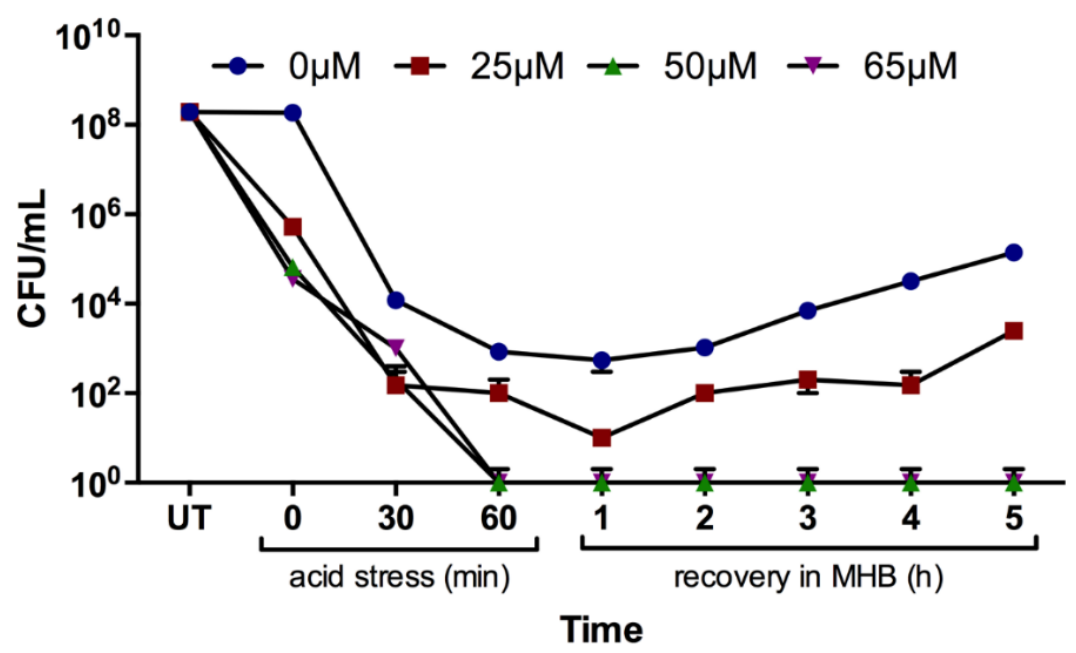

Figure 2.9: Growth recovery of $\boldsymbol{C}$. rodentium in vitro. (a) Recovery of $C$. rodentium growth during prolonged peptide $(0-65 \mu \mathrm{M})$ treatment in $\mathrm{MHB}$ broth at $37^{\circ} \mathrm{C}$, static. (b) Recovery of $C$. rodentium growth after 15 min peptide $(0-65 \mu \mathrm{M})$ pre-treatment followed by $1 \mathrm{hr}$ acid stress $\left(\mathrm{pH} \mathrm{3.5)}\right.$ at $37^{\circ} \mathrm{C}$ in $\mathrm{MHB}$ broth at $37^{\circ} \mathrm{C}$. UT refers to sample taken immediately prior to peptide pre-treatment; 0 min refers to sample taken immediately after peptide pre-treatment, prior to addition of acid stress. Data points represent means \pm SEMs, $n=3$. Results are representative of 3 independent trials. 


\subsubsection{Prior exposure of $C$. rodentium to peptide treatment does not induce resistance}

Since peptide-treated $C$. rodentium was able to achieve either moderate or full growth recovery over prolonged exposure, we tested whether or not this was a result of a resistance phenotype induced by peptide exposure. When $C$. rodentium was pre-treated with either 0 or $25 \mu \mathrm{M}$ peptide wrwycr for $24 \mathrm{hr}$ and then treated with $50 \mu \mathrm{M}$ peptide for $1 \mathrm{hr}$, there was a 2-3 log fold decrease in survival. Additionally, $C$. rodentium that was pre-treated with higher concentrations of peptide, 50 or $65 \mu \mathrm{M}$, showed a $4 \log$ fold decrease in survival when exposed to fresh peptide (Figure 2.10). This increased level of killing at the higher concentrations can be attributed to the lower starting $\mathrm{CFU} / \mathrm{mL}$ of these samples at the start of the $50 \mu \mathrm{M}$ treatment. Since the samples that were pre-treated with peptide prior to treatment with $50 \mu \mathrm{M}$ fresh peptide did not show a significant increase in survival compared to the sample that was not pre-treated with peptide, it can be concluded that prior exposure to peptide up to $24 \mathrm{~h}$ does not induce a resistance phenotype in $C$. rodentium.

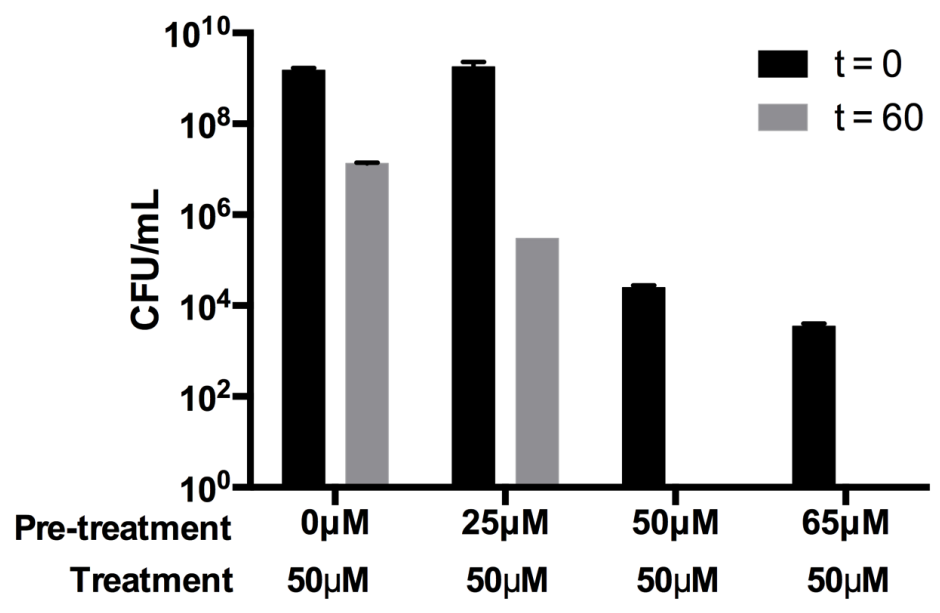

Figure 2.10: Resistance of pre-exposed $C$. rodentium to fresh peptide. Survival of pre-treated $C$. rodentium after $50 \mu \mathrm{M}$ peptide treatment for $1 \mathrm{hr}$ at room temperature. Data bars represent means $\pm \mathrm{SEMs}$, $n=3$. Results are representative of 3 independent trials. 


\subsubsection{Safety assessment of peptide administration in vivo}

Based on results from the in vitro survival assays, we then tested the efficacy of peptide pre-treatment on C. rodentium infection in C57BL/6 mice. First, we evaluated safety of the peptide and placebo administration in vivo. Results showed that at the concentrations tested $(25-65 \mu \mathrm{M})$, overall health and well-being of the mice were not affected during the 10 day study. Additionally, crypt cell length of mice administered the peptide were not significantly different from that of mice administered matching concentrations of the placebo and sham-infected mice (Figure 2.11). These results suggest that peptide administration is safe in vivo and does not cause any adverse effects on mice.
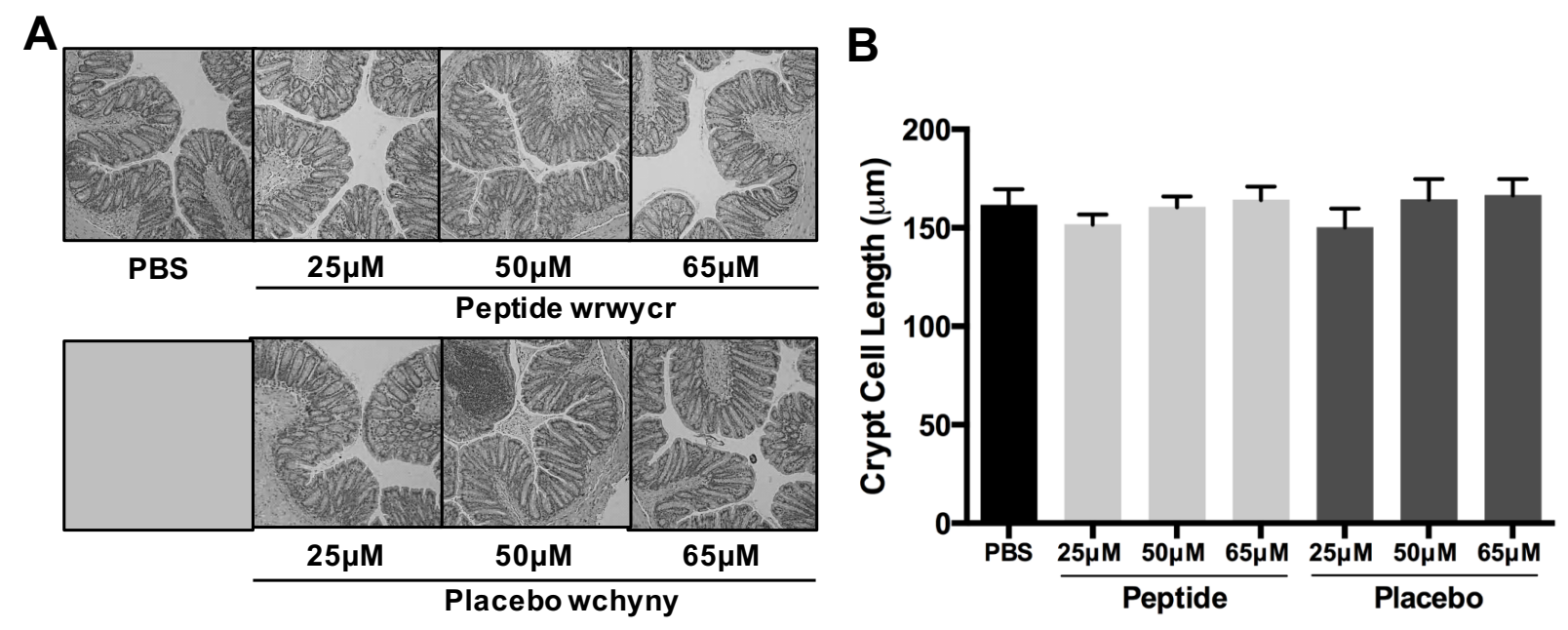

Figure 2.11: Safety testing of peptide administration in vivo. (a) Colonic epithelial cell hyperplasia in mice $10 \mathrm{~d}$ post-adminstration of peptide wrwycr or placebo wkhyny compared to PBS control. Representative micrographs of distal colon sections stained with hematoxylin-and-eosin stain. Black lines indicate crypt cell length. (b) Quantitative analysis of crypt cell lengths from b. Data bars represent means \pm SEMs (3 sections per animal, 10 counts per section). 


\subsubsection{Peptide pre-treatment of $C$. rodentium ameliorates infection and reduces infection outcomes in mouse infection model}

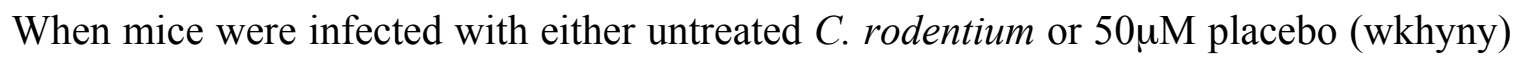
pre-treated-C. rodentium, there was $100 \%$ infection as assessed by fecal swab scores (Figure 2.12a). In contrast, mice infected with $50 \mu \mathrm{M}$ peptide pre-treated- $C$. rodentium showed no gut colonization resulting in $0 \%$ infection. There were no detectable fecal counts on MacConkey agar in peptide pre-treated-C. rodentium infected mice and no visible signs of illness, unlike the untreated and placebo pre-treated-C. rodentium infected mice where fecal counts were high and mice were visibly ill with hunched posture, fur ruffling, lethargy, and weight loss. Mice pre-treated with peptide and then challenged with $C$. rodentium were indistinguishable from sham, peptide, or placebo treated mice in terms of both general visual health and fecal counts on MacConkey agar. Similarly, qPCR analysis of espB expression in stool samples demonstrated the presence of $C$. rodentium at $1-2 \%$ of the total Eubacteria in the $C$. rodentium and placebo pre-treated-C. rodentium infected mice (Figure $\mathbf{2 . 1 2 b}$ ). This was significantly increased from the $0 \% C$. rodentium present in the peptide pre-treated-C. rodentium and uninfected control groups. Additional in vivo studies demonstrated that infection was peptide-dose-dependent, with $100 \%$ infection at the lower dose of peptide pre-treatment $(25 \mu \mathrm{M})$ and $25 \%$ of animals showing colonization at the higher dose of peptide pre-treatment $(65 \mu \mathrm{M})$ (Table 2.2).

Colonic epithelial cell hyperplasia, a characteristic feature of $C$. rodentium infection, was measured in haematoxylin-and-eosin-stained sections of distal colon (representative images are shown in Figure 2.12c). As expected, crypt cell length of C. rodentium-infected mice was significantly higher than in sham-challenged mice $(P<0.05)$ (Figure 2.12d). Similarly, crypt cell length of placebo pre-treated $C$. rodentium-infected mice was significantly higher than sham 
infected mice $(P<0.05)$. In contrast, crypt hyperplasia of peptide pre-treated-C. rodentium infected mice was not significantly different than mice challenged with peptide alone or the sham infected group $(P>0.05)$, indicating that colonic epithelial cell hyperplasia of $C$. rodentiuminfected mice was attenuated by pre-treatment with the peptide. Taken together, these results provide convincing evidence regarding the efficacy of peptide wrwycr in preventing C.rodentiuminduced colonic injury in a mouse model.

A

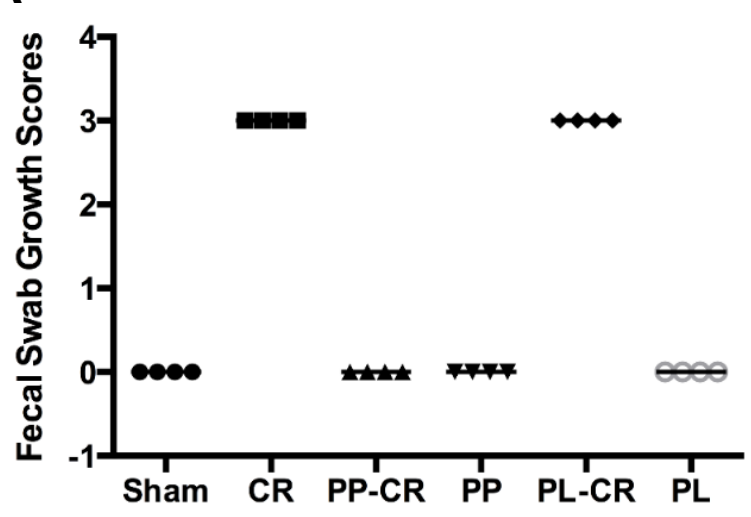

B

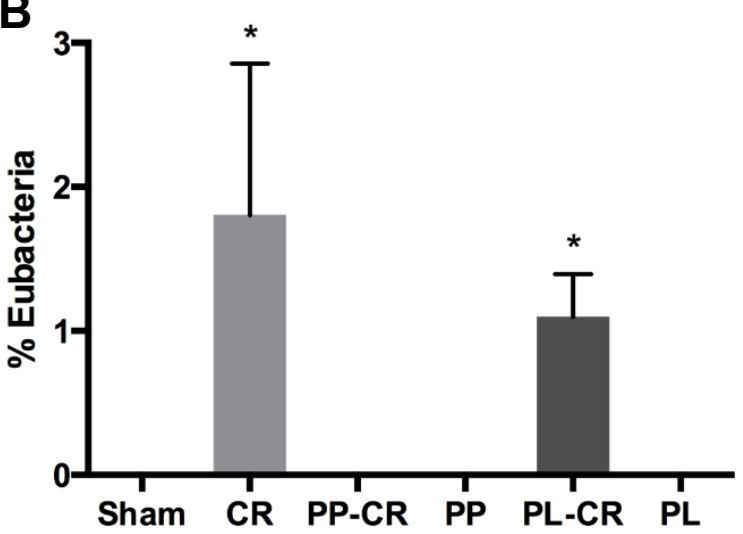

C

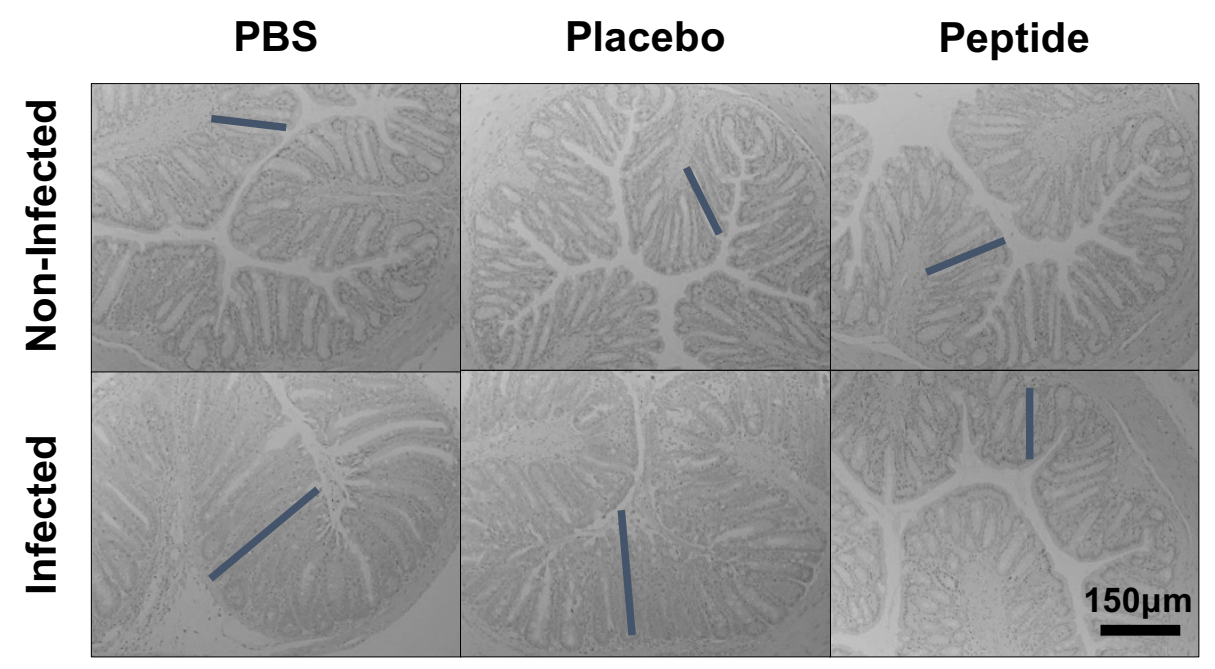




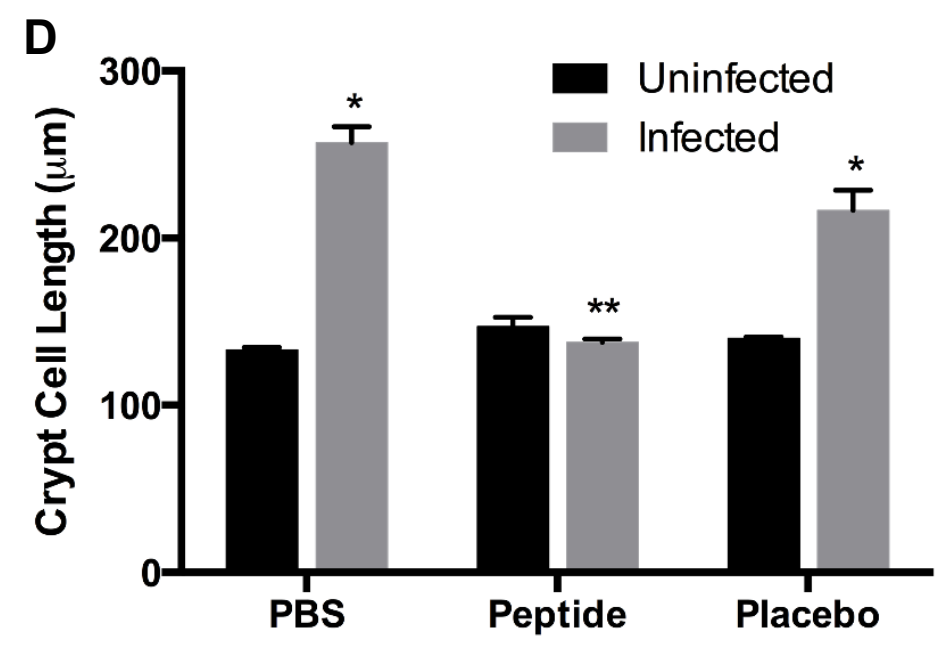

Figure 2.12: In vivo testing of peptide pre-treatment on $C$. rodentium infection. (a) Fecal swab growth scores of treated mice taken 10 days post-infection on MacConkey agar ( $n=4$ mice per study group). Sham - PBS, CR - C. rodentium, PP-CR - peptide pre-treated C. rodentium, PP - peptide alone, PL-CR - placebo pre-treated $C$. rodentium, PL- placebo alone. Colonies were scored based on a growth scale as previously described (Cantey and Hosterman, 1979), where $0=$ no growth, $1=$ widely spread colonies, $2=$ closely spaced colonies, and 3 = confluent growth. (b) Quantitative PCR analysis of EspB expression in stool samples taken $10 \mathrm{~d}$ post-infection $(n=4)$. Data bars represent means \pm SEMs. * indicates a significant difference from the peptide pre-treated $C$. rodentium and sham infected groups, $P<0.05$ by Mann-Whitney test. (c) Colonic epithelial cell hyperplasia in mice $10 \mathrm{~d}$ post-infection with $C$. rodentium $\left(10^{8} \mathrm{CFU}\right)$. Representative micrographs of distal colon sections stained with hematoxylin-and-eosin stain. Black lines indicate crypt cell length. (d) Quantitative analysis of crypt cell lengths from b. Data bars represent means \pm SEMs (3 sections per animal, 10 counts per section). * indicates a significant difference from non-infected controls, $P<0.05$ by two-way ANOVA. $* *$ indicates a significant difference from $C$. rodentium infected group, $P<0.05$ by two-way ANOVA. 
Table 2.2: Peptide pre-treatment prior to orogastric gavage prevents infection in mice infected with $C$. rodentium compared to uninfected mice in a dose-dependent manner.

\begin{tabular}{ccccccc}
\hline & \% Infection in Treatment Groups* \\
C. rodentium & $\ddagger$ & \multicolumn{3}{c}{ Peptide wrwycr pre-treated } & \multicolumn{2}{c}{ Placebo whhyny pre-treated } \\
& \multicolumn{3}{c}{ C. rodentium } & \multicolumn{3}{c}{ C. rodentium } \\
\cline { 2 - 7 } & $\mathbf{2 5 \mu M}$ & $\mathbf{5 0 \mu M}$ & $\mathbf{6 5 \mu M}$ & $\mathbf{2 5 \mu M}$ & $\mathbf{5 0 \mu M}$ & $\mathbf{6 5 \mu M}$ \\
\hline $92 \%$ & $100 \%$ & $0 \%$ & $25 \%$ & $100 \%$ & $100 \%$ & $100 \%$ \\
\hline
\end{tabular}

Fecal swabs obtained from mice at Day 10 post-infection were plated onto MacConkey Agar and incubated overnight at $37^{\circ} \mathrm{C}$ ( $n=4$ mice per group; ${ }^{\ddagger} n=12$ mice). Plates with colony growth were counted as infected. *All uninfected control treatment groups had $0 \%$ infection ( $n=4-12$ mice per group).

\subsubsection{Peptide pre-treatment reduces $C$, rodentium-induced alterations of the gut microbiome}

Fecal microbial composition analysis of the indicated $C$. rodentium-infected mice demonstrated $C$. rodentium-induced alterations in the gut microbiome; namely a significant increase in Gammaproteobacteria and a decrease in Lactobacillus (Figure 2.13a-c). Similar results were also seen for the placebo wkhyny pre-treated- $C$. rodentium infected group. In contrast, when $C$. rodentium was pre-treated with peptide wrwycr prior to infection, there was a significant reduction in $C$. rodentium-induced alterations of the fecal microbial composition. Interestingly, when mice were treated with peptide wrwycr alone, there was no significant alteration of the ratio of Bacteriodetes:Firmicutes, nor in the levels of Lactobacillus, or Gammaproteobacteria compared to the sham-infected group (Figure 2.13a-c). Taken together, these results confirm that peptide pre-treatment protects against $C$. rodentium-induced alterations of the gut microbiome in vivo, and that peptide administration alone does not alter the gut microbial composition. 
A
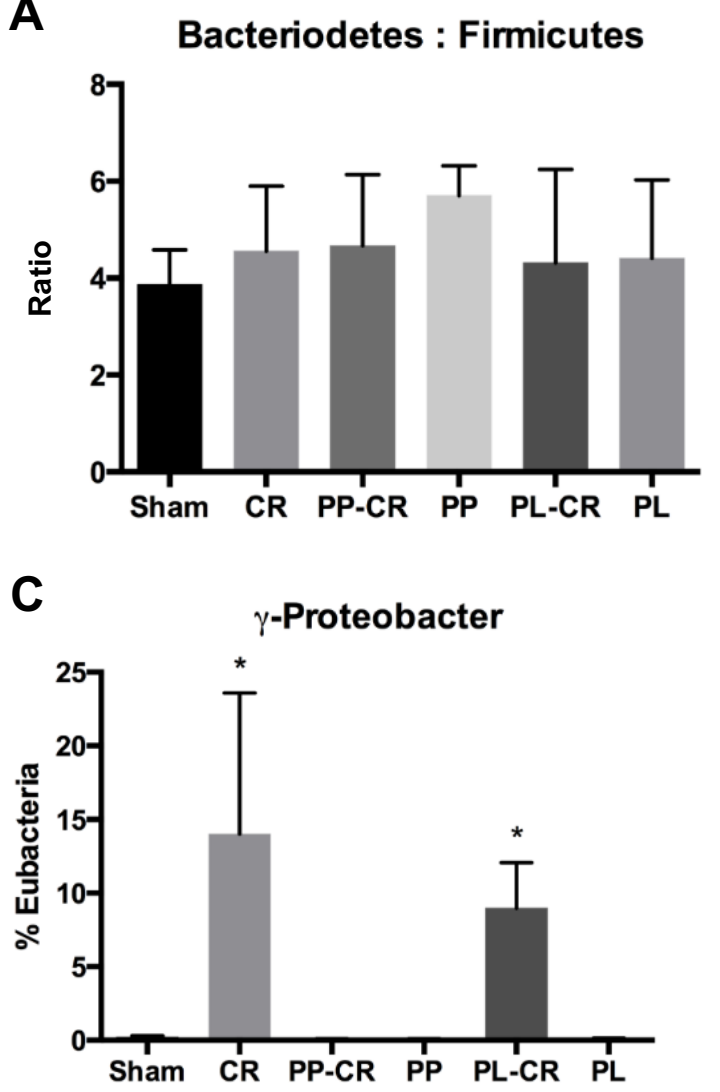

B

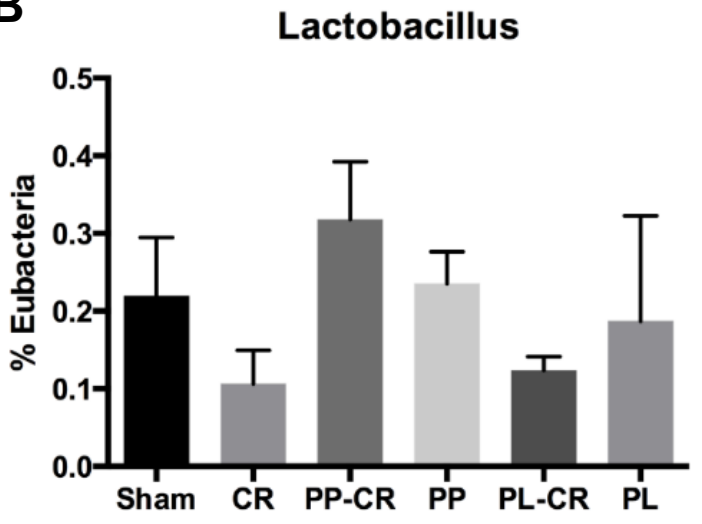

Figure 2.13: qPCR analysis of fecal microbial composition of treated mice $10 \mathrm{~d}$ post-infection. Semiquantitative amount of (a) Bacteriodetes:Firmicutes; (b) Gammaproteobacteria; and (c) Lactobacillus relative to total Eubacteria. Data bars represent means \pm SEMs ( $n=4$ mice per study group). * indicates a significant difference from sham and peptide pre-treated-C. rodentium infected mice, $P<0.05$ by MannWhitney test.

\subsection{DISCUSSION}

This is the first study to date that demonstrates the efficacy of the antimicrobial peptide wrwycr as a prevention strategy in reducing disease outcome in a $C$. rodentium murine model of EHEC O157:H7 infection. Both the in vitro and in vivo data presented here indicate that a brief pre-treatment phase of $C$. rodentium with wrwycr followed by exposure to acute acid stress, 
representative of gastric passage, dramatically reduced bacterial counts to below the infectious dose of $10^{7} \mathrm{CFU}$, thereby decreasing the potential for successful colonization and subsequent infection to occur.

The in vitro data clearly demonstrate that while peptide or acid treatment alone can increase C. rodentium killing, it is the combination of peptide pre-treatment followed by acid treatment that dramatically reduces survival to below the infectious dose. Previous research has shown that the murine gastric fluid ranges in $\mathrm{pH}$ from 3.0 to 4.0, depending on the state of feeding, which is less acidic than the human counterpart which has a pH of approximately 2.0 (Brenneman et al., 2014; Richard and Foster, 2004; Smith, 2003). These $\mathrm{pH}$ values are acidic enough to induce significant levels of DNA damage, thereby allowing the peptide wrwycr to inhibit DNA repair by outcompeting RecG and RuvC during HJ resolution. This inhibition of DNA repair, in turn, leads to enhanced levels of bacterial cell death due to the DNA damage inflicted by acute acid stress. Additionally, the exposure to acid stress may be synergistic with the peptide's effect on the cell membrane and the cell's inability to maintain sufficient levels of bioavailable iron, resulting in increased toxicity to $C$. rodentium. Regardless of its exact mechanism, these results demonstrate that the combination of peptide treatment with acid stress is critical in maximizing the effect of the peptide on inhibition of DNA repair. Consequently, this finding underscores the fact that, at the low does used in this study, the peptide would be less effective as a post-infection treatment due to the absence of acute acid stress in the distal colon.

The combination of peptide-acid treatment was shown to be most effective in enhancing bacterial cell death of planktonic $C$. rodentium, while remaining relatively ineffective against established C. rodentium biofilms. Bacterial biofilms have been shown to display increased resistance to many antimicrobial agents, using a combination of physical and chemical barriers to 
prevent antimicrobial penetration into the biofilm (Mah and O'Toole, 2001). While this may explain why an increase in bacterial cell death was not observed in C. rodentium biofilms following peptide treatment, there are likely other mechanisms in place which cause resistance to peptide treatment. These alternative mechanisms may include slow growth, transition of biofilm cells into a resistance phenotype, and alterations of the microenvironment within the biofilm (Mah and O’Toole, 2001; Stewart and Costerton, 2001).

A vital aspect to any study of antimicrobials is the ability of the pathogen to recover from sub-inhibitory concentrations of the antimicrobial agent. The findings of this study showed that prolonged treatment of $C$. rodentium with the peptide alone resulted in either partial or full growth recovery only after $50 \mathrm{hrs}$, and that recovery was dose-dependent with the highest concentrations of peptide showing lower levels of recovery. The growth recovery seen may be attributed to the peptide being consumed as greater numbers of bacteria accumulate in the culture through cell division. Alternatively, the recovery of bacterial growth may also be due to the employment of various antimicrobial resistance mechanisms by the pathogen, such as upregulation of efflux systems that prevent the drug from reaching its intracellular target, or production of alternative metabolic pathways that bypass the action of the antimicrobial drug (McManus, 1997; Tenover, 2006). In contrast, when peptide pre-treatment was followed by acid exposure, recovery was significantly impaired, with no growth recovery seen at the highest peptide concentration tested and relatively limited recovery at the lower concentrations. These observations indicate that while C. rodentium can recover from peptide treatment alone, the ability to recover is strongly compromised when peptide pre-treatment is followed by acute acid stress as revealed by both the in vitro and in vivo studies. Moreover, when C. rodentium is treated with peptide for a $24 \mathrm{hr}$ period then exposed to a second treatment of peptide, they are at least equally if not more sensitive to the 
second dose of peptide, with no evidence of resistance in the recovered population. These results suggest that a second course of peptide treatment can mitigate recovery after the first peptide treatment, although multiple exposures to peptide treatment may induce resistance to further peptide treatment.

Finally, the in vivo studies confirmed that administration of peptide over the range of concentrations tested in this study caused no adverse effects on the overall health and well-being of mice over the 10 day study period. Additionally, colonic histology analysis of peptide-treated mice was unchanged relative to the sham and placebo-treated groups. These findings are consistent with previous reports that show no evidence of cytotoxicity in a variety of human cell lines and

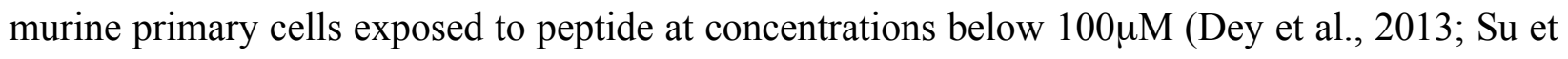
al., 2010). Moreover the in vivo studies revealed that $50-65 \mu \mathrm{M}$ peptide pre-treatment of $C$. rodentium prior to infection of mice ameliorated any evidence of infection based on C. rodentium colonization levels, fecal scores, colonic histology, and visual observation of overall animal health. Furthermore, analysis of the fecal microbiome showed no differences between the peptide pretreated $C$. rodentium infected group and the sham infected group, while there was a significant difference between the peptide pre-treated $C$. rodentium infected group and the $C$. rodentium infected and placebo pre-treated C. rodentium infected groups. These findings provide further evidence that peptide pre-treatment of $C$. rodentium prior to infection is effective at preventing infection in vivo.

In summary, both the in vitro and in vivo findings of this study confirm and extend our previous findings which show a dose-dependent increase in acid-induced killing of peptide pretreated EHEC seropathotypes associated with severe human disease (Lino et al., 2011). 


\subsection{CONCLUSIONS \& FUTURE DIRECTIONS}

\subsubsection{Conclusions}

In summary, we have successfully demonstrated that peptide wrwycr pre-treatment of $C$. rodentium followed by acute acid stress, representative of passage through gastric acid, is effective at significantly reduced pathogen survival in vitro and ameliorating infection in a murine model of EHEC O157:H7 infection. The efficacy of peptide-acid induced pathogen killing was seen regardless of bacterial growth phase, and only low levels of growth recovery were observed up to 72 hours later in vitro. Additionally, when C. rodentium was pre-treated with peptide prior to infection of mice, the infection outcomes such as colonic histology and fecal microbiota composition were unchanged compared to the sham-challenged mice. Furthermore, peptide administration alone did not affect fecal microbiota composition or colonic histology, indicating that these markers of colonic health were unaffected at the peptide doses tested. Taken together, these findings support a potential role for the peptide wrwycr as a prevention strategy against enteric pathogen infection by enhancing the body's natural defense system of gastric acid to compromise bacterial survival prior to intestinal colonization. In the proposed model (Figure 2.14), foods such as beef and spinach that are commonly associated with EHEC outbreaks would be pre-treated with the peptide at the grocery store level as a means of getting the peptide into the pathogen. Once ingested by the host, the contaminated food enters the stomach where the low $\mathrm{pH}$ of gastric acid induces a high amount of bacterial DNA damage. As the bacteria attempt to repair this DNA damage, HJs are formed as an intermediate structure in the repair process and their subsequent resolution is inhibited by peptide wrwycr. This inhibition of DNA repair, together with the peptide's additional effects on iron toxicity within the cell, results in a dramatic increase in bacterial cell death prior to entering the small intestine. Since no viable bacterial cells are able to 
enter the small intestine, they are therefore unable to colonize the host at the preferred site of colonization in the large intestine.

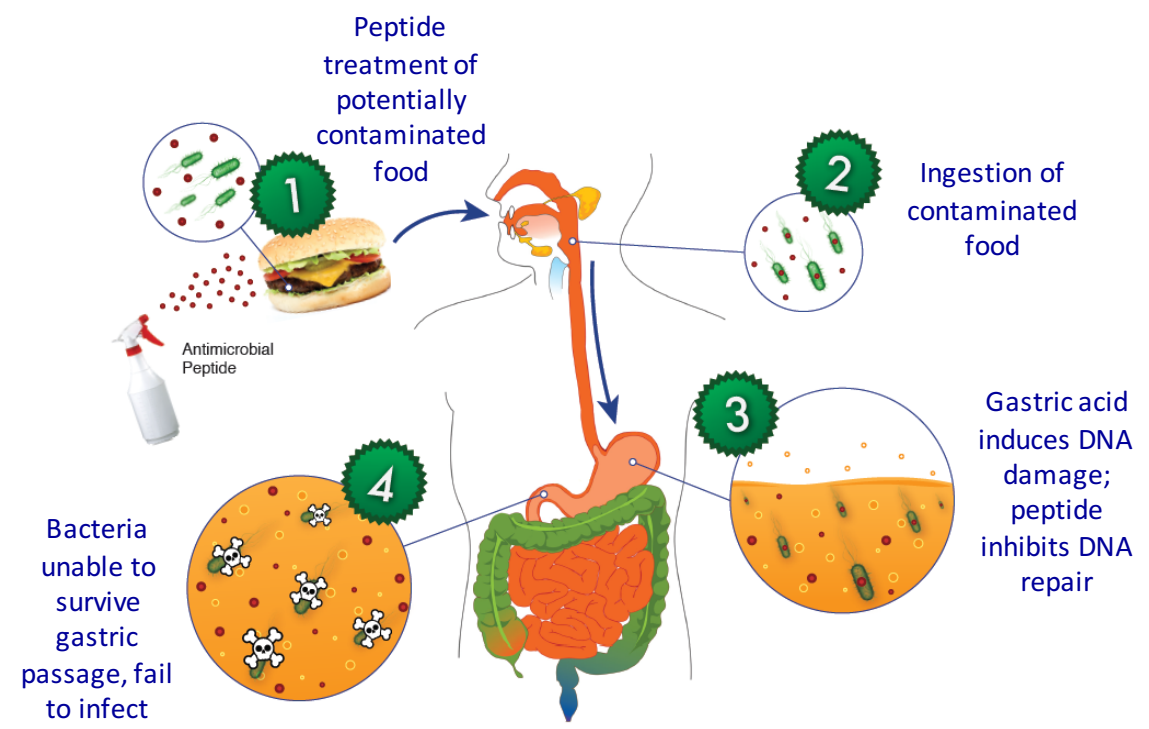

Figure 2.14: Model of the antimicrobial peptide wrwycr as a prevention strategy for EHEC infection. In the proposed model, commonly contaminated foods are pre-treated with peptide wrwycr. Once ingested, the ingested food then enters the stomach where the low $\mathrm{pH}$ exposure induces significant DNA damage. During the DNA repair process, the resolution of HJs is inhibited by the antimicrobial peptide thereby inhibiting DNA repair and causing bacterial cell death.

While this model provides promise as a prevention strategy for EHEC infection, there are still many challenges that need to be examined. For example, questions that need to be addressed include the stability of the peptide, required frequency of antimicrobial treatment of foods, the optimal temperature for peptide uptake, the potential for development of peptide resistance after multiple treatments, and the safety of multiple ingestions of peptide-treated foods by the host. Addressing these and other key questions represents an important next step. 


\subsubsection{Future Directions \& Significance}

While this study successfully demonstrates the use of this antimicrobial peptide as an effective prevention strategy using an in vivo animal model, there is still much to be investigated. When considering the use of this peptide as a therapeutic, it is important to note that the minimum effective dose of peptide wrwycr tested in vivo is $50 \mu \mathrm{M}$. While the use of this peptide at $50 \mu \mathrm{M}$ has been demonstrated to be safe to the host, its use as a widespread preventative strategy can be limiting due to high costs of using such a high concentration. An alternative, and more costeffective, approach would be to combine the use of this peptide with a second antimicrobial that acts in a similar manner at a lower effective concentration. This dual antimicrobial approach may prove to be even more potent than the use of peptide wrwycr on its own while being cost-effective. Additionally, the combination of peptide wrwycr treatment with a second antimicrobial that induces acid stress, DNA damage, or oxidative stress would enhance the efficacy of peptide wrwycr treatment and may enable it to be used as a post-infection treatment as well as a prevention strategy. Secondly, a vital aspect of the proposed model involves the inclusion of the peptide in foods that are at high risk of contamination. To better test this model, an in vivo model in which the animal chow is exposed to $C$. rodentium or peptide pre-treated $C$. rodentium prior to ingestion by the animal may be useful. A final aspect of the peptide's efficacy that would be beneficial for marketing of it as an antimicrobial strategy would be testing of its potency on other foodborne pathogens such as Salmonella, Listeria, Clostridium botulinum, Campylobacter, and Vibrio vulnificus. Since the mechanisms by which RecG and RuvC promote HJ resolution are conserved in over 300 bacterial species, it is likely that peptide wrwycr treatment will act in a similar manner as seen in EHEC in these other foodborne pathogens. If the peptide is able to act as an antimicrobial in the majority of foodborne enteric pathogens, its marketability would significantly increase. 
Interestingly, the combination of peptide treatment and acid stress represents a significant advancement in the development of preventative strategies for EHEC infection, as this strategy not only introduces a new antimicrobial but utilizes an antimicrobial to enhance the host innate defense systems. The key to this novel and innovate strategy is to kill the pathogen prior to colonization of the host by enhancing the killing action of the innate host defenses. This novel strategy has widespread potential as it can be utilized to potentiate the pathogen killing action of such host defenses as bile salts, CAMPs, and SCFAs.

In conclusion, the use of this novel antimicrobial strategy has widespread potential as an antimicrobial prevention strategy which targets EHEC, and potentially other foodborne pathogens, prior to colonization of the host, thereby eliminating the adverse effects caused by Shiga toxin secretion into the bloodstream. Moreover, if the studies testing a combination of peptide wrwycr with another antimicrobial compound proves successful, it may also show potential as a postinfection treatment in addition to its use as a preventative strategy. This research represents a major breakthrough in the treatment, and more specifically prevention, of EHEC infection which may lead to a reduction in the outbreaks seen globally each year by enhancing the innate human defense system through the use of small antimicrobial peptides. 


\section{Chapter 3: Examining the Role of DNA-Binding Protein, Dps, in EHEC's Response to Peptide wrwycr Treatment}

Lackraj, T., Kacori, M., \& Foster, D.B. Dps protects Enterohemorrhagic Escherichia coli against acid-induced antimicrobial peptide killing. [Manuscript in preparation] 


\subsection{ABSTRACT}

Dps, a DNA-binding protein from starved cells in E. coli, is a member of the ferritin superfamily and is part of a bacterial defense system that protects DNA against damage. It is also involved in protection against cellular stresses such as UV and gamma irradiation, iron and copper toxicity, thermal stress, and acid and base stress. We previously demonstrated that a novel antimicrobial peptide wrwycr enhances acid-induced killing of enterohemorrhagic Escherichia coli (EHEC) and ablates infection in a C. rodentium mouse model of EHEC infection. Wrwycr has been shown to bind to Holliday Junctions and prevent their resolution. It has also been shown to increase chelatable iron within the cell, suggesting that it may promote the formation of hydroxyl radicals through the Fenton Reaction. This, combined with the effect of peptide and acid stress on DNA damage, suggests a key defense role for Dps in peptide- and peptide-acid induced killing of EHEC. The goal of this study is to evaluate the role of Dps in peptide- and peptide-acid induced killing of EHEC. Our results demonstrate that disruption of the dps gene in EHEC O157:H7 enhances each of acid-, or peptide-, or peptide-acid-induced killing relative to the wild-type EHEC, and complementation restores it. Using flow cytometric analysis, we have also demonstrated increased levels of hydroxyl radicals in peptide-treated wild-type EHEC relative to the untreated control. Disruption of the $d p s$ gene further increases hydroxyl radical levels. These findings indicate that peptide treatment of EHEC enhances the formation of hydroxyl radicals, likely through the Fenton reaction thereby contributing to the killing action of the peptide, and that $d p s$ protects against peptide killing of EHEC, in part by protecting against hydroxyl radical formation. We have also demonstrated through survival assays and flow cytometry of hydroxyl radical formation that the peptide wrwycr acts similarly to the bactericidal class of antibiotics, supporting a role for this novel antimicrobial peptide as a prevention strategy against EHEC infection. This 
study provides important insights into antimicrobial peptide wrwycr-mediated killing of EHEC, which could be exploited in the development of more effective antimicrobials.

\subsection{INTRODUCTION}

\subsubsection{Bactericidal Antibiotics}

Bactericidal antibiotics are a class of drugs that kill bacteria with an efficiency of greater than $99.9 \%$, while bacteriostatic drugs do not kill bacteria but rather simply inhibit growth (Kohanski et al., 2007; Pankey and Sabath, 2004). The novel antimicrobial peptide, wrwycr, was previously shown to cause an increase in bacterial killing, similarly to that seen by bactericidal antibiotics, suggesting that it may behave in a manner parallel to this class of drugs. The drugtarget interactions of these bactericidal antibiotics are predominantly classified into 3 categories: beta lactams that inhibit cell wall biosynthesis, fluoroquinolones that inhibit DNA replication and repair, and aminoglycosides that inhibit protein synthesis through interference with proper ribosome function (Dwyer et al., 2015; Kohanski et al., 2007; Von Döhren, 2009; Walsh, 2000). Interestingly, bactericidal antibiotics, unlike bacteriostatic antibiotics, stimulate the production of hydroxyl radicals in both gram negative and gram positive bacteria alike (Kohanski et al., 2007). More specifically, bactericidal antibiotics cause an increase in $\mathrm{Fe}^{2+}$ within the bacterial cell, likely due to the breakdown of iron-sulfur clusters, which drives the Fenton reaction forward, thereby leading to the formation of highly deleterious hydroxyl radicals which eventually result in bacterial cell death (Figure 3.1) (Kohanski et al., 2007). In contrast, the bacterial cell can be protected against hydroxyl radical-induced cell death through the expression of DNA-protecting proteins such as Dps. 


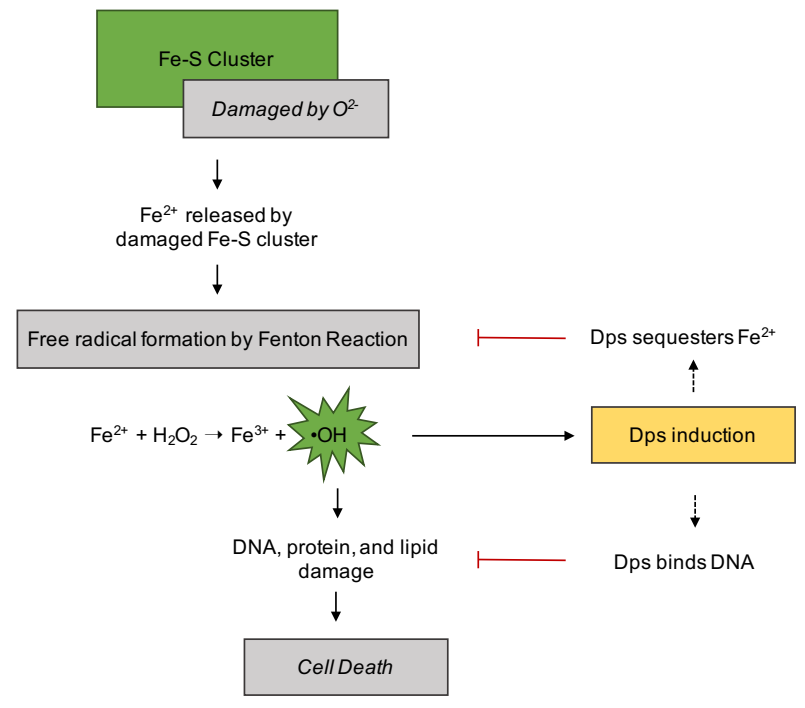

Figure 3.1: Bactericidal antibiotics mode of action. The breakdown of iron-sulfur clusters leads to the release of $\mathrm{Fe}^{2+}$ and formation of hydroxyl radicals through Fenton Chemistry, thereby resulting in cell death. This can be mediated through expression of DNA-protecting proteins such as Dps.

\subsubsection{Dps, a DNA-Binding Protein}

\subsubsection{Dps as a Protective and Regulatory Protein}

The E. coli DNA-binding protein from starved cells (Dps) belongs to a subfamily of ferritin-like proteins that was first characterized as a DNA-binding protein induced during stationary phase or in cells under stress (Almirón et al., 1992; Huergo et al., 2013). Dps proteins are widely distributed in the bacterial kingdom, with the sequence being conserved in over 300 bacterial species. Bacterial genomes typically encode one Dps protein, although some may encode up to two or three (Chiancone and Ceci, 2010; Karas et al., 2015). E. coli Dps shares many structural features in common with other bacterial ferritins and is composed of at least 12 subunits that form spherical dodecamers (Choi et al., 2000; Grant et al., 1998; Wolf et al., 1999). Dps expression is seen at low levels during late exponential growth and slowly accumulates during the stationary growth phase until it becomes the most abundant protein in the cell (Ali Azam et al., 
1999; Halsey et al., 2004; Jeong et al., 2008). Moreover, E. coli Dps expression is regulated by the stationary phase sigma factor RpoS $\left(\sigma^{38}\right)$, OxyR, and IHF (Altuvia et al., 1994; Halsey et al., 2004).

Since bacteria encounter a multitude of stresses in the environment, within foods and during food processing, and throughout the human gastrointestinal tract, they have evolved a variety of strategies to survive and adapt under such stressful conditions. These strategies range from measures such as sporulation to the expression of stress mediation proteins such as Dps (Battesti et al., 2011; Karas et al., 2015). E. coli Dps acts as a critical component of various stress response pathways in bacterial cells and enhances survival during exposure to a multitude of stresses such as starvation, heat shock, oxidative stress, UV and ionizing radiation, acid and base stress, and metal toxicity (Almirón et al., 1992; Altuvia et al., 1994; Jeong et al., 2008; Martinez and Kolter, 1997; Nair and Finkel, 2004). These protective effects of Dps are likely a result of either one or both of its dual biochemical functions - DNA binding and ferroxidase activity (Calhoun and Kwon, 2011; Karas et al., 2015).

\subsubsection{DNA-Binding as a Protection Mechanism}

The physical mode of protection by Dps, DNA binding, is not common to all members of the Dps family. In the bacterial species where Dps-DNA complexes are formed, they play a vital protective role against such stresses as acid and base shock, iron and copper toxicity, high salt or radiation, and oxidative stress (Chiancone and Ceci, 2010; Nair and Finkel, 2004). While DNA is the common target for Dps protection through physical association, Dps-DNA binding has no apparent sequence specificity although the complex proves to be extremely stable and thereby resistant to DNase and oxidative damage (Almirón et al., 1992; Chiancone and Ceci, 2010; Jeong et al., 2008). The DNA binding activity of Dps is activated by $\mathrm{Fe}^{2+}$ or $\mathrm{H}_{2} \mathrm{O}_{2}$ at acidic $\mathrm{pH}$, while 
binding is inhibited by $\mathrm{NaCl}$ and $\mathrm{Mg}^{2+}$ which suggests the role of ionic interactions between Dps and DNA (Huergo et al., 2013). The exact mechanism through which Dps binds DNA is poorly understood, although it has been suggested that an initial DNA binding event occurs prior to condensation into highly ordered DNA-protein structures (Ceci et al., 2007; Chiancone and Ceci, 2010).

The DNA shielding provided through Dps-DNA binding provides a major mode of protection for the bacterial cell against the chemical and reactive damage caused during exposure to acid and oxidative stress (Calhoun and Kwon, 2011; Zhao et al., 2002). Interestingly, in vitro studies have confirmed that Dps-DNA binding protects DNA from low $\mathrm{pH}$-induced damage. Moreover, the ability of Dps-bound DNA to condense into a co-crystallized structure protects the DNA against acid stress by maintaining genetic integrity (Calhoun and Kwon, 2011; Jeong et al., 2008). Together these findings confirm a vital role for Dps in acid tolerance and oxidative stress protection in E. coli (Choi et al., 2000).

\subsubsection{Ferroxidase Activity as a Protection Mechanism}

In addition to its physical mode of protection through DNA binding, Dps also exhibits a chemical mode of protection through its ferroxidase activity. Unlike other members of the ferritin superfamily, the ferroxidase activity of Dps prefers $\mathrm{H}_{2} \mathrm{O}_{2}$ over $\mathrm{O}_{2}$ when carrying out iron oxidation (Chiancone and Ceci, 2010; Franceschini et al., 2006; Zhao et al., 2002). The most distinct structure of the Dps family is the ferroxidase center, which is located between the subunits where two $\mathrm{Fe}^{2+}$ ions bind to form a binuclear iron center (Ilari et al., 2000; Zeth, 2012). Through the ferroxidase activity of Dps, DNA protection is exerted indirectly via the removal of free $\mathrm{Fe}^{2+}$ from the cytoplasm which in turn reduces the formation of highly reactive oxygen species (hROS) 
through Fenton chemistry (Almirón et al., 1992; Ceci et al., 2007; Huergo et al., 2013). This is a critical component of E. coli's protective mechanism as excess iron within the cell can become toxic and potentially fatal through the production of hROS. However, once iron is bound within the ferroxidase center of Dps, it is oxidized from ferrous ions to ferric ions which are non-toxic to the cell (Calhoun and Kwon, 2011; Nair and Finkel, 2004). Together, these findings demonstrate a vital role for Dps in protection against iron toxicity in E. coli cells.

\subsubsection{Rationale and Overall Aim}

While the effects of peptide wrwycr treatment on bacterial cells have been previously studied, the relationship between its different roles in interfering with DNA damage repair and restricting iron availability within the cell is poorly understood (Orchard et al., 2012). The DNA binding protein, Dps, represents an interesting DNA-protecting mechanism which involves protection against multiple cellular stresses including acid stress and iron toxicity. Consequently, Dps may provide a unique link between the different effects of peptide treatment. It is hypothesized that Dps expression may protect EHEC against peptide wrwycr and peptide-acid treatment both through (1) binding DNA to protect against the detrimental effects of peptide-acid treatment, and (2) sequestering $\mathrm{Fe}^{2+}$ to protect against iron toxicity that leads to hydroxyl radical formation. Furthermore, it is hypothesized that peptide wrwycr behaves as the bactericidal class of antibiotics by causing bacterial killing and inducing hydroxyl radical formation. The overall objective of this research project then is to determine the role of Dps as a protective mechanism during acid, peptide, and peptide-acid treatment of EHEC through its DNA-binding and ferroxidase center abilities, as well as to confirm whether peptide wrwycr falls into the bactericidal classification of antibiotic drugs. 


\subsection{MATERIALS AND METHODS}

\subsubsection{Bacterial strains and culture conditions}

Escherichia coli $\mathrm{O} 157: \mathrm{H} 7$ strains used for this study (kindly provided by Dr. Charles Kaspar, University of Wisconsin, Madison, WI) are listed in Table 3.1. Bacterial glycerol stocks were maintained at $-80^{\circ} \mathrm{C}$, and were streaked onto LB (BioShop, Burlington, ON, Canada) agar containing the appropriate antibiotics prior to use in order to obtain single colonies. Overnight cultures were prepared by inoculating single colonies into LB broth containing the appropriate antibiotics and then incubating $12-16$ hours at $37^{\circ} \mathrm{C}$ with shaking.

Table 3.1: Bacterial strains and plasmids used in study.

\begin{tabular}{lll}
\hline Strain & Relevant Characteristics & Reference \\
\hline EDL933, ATCC 43895 & $\begin{array}{l}s t x_{1} \text { stx }_{2} \text {; serotype O157:H7 isolate from } \\
\text { hamburger }\end{array}$ & (Choi et al., 2000) \\
\hline EDL933 $d p s:: n p t I$ & $\mathrm{Km}^{\mathrm{r}}$ & (Choi et al., 2000) \\
\hline EDL933 $d p s:: n p t I / \mathrm{pUC18}+d p s$ & $\mathrm{Km}^{\mathrm{r}}, \mathrm{Ap}^{\mathrm{r}}$ & This study \\
\hline EDL933 $d p s:: n p t I / \mathrm{pUC18}$ & $\mathrm{Km}^{\mathrm{r}}, \mathrm{Ap}^{\mathrm{r}}$ & This study \\
\hline
\end{tabular}

\subsubsection{Complementation of the $d p s:$ :npt1 mutant}

The $d p s$ gene and upstream promoter region was amplified from EHEC O157:H7 genomic DNA by PCR using a pair of primers carrying EcoRI or PstI sites on the 5' ends: (1) 5'CGGAATTCCATAACCATGCAGAATTTCT-3' and (2) 5'-CGGCTGAGCAGCGATGGATTT ATTCGAT-3'. The resulting PCR fragment was digested with EcoRI and Pst (FastDigest Enzymes; ThermoFisher, Scientific, Waltham, MA, USA), and ligated into the multiple cloning site of the vector pUC18, previously digested with the same enzymes. The resulting plasmid, pUC18 with $d p s$, was analyzed for insertion of the PCR fragment using PCR amplification of the 
$d p s$ gene and gene sequencing using $d p s$-specific primers (ACGT Corp, Toronto, ON, Canada). Sequence comparisons were conducted using Serial Cloner 2.1.

\subsubsection{Antimicrobial peptide wrwycr}

The peptide wrwycr was synthesized with a C-terminal amide group, purified to $>95 \%$ purity at Sigma-Genosys or Biosynthesis and dissolved in 50\% dimethyl sulphoxide (DMSO), as described previously (Gunderson and Segall, 2006). A wrwycr stock solution (10mM) was maintained at $-20^{\circ} \mathrm{C}$ in 50 or $100 \%$ DMSO.

\subsubsection{Bactericidal versus bacteriostatic antibiotic assay}

To compare the action of the peptide wrwycr to the bactericidal and bacteriostatic class of antibiotics, an overnight bacterial culture of EHEC EDL933 WT was diluted 1:25 in fresh LB broth and grown 1.5 hours at $37^{\circ} \mathrm{C}$ with shaking to mid-exponential phase $\left(\mathrm{OD}_{600} 0.4-0.6\right)$. Bacteria were pelleted at 3,500 rpm for $10 \mathrm{~min}$ at $4{ }^{\circ} \mathrm{C}$ and re-suspended in Mueller Hinton Broth

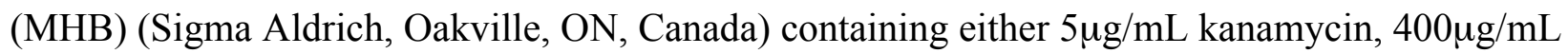
spectinomycin, or $50 \mu \mathrm{M}$ peptide wrwycr. Samples were incubated at $37^{\circ} \mathrm{C}, 300 \mathrm{rpm}$ for 3 hours. Samples were taken at $\mathrm{t}=0,1,2,3 \mathrm{hrs}$, serially diluted in $1 \mathrm{X}$ PBS, plated onto LB agar and grown overnight at $37^{\circ} \mathrm{C}$. Additional samples were taken at $\mathrm{t}=3 \mathrm{hr}$ and prepared for flow cytometric analysis of hydroxyl radical formation (see below).

\subsubsection{Survival assays}

To determine the role of Dps in acid stress of EHEC, overnight bacterial cultures of EHEC were diluted 1:25 (wild-type and $d p s^{-}$) or 1:15 (dps-/dps and $\left.d p s-/ p U C 18\right)$ in fresh LB broth and 
grown 2 hours at $37^{\circ} \mathrm{C}$ with shaking to early stationary phase $\left(\mathrm{OD}_{600} 0.9-1.0\right)$. Bacteria were pelleted at 3,500 rpm for $10 \mathrm{~min}$ at $4^{\circ} \mathrm{C}$, re-suspended in $1 \mathrm{X} \mathrm{PBS}$ at $\mathrm{pH} 3.5$, and then incubated at $37^{\circ} \mathrm{C}$ for 1 hour, static. Samples were taken at $\mathrm{t}=0,15,30,45$, and 60 mins, serially diluted in $1 \mathrm{X}$ PBS, plated onto LB agar and grown overnight at $37^{\circ} \mathrm{C}$.

In order to evaluate the role of Dps in peptide treatment of EHEC, overnight bacterial cultures of EHEC were subcultured as outlined above. Bacteria were pelleted at 3,500 rpm for 10 min at $4^{\circ} \mathrm{C}$, re-suspended in $0.5 \mathrm{X}$ PBS containing $50 \mu \mathrm{M}$ peptide wrwycr, and then incubated at room temperature for 1 hour, static. Samples were taken at $\mathrm{t}=0,15,30,45$, and 60 mins, serially diluted in 1X PBS, plated onto LB agar and grown overnight at $37^{\circ} \mathrm{C}$.

Finally, to assess the role of Dps in peptide-acid treatment of EHEC, overnight bacterial cultures of EHEC were subcultured as above. Bacteria were pelleted at 3,500 rpm for $10 \mathrm{~min}$ at $4^{\circ} \mathrm{C}$, re-suspended in $0.5 \mathrm{X}$ PBS containing $50 \mu \mathrm{M}$ peptide wrwycr, and then incubated at room temperature for 15 mins, static. Bacteria were pelleted as above, re-suspended in $1 \mathrm{X} \mathrm{PBS}$ at $\mathrm{pH}$ 3.5, and incubated at $37^{\circ} \mathrm{C}$ for 1 hour, static. Samples were taken at $\mathrm{t}=\mathrm{UT}$ (immediately prior to peptide addition), 0 (immediately prior to acid addition), 15, 30, 45, and 60 mins, serially diluted in 1X PBS, plated onto LB agar and grown overnight at $37^{\circ} \mathrm{C}$.

\subsubsection{Flow cytometric analysis of hydroxyl radical formation}

Bacterial cultures of EHEC were grown and subjected to either acid ( $\mathrm{pH} 3.0$ ), peptide wrwycr $(50 \mu \mathrm{M})$, or peptide-acid $(50 \mu \mathrm{M}-15 \mathrm{mins}, \mathrm{pH} 3.0-1 \mathrm{hr})$ stress as outlined above. Bacteria were pelleted as above, re-suspended in $2 \%$ PFA, and incubated at room temperature for 15 mins, static. Following fixation, bacteria were pelleted, washed 1X in PBS, and diluted 1:10 in 1X PBS,

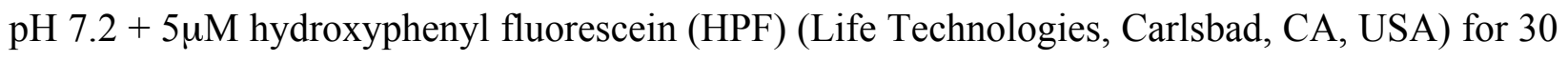


min at room temperature. Propidium iodide (Life Technologies, Carlsbad, CA, USA) was added to each sample just prior to measuring with a $488 \mathrm{~nm}$ argon laser on a Becton Dickinson LSR Fortessa X-20 Cell Analyzer. At least 10,000 cells were collected for each sample.

\subsubsection{Statistical analyses}

All data are represented as means \pm standard error of the means (SEMs) of at least 3 independent experiments. Either one-way or two-way analysis of variance (ANOVA) with Tukey's multiple-comparison test was used to determine differences among multiple groups. A $P$ value of $<0.05$ was considered significant.

\subsection{RESULTS}

\subsubsection{Complementation of the dps::nptI mutant}

Positive transformants from the cloning of the $d p s$ ORF and promoter from E. coli O157:H7 EDL933 into the vector plasmid pUC18 were kanamycin- and ampicillin-sensitive. Plasmid DNA showed a clear increase in size from $2.7 \mathrm{~Kb}$ to $3.4 \mathrm{~Kb}$ after insertion of the $d p s$ gene, and PCR amplification of $d p s$ showed a distinct band at 790bp in both the control genomic DNA and the cloned plasmid, which is in agreement with the projected size of the $d p s$ gene and promoter region (Figure 3.2). Positive transformants were sequence compared to the EDL933 dps gene, and showed $91 \%$ similarity, confirming the insertion of $d p s$ into the MCS of pUC18. 


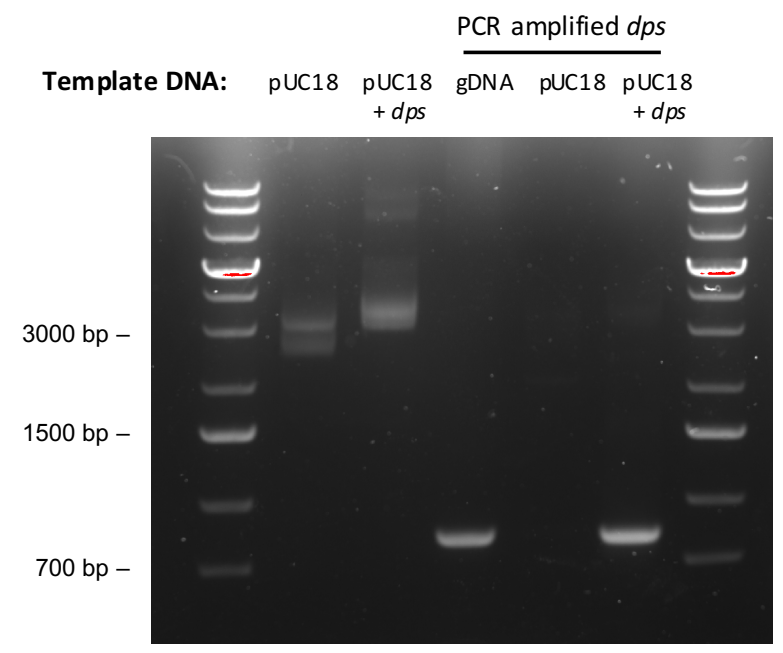

Figure 3.2: Cloning of $\boldsymbol{d p s} \mathrm{ORF}+$ promoter into vector plasmid pUC18. Insertion of the $d p s$ ORF $+\mathrm{P}$ sequence into the pUC18 plasmid, as validated through mini-prep (lanes $2 \& 3$ ) and PCR-amplification of dps (lanes 4-6) on a $0.8 \%$ agarose gel.

\subsubsection{Peptide wrwycr behaves as a bactericidal antibiotic}

Survival assays of EHEC EDL933 WT exposed to various antibiotics showed a 1.5-log fold increase in survival when exposed to the control media (MHB) containing no antibiotics and no change in survival when exposed to spectinomycin, a bacteriostatic antibiotic (Figure 3.3a). In contrast, when EHEC was exposed to either kanamycin, a bactericidal antibiotic, or peptide wrwycr there was a 5-log fold decrease in survival, demonstrating a significant level of bacterial killing. Similarly, flow cytometry analysis showed an increase in hydroxyl radical formation in cells treated with either kanamycin or peptide wrwycr, but not with either MHB or spectinomycintreated cells (Figure 3.3b-c), consistent with previous studies that show an increase in hydroxyl radical formation in the presence of bactericidal but not bacteriostatic antibiotics (Kohanski et al., 2007). These results indicate that peptide wrwycr behaves similarly to kanamycin, and more broadly, to the bactericidal class of antibiotics. 


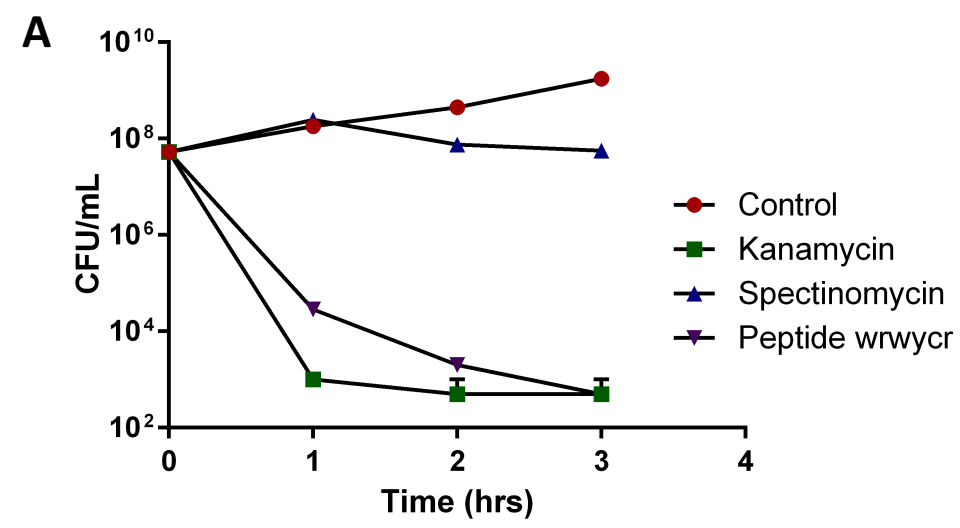

B
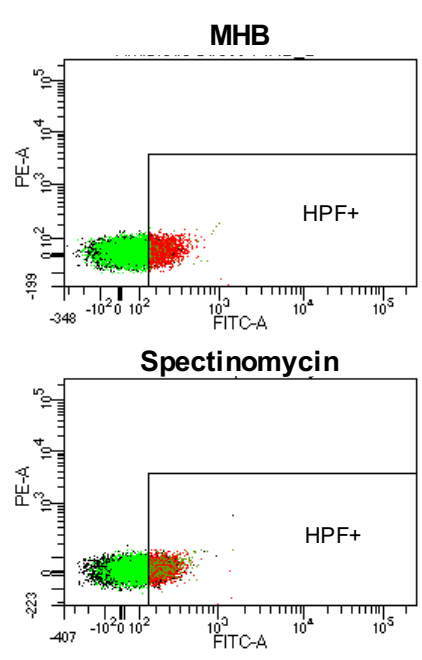
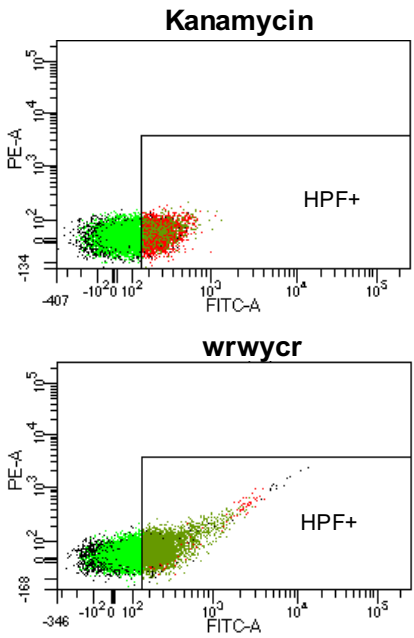

C

\begin{tabular}{|c|c|}
\hline Treatment & HPF Signal \\
\hline MHB & 232 \\
\hline Kanamycin & 270 \\
\hline Spectinomycin & 238 \\
\hline Peptide wrwycr & 339 \\
\hline
\end{tabular}

Figure 3.3: Comparison of peptide wrwyer to bactericidal and bacteriostatic antibiotics. (a) Survival of EHEC exposed to MHB broth containing either no antibiotics, $5 \mu \mathrm{g} / \mathrm{mL}$ kanamycin, $400 \mu \mathrm{g} / \mathrm{mL}$ spectinomycin, or $50 \mu \mathrm{M}$ peptide wrwycr for 3 hours at $37^{\circ} \mathrm{C}$, with shaking. (b) Flow cytometric analysis of hydroxyl radical formation in EHEC exposed to antibiotics using hydroxyphenyl fluorescein (HPF), as outlined in a. (c) Quantified HPF signal from b.

\subsubsection{Disruption of $d p s$ makes EHEC hypersensitive to acid, peptide, and peptide-acid}

\section{treatment}

When EHEC EDL933 WT was exposed to acid stress at pH 3.0, a time-dependent decrease in viability was seen, with only $15 \%$ of the bacteria surviving 60 min of exposure (Figure 3.4ab). Disruption of $d p s$ further enhanced this decrease in survival by 1 -log fold, with only $4 \%$ of 
bacteria surviving $60 \mathrm{~min}$ of exposure, while complementation of the $d p s$ gene restored survival to wild-type levels. The enhancement of bacterial killing seen in the $d p s$ mutant compared to wildtype EHEC was more pronounced at the lower $\mathrm{pH}$ of 2.5, where a 2-log fold difference in survival was seen between the two strains (data not shown).

Similarly, when EHEC was exposed to 50uM peptide wrwycr treatment, a 2-log fold decrease in survival was observed for WT bacteria (Figure 3.4c). Disruption of $d p s$ enhanced this decrease in survival by 2-log fold, for a total 4-log fold decrease in survival compared to $t=0$, and complementation restored survival to wild-type levels. Survival of the $d p s$ mutant containing the empty vector, pUC18, matched that of the $d p s$ mutant strain.

Finally, when EHEC was pre-treated with peptide for $15 \mathrm{~min}$ and then exposed to acute acid stress (pH 3.0), there was a greater decrease in survival over the 60 min acid treatment (Figure 3.4d). Reduction in viability was 3.5-log fold for wild-type EHEC, while disruption of the $d p s$ gene further increased this to a 5.5-log fold decrease in survival compared to $\mathrm{t}=0$. Complementation of $d p s$ restored survival to wild-type levels, while the presence of the empty vector showed survival similar to that of the $d p s$ mutant strain. These results suggest that disruption of $d p s$ makes EHEC hypersensitive to acid, peptide, and peptide-acid treatment, consistent with previous studies indicating a protective role of Dps against cellular stress (Almirón et al., 1992; Altuvia et al., 1994; Jeong et al., 2008; Nair and Finkel, 2004). 

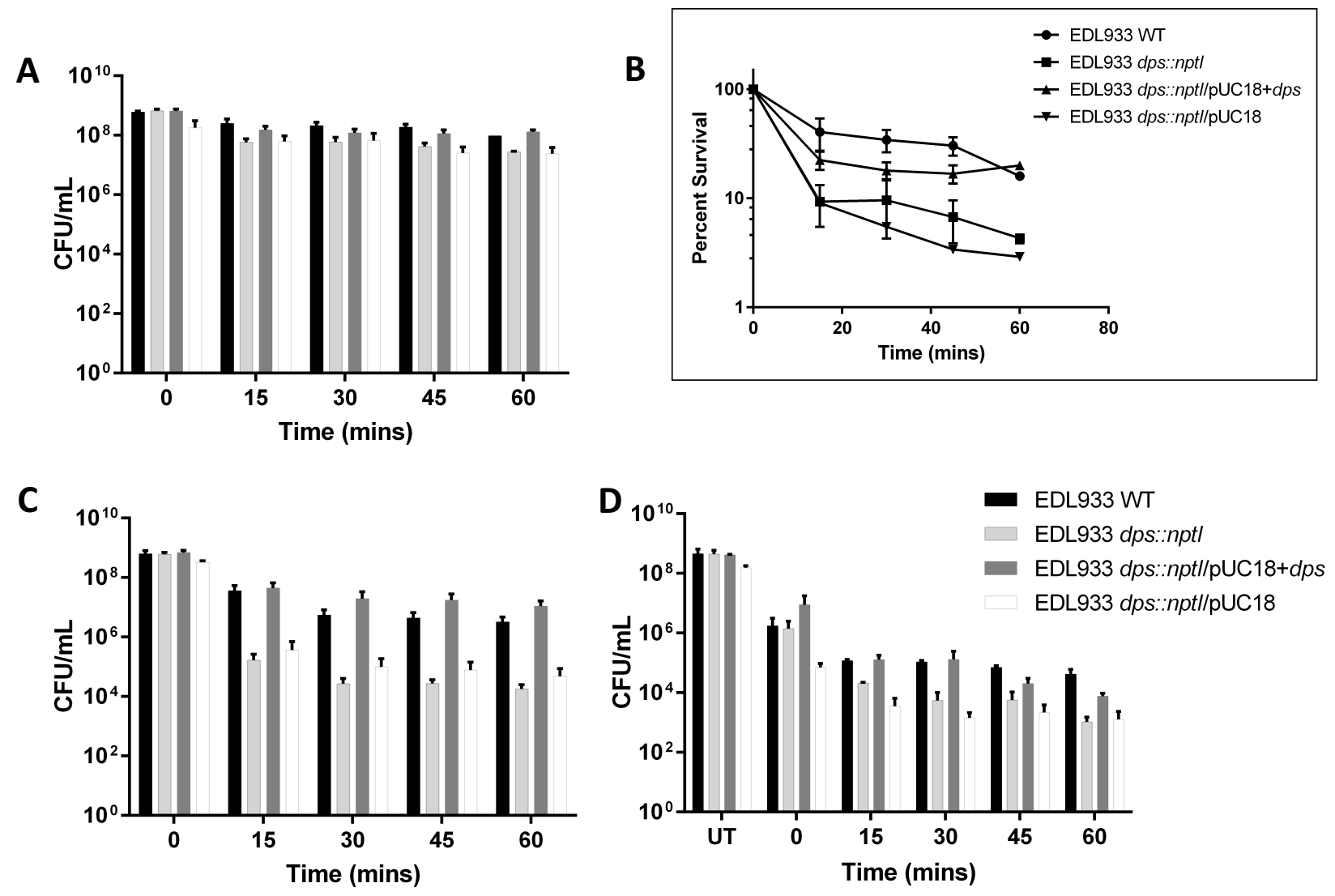

Figure 3.4: Survival of EHEC following acid, peptide, or peptide-acid treatment. Survival assays showed CFU/mL or percent survival after 1 hour (a-b) acid (pH 3.0) stress; or (c) peptide wrwycr (50 $\mu \mathrm{M})$ treatment; or (d) peptide-acid treatment (15 min peptide pre-treatment at $50 \mu \mathrm{M}$ followed by 1 hour acid stress at $\mathrm{pH}$ 3.0). Data bars represent means \pm SEMs, $n=2$.

\subsubsection{Hydroxyl radical formation is increased following peptide treatment of EHEC}

When EHEC EDL933 WT was treated with peptide wrwycr, a distinct increase in hydroxyl radical formation was observed, as seen by flow cytometric analysis (Figure 3.5) with the HPF signal increasing from 256 to 282 . This increase was even more pronounced in the absence of $d p s$, with the HPF signal increasing to 306 , and was restored in the complemented strain. These results suggest that Dps is playing a significant role in protecting EHEC against peptide-induced hydroxyl radical formation. 
A
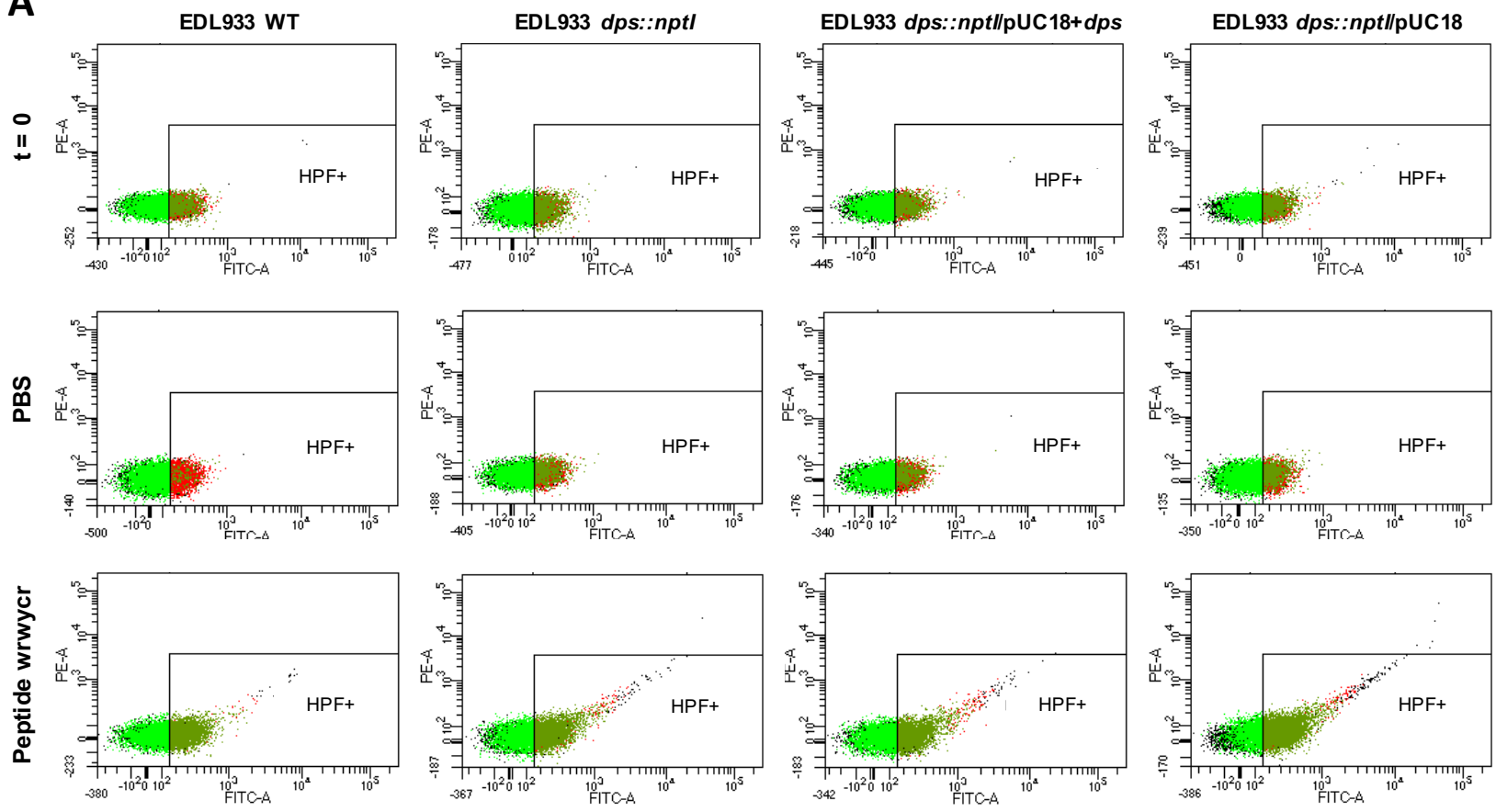

B

\begin{tabular}{|c|c|c|c|c|}
\hline & EDL933 WT & EDL933 dps::nptI & $\begin{array}{c}\text { EDL933 } \\
\text { dps::nptI/pUC18+dps }\end{array}$ & $\begin{array}{c}\text { EDL933 } \\
\text { dps::nptI/pUC18 }\end{array}$ \\
\hline $\mathbf{t}=\mathbf{0}$ & 256 & 258 & 256 & 248 \\
\hline PBS & 257 & 248 & 236 & 234 \\
\hline $\begin{array}{l}\text { Peptide } \\
\text { wrwycr }\end{array}$ & 282 & 306 & 289 & 336 \\
\hline
\end{tabular}

Figure 3.5: Hydroxyl radical formation in peptide-treated EHEC. (a) Flow cytometric analysis of hydroxyl radical formation using hydroxyphenyl fluorescein (HPF) following 50 $\mu \mathrm{M}$ peptide wrwycr treatment for 1 hour at room temperature. (b) Quantified HPF signal from a.

\subsection{DISCUSSION}

This is the first study to date to classify peptide wrwycr's mechanism of bacterial killing as that of the bactericidal class of antibiotics, and to demonstrate a relationship between peptide wrwycr's different antimicrobial roles through the protective mechanisms of the DNA binding 
protein, Dps. The data presented here indicate that treatment of EHEC with peptide wrwycr induces bacterial cell death and increases hydroxyl radical formation to levels similar to that seen with the bactericidal, but not bacteriostatic, class of antibiotics. These results suggest that, like other bactericidal antibiotics, peptide wrwycr may act by breaking down iron-sulfur clusters within the bacterial cell to release $\mathrm{Fe}^{2+}$, thereby driving the Fenton reaction to form hydroxyl radicals and eventually leading to bacterial cell death (Kohanski et al., 2007; Orchard et al., 2012).

The survival assays demonstrated that disruption of the $d p s$ gene made EHEC significantly more sensitive to acute acid stress $(\mathrm{pH} 3.0)$, peptide treatment $(50 \mu \mathrm{M})$, or a combination of peptide-acid treatment during stationary phase of growth. The hypersensitivity of the $d p s$ mutant to acute acid exposure was profoundly enhanced at a lower $\mathrm{pH}$ of 2.5 . Since both of the $\mathrm{pH}$ values tested are acidic enough to induce significant levels of DNA damage, the results are consistent with previous reports that $d p s$ directly binds DNA to protect against acid-induced DNA damage (Choi et al., 2000; Jeong et al., 2008). Similarly, the hypersensitivity of the $d p s$ mutant to peptide treatment can be explained by the bactericidal activity of the peptide, in which $\mathrm{Fe}^{2+}$ is released and leads to increased levels of hydroxyl radicals within the cell. Since $d p s$ expression is known to be induced and sequester $\mathrm{Fe}^{2+}$ in response to hydroxyl radical formation, disruption of $d p s$ would result in excess levels of $\mathrm{Fe}^{2+}$ present within the cell, thereby leading to higher levels of hydroxyl radicals and an increase in bacterial cell death (Kohanski et al., 2007). The combination of peptideacid treatment combines both of these effects, and therefore requires two mechanisms of DNA protection from Dps - DNA binding and ferroxidase activity (Chiancone and Ceci, 2010). This is supported by the enhanced level of bacterial killing seen in both the wild-type and $d p s$ mutant strains when exposed to peptide-acid treatment. 
In summary, the findings of this study extend our previous findings of peptide wrwycr's potential as an antimicrobial agent by demonstrating its action to be similar to that of the bactericidal class of antibiotics, and provide a unique link between the two major antimicrobial roles of this peptide through the DNA-binding protein Dps.

\subsection{CONCLUSIONS \& FUTURE DIRECTIONS}

\subsubsection{Conclusions}

We have successfully demonstrated that the DNA-binding protein, Dps, plays a vital protective role against peptide wrwycr treatment of EHEC through its DNA-binding and ferroxidase center activity. Disruption of the $d p s$ gene resulted in hypersensitivity to each of acute acid exposure, peptide treatment, and peptide-acid treatment compared to the wild-type strain. Moreover, flow cytometric analysis demonstrated increased levels of hydroxyl radical formation in peptide-treated EHEC compared to untreated EHEC, and this was further enhanced in the absence of $d p s$. It is of importance to note that the flow cytometric data may be an underrepresentation of hydroxyl radical formation in each of the treatment groups due to the short lifetime of hydroxyl radicals in the cell. Taken together, these results suggest that upon acid or peptide exposure of EHEC, Dps is upregulated and acts by directly binding DNA to protect against acid-induced DNA damage and by sequestering $\mathrm{Fe}^{2+}$ to prevent the formation of hydroxyl radicals through the Fenton reaction. Additionally, we have also demonstrated that the peptide wrwycr acts similarly to the bactericidal class of antibiotics by causing significant levels of bacterial killing and inducing hydroxyl radical formation. 


\subsubsection{Future Directions \& Significance}

This study sheds light on one of the protective mechanisms employed by EHEC upon exposure to peptide treatment through Dps. Since the findings of this study show that Dps acts to protect EHEC against peptide treatment, and disruption of the $d p s$ gene results in significantly enhanced levels of bacterial killing, this may suggest a unique strategy to enhance the efficacy of peptide treatment. For example, the combination of peptide treatment with another drug or antibody treatment that neutralizes and/or counteracts the protective effects of Dps may enhance peptide-induced bacterial killing, thereby enhancing its ability as a prevention strategy against EHEC infection. Additionally, while this study demonstrates a unique link between the different antimicrobial effects of peptide wrwycr through the protective action of Dps, there are likely other protective mechanisms at play. A deeper analysis of these protective mechanisms and antimicrobial resistance mechanisms is vital to fully understanding how EHEC responds to peptide treatment. While our previous studies did not demonstrate acquired resistance to peptide treatment, analysis of antimicrobial resistance mechanisms such as expression of efflux pumps, changes in cell membrane permeability, and alterations of antimicrobial target proteins would allow us to gain better insight into how EHEC changes its metabolism in response to this antimicrobial. This insight would allow for the development of combination drug treatments that target the antimicrobial resistance mechanisms at play to enhance the efficacy of peptide treatment. 
In conclusion, this research sheds light on how Dps provides a unique link between the different antimicrobial roles of peptide wrwycr treatment, by binding DNA directly to protect against acid-induced DNA damage and by its ferroxidase activity to sequester $\mathrm{Fe}^{2+}$ and protect against iron toxicity and hydroxyl radical-mediated cell death. Moreover, these results provide an interesting opportunity to develop a combination drug therapy that targets Dps as a means to enhance peptide-induced bacterial killing. 


\title{
Chapter 4: Modulation of EHEC 0157:H7 Virulence by
}

\author{
Short Chain Fatty Acids
}

Lackraj, T.*, Kim, J.*, Tran, S., \& Barnett Foster, D. (2016) Differential modulation of flagella expression in enterohemorrhagic Escherichia coli O157:H7 by intestinal short chain fatty acid mixes. Microbiology doi:10.1099/mmic.0.000357.

* Co-first authors; Editor's Choice 


\subsection{ABSTRACT}

During passage through the human gastrointestinal tract, enterohemorrhagic Escherichia coli (EHEC) encounters various microenvironmental stresses, including acute acid stress in the stomach, bile salt stress in the small intestine, and short chain fatty acid (SCFA) stress in the small and large intestines. Each of these microenvironmental stresses produces unique antimicrobial conditions that EHEC must overcome prior to reaching its desired site of colonization in the distal colon. While EHEC uses various strategies to combat and survive these host assaults, it may also use them as chemical cues about the local microenvironment in order to modulate its virulence accordingly. Of particular interest to this study is how EHEC may utilize changing concentrations of SCFAs in the small and large intestines to modulate virulence as it approaches the site of colonization, and specifically how it modulates flagella expression and motility in response to SCFAs. In this study, we investigate the impact of exposure to short chain fatty acid mixtures, which simulate the concentrations and compositions within the small and large intestine, on EHEC flagella expression and function. Using a combination of transcriptome analysis, quantitative realtime PCR, immunofluorescence, and immunoblot analyses, we show that when EHEC is exposed to low concentrations $(30 \mathrm{mM})$ of SCFAs representative of the small intestine, there is a strong significant upregulation of flagellar genes and the key flagellar protein FliC. By contrast, when EHEC is exposed to high concentrations $(172 \mathrm{mM})$ of SCFAs representative of the large intestine, there is a significant downregulation of flagellar genes and FliC protein expression. Similarly, EHEC motility is also modulated in response to these different SCFA mixes. Interestingly, the impact of the individual SCFAs within each mix is not equivalent to the response of the SCFA mixes, suggesting that the specific composition of each mix is vital to the regulatory response. Collectively, these results demonstrate that EHEC modulates flagella expression and motility 
differentially in response to varying concentrations of SCFAs typical of the small and large intestines.

\subsection{INTRODUCTION}

\subsubsection{Short Chain Fatty Acids and Colonic Health}

The human gastrointestinal tract is comprised of multiple distinct microenvironmental niches. In particular, the environment of the small and large intestines are characterized by changing concentrations of short chain fatty acids (SCFAs). SCFAs are a major group of metabolic end products from the fermentation of dietary fibers, protein, and peptides (Cummings, 1983; Roy et al., 2006; Smith and Bryant, 1979; Tobe et al., 2011). Since humans lack the enzymes necessary for degradation of the majority of dietary fibers, these non-digestible carbohydrates pass through the upper GI tract to the cecum and large intestine where they are fermented by the anaerobic cecal and colonic microbiota (den Besten et al., 2013; Roy et al., 2006). By definition, SCFAs are saturated aliphatic organic acids consisting of one to six carbons, and are most abundantly ( $>95 \%)$ made up of acetate, butyrate, and propionate (Figure 4.1) (Cook and Sellin, 1998; Cummings et al., 1987; Cummings and Macfarlane, 1991; den Besten et al., 2013; Macfarlane et al., 1992; Roy et al., 2006; Tobe et al., 2011).
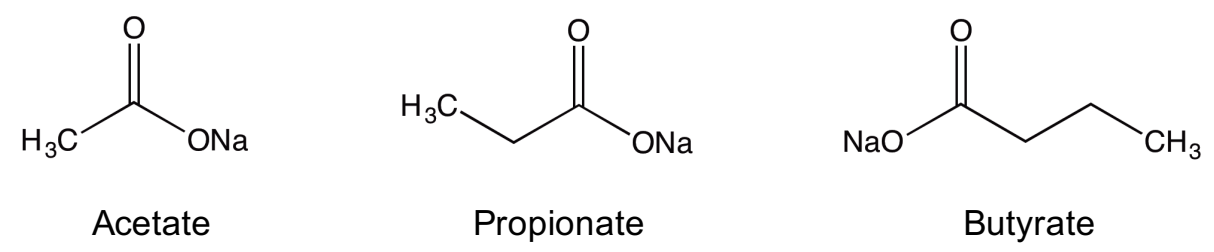

Figure 4.1: Chemical structure of SCFAs. The three most abundant SCFAs present through the breakdown of dietary fiber are acetate $(\mathrm{C} 2)$, propionate $(\mathrm{C} 3)$, and butyrate $(\mathrm{C} 4)$. 
Although the relative concentration and composition of SCFA mixes in the lumen of specific regions along the human GI tract are broad and dynamic over time, the overall trend shows that SCFA levels are lowest in the small intestine, ranging from approximately 20 to $40 \mathrm{mM}$, and increasing dramatically in the cecum and colon to concentrations ranging between 160 to $200 \mathrm{mM}$ (Herold et al., 2009; Lawhon et al., 2003; Macfarlane et al., 1992). Within the large intestine, total SCFA concentrations range from 70 to $140 \mathrm{mM}$ in the proximal colon and 20 to $70 \mathrm{mM}$ in the distal colon, with acetate being the most abundant (Cummings and Macfarlane, 1991; den Besten et al., 2013; Tobe et al., 2011; Topping and Clifton, 2001). The total concentrations and composition of SCFAs within the GI tract are affected mainly by two vital aspects: diet (the amount and type of food consumed) as well as the composition of the gut microbiota (den Besten et al., 2013; Flint et al., 2008; Herold et al., 2009).

The human diet, specifically the amount and type of dietary fiber consumed, constitutes one of the major determinants of SCFA concentration and composition present within the intestinal lumen (Fernandes et al., 2014; Flint et al., 2008). The average human diet in western societies contains approximately 20-25 grams of fiber per day, and may reach as high as 60 grams per day in diets rich in fruits and vegetables (Bingham et al., 2003; den Besten et al., 2013; Musso et al., 2011). The specific type of fiber consumed affects the relative SCFA composition. For example, the fermentation of starch results in high levels of butyrate, whereas the fermentation of pectin results in higher levels of acetate (Cummings and Macfarlane, 1991; Gilbert et al., 2005; Herold et al., 2009).

Additionally, the composition of the gut microbiota constitutes the second major determinant of SCFA concentration and composition. The three most abundant phyla present in the intestine, Bacteriodetes, Firmicutes, and Actinobacteria, have differential impacts on SCFA 
composition. Specifically, the Bacteriodetes phylum mainly produce acetate and propionate as its metabolic end product, whereas the Firmicutes phylum primarily produces butyrate (den Besten et al., 2013; Macfarlane and Macfarlane, 2003). The microbial species present within the lower GI tract produce SCFAs as a necessary waste product required to balance redox equivalent production in the anaerobic environment of the gut (van Hoek and Merks, 2012).

Interestingly, the amount and type of fiber intake plays a vital role in the composition of the gut microflora, and consequently the SCFA on the type and amounts of SCFAs produced (den Besten et al., 2013; Fernandes et al., 2014). Dietary fibers which result in high concentrations of SCFAs cause a decrease in colonic $\mathrm{pH}$, which subsequently affects the composition of the colonic microbiota and further SCFA production. The decrease in luminal $\mathrm{pH}$ is a result of the exchange of SCFAs by the host cells for bicarbonate (den Besten et al., 2013). The pH-induced changes in the microbiota within the colon are a vital aspect of SCFA production, especially within the proximal colon where the highest amount of bacterial activity occurs. Similarly, this is also where the greatest amount of carbohydrate substrates is available, making the proximal part of the colon the principle site of fermentation (den Besten et al., 2013; Topping and Clifton, 2001).

In addition to the impact of diet and gut microflora on SCFA concentration, the time after digestion also plays a vital role. While the cecum yields approximately 400 to $600 \mathrm{mmol}$ of SCFAs each day through the fermentation of carbohydrates, the total concentration decreases rapidly as almost $95 \%$ of SCFAs are absorbed by the colonocytes for use as 5-10\% of the total dietary energy (Bergman, 1990; Dawson et al., 1964; Erickson and Doyle, 2007; Roediger, 1982; Royall et al., 1990; Ruppin et al., 1980; Topping and Clifton, 2001).

In addition to its multitude of applications including artificial flavourings, pharmaceutical agents, and plasticizers for medical and commodity plastics, SCFAs are also widely used as 
antimicrobial agents in the food, cosmetics, and pharmaceutical industries. Moreover, studies have demonstrated that exposure of E. coli to SCFAs slows growth, a finding that suggests a role for SCFAs in enzyme inhibition, cytoplasmic acidification, redox imbalances, and cellular membrane damage (Black and DiRusso, 1994; DiRusso et al., 1999; Jarboe et al., 2013; Lennen et al., 2011; Rodríguez-Moyá and Gonzalez, 2015).

Recently, dietary fibers and their fermentation end product, SCFAs, have been of interest due to their beneficial effects on metabolic functions such as body weight, glucose homeostasis, food intake, and insulin sensitivity (Delzenne and Cani, 2005; Marlett et al., 2002; Nicholson et al., 2012; Venn and Mann, 2004). Interestingly, SCFAs have been implicated to play a role in maintaining colonic health and reducing the risk of irritable bowel syndrome, inflammatory bowel disease, cardiovascular disease, diabetes, and colon cancer (den Besten et al., 2013; Galisteo et al., 2008; Kasubuchi et al., 2015; Tobe et al., 2011; Wong et al., 2006). Moreover, it has been hypothesized that SCFAs may play a key role in the prevention and treatment of these bowel disorders and cancers since butyrate is a major energy source for colonocytes and promotes cell differentiation, and has been shown to protect epithelial cell lines from Campylobacter invasion and translocation (Donohoe et al., 2011; Fukuda et al., 2011; Harig et al., 1989; Van Deun et al., 2008; Wong et al., 2006). Clinical studies have also demonstrated the beneficial effects of SCFA administration in the treatment of ulcerative colitis, Crohn's disease, and antibiotic-associated diarrhea (Binder, 2010). Together, these studies demonstrate a vital role for SCFAs in maintaining colonic health and may suggest a key role for SCFAs in protecting the host against incoming pathogens. 


\subsubsection{Short Chain Fatty Acids and Virulence Gene Expression}

A critical feature of enteric infection involves the ability of the incoming pathogen to tolerate, possibly adapt to, and potentially exploit the various conditions encountered in the local environment through alterations in their physiology and virulence properties. In order to achieve this, pathogens have evolved unique systems to sense these environmental conditions (such as temperature, oxygen, and nutrients) and subsequently transduce the signal in order to reprogram their virulence gene expression accordingly to ensure successful infection (Heroven et al., 2012).

SCFAs have been shown to affect the expression of virulence genes in Salmonella and EHEC (Lawhon et al., 2003; Nakanishi et al., 2009; Tobe et al., 2011). Specifically, the exposure of Salmonella enterica to acetate provides a signal for invasion gene expression through the production of acetyl phosphate in the cytoplasm (Lawhon et al., 2003; Tobe et al., 2011). Additionally, the exposure of EHEC to butyrate, but not to acetate or propionate, enhances virulence gene expression, specifically genes involved in the adherence of EHEC to epithelial cells thereby increasing the adherence capacity of EHEC through increased LEE gene expression. This is achieved through the sensing regulator leucine-responsive regulatory protein (Lrp) (Nakanishi et al., 2009; Tobe et al., 2011). Exposure to butyrate activates the expression of leuO in EHEC through the virulence regulators Pch, Ler, and Lrp. Conversely, LeuO positively regulates transcription of the LEE1 operon, which includes the Ler gene, thereby enhancing virulence gene expression through a positive feedback loop (Takao et al., 2014).

Of interest is the BarA/UvrY two-component system (TCS) in E. coli, which is often utilized by pathogens to sense external signals in the local environment and adapt their bacterial fitness and virulence accordingly. Amongst the various environmental conditions that are sensed by BarA/UvrY are weak acids, specifically formate and acetate, which leads to modulation of the 
carbon storage regulator (Csr) system (Suzuki et al., 2002; Weilbacher et al., 2003). The Csr system is a post-transcriptional regulatory system present in most animal pathogens and has been implicated to have a key role in the adaptation of these pathogens to changing environmental conditions. This system is comprised of a dimeric RNA-binding protein, CsrA, and two independent small non-coding RNAs, CsrB and CsrC, which act as antagonists of CsrA. CsrA is a post transcriptional RNA-binding regulator which functions by binding mRNAs and affecting their translation and/or stability (Babitzke and Romeo, 2007; Wang et al., 2005; Wei et al., 2001).

Typically, the binding of mRNAs by CsrA leads to negative regulation of translation, although it has been shown to have a positive stabilizing effect on the flhDC mRNA in E. coli. FlhDC encodes the master regulator for synthesis of the flagellum, one of the most complex cellular machinery in bacteria (Heroven et al., 2012; Wei et al., 2001). Flagella synthesis involves 14 different operons and over 50 genes. The expression of these genes is tightly regulated in order to control this process, and regulation has been organized into a three-tier hierarchy. FlhDC is at the top of this hierarchy, in class I, and recognizes class II flagellar promoters (Liu and Matsumura, 1994). Genes in class II encode proteins for the basal body and the hook of the flagellum, as well as for FliA, which is required for transcription of class III genes. The genes in this class are required for assembly of the flagellar filament, motor activity, and chemotaxis (Hughes et al., 1993; Wei et al., 2001). Once fully assembled, the flagellum is an intricate multiprotein assembly (Figure 4.2) which is most often characterized by its role in the propulsion or motility of bacteria (Rossez et al., 2015). It follows then that the recognition of acetate through the BarA/UvrY TCS leads to increased expression of $\mathrm{CsrB}$ and $\mathrm{CsrC}$, which leads to inhibition of $\mathrm{Csr} A$ and a subsequent decrease in motility, since the $f l h D C$ mRNA is no longer stabilized through CsrA binding (Suzuki et al., 2002; Weilbacher et al., 2003). This suggests that exposure to acetate, at concentrations high 
enough to be detected by the BarA/UvrY TCS, leads to decreased flagella expression and motility. In contrast, it has also been shown that exposure of EHEC to low concentrations of butyrate simultaneously enhances flagella production, motility, and LEE gene expression. This response of flagella to SCFAs is mediated through two steps of the flagella regulatory hierarchy - through Lrp and independently of FlhDC activation (Takao et al., 2014; Tobe et al., 2011). Taken together, these findings suggest that each individual SCFA may modulate bacterial virulence differentially.

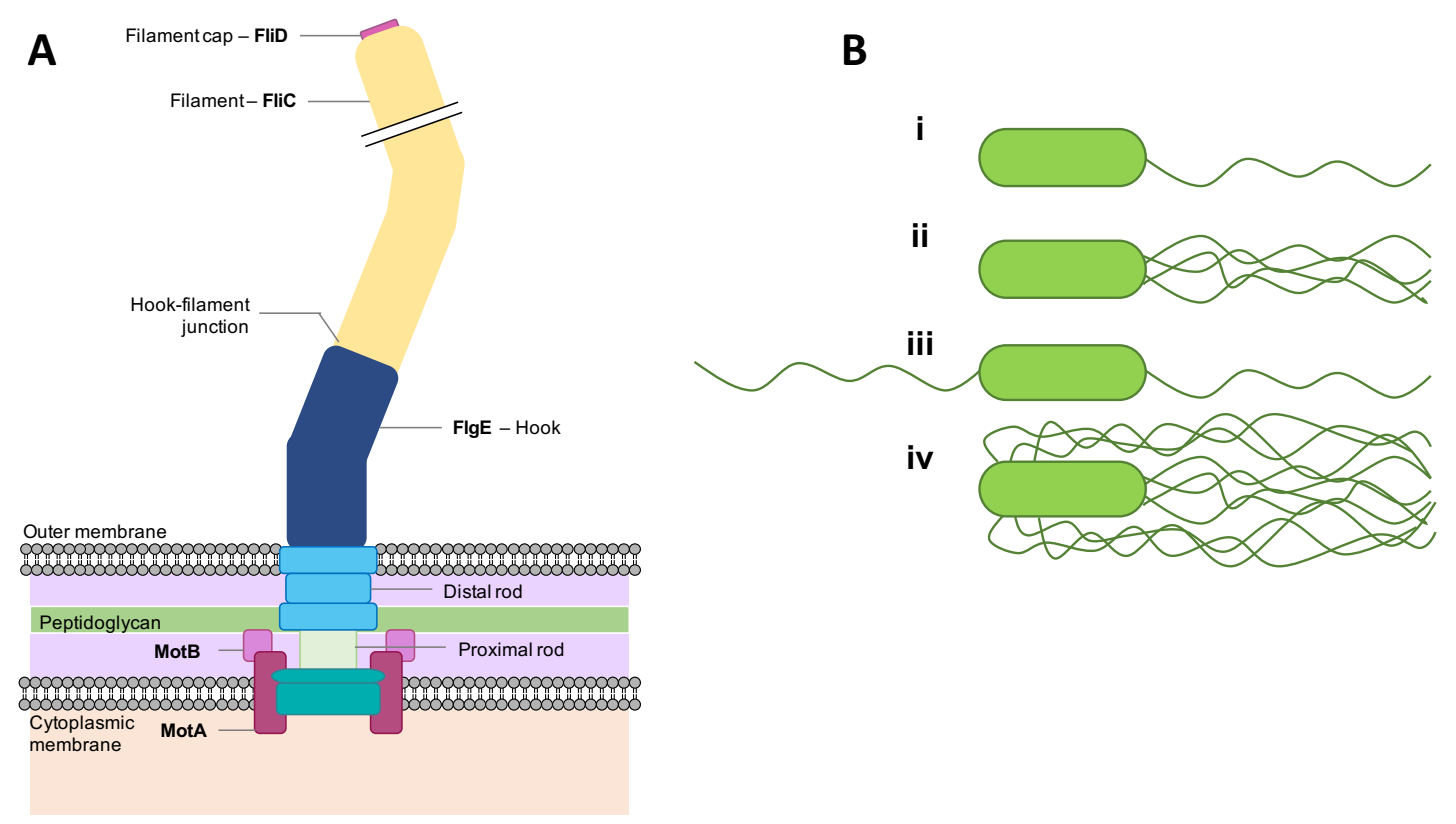

Figure 4.2: Flagella structure and organization. (a) The flagellum is a multiprotein assembled appendange spanning from the bacterial cytoplasm, across the double membrane into the extracellular space. (b) The organization of flagella around the bacterial body can be either i) monotrichous, ii) lophotrichous, iii) amphitrichous, or iv) peritrichous. Adapted from (Chevance and Hughes, 2008).

\subsubsection{Rationale and Overall Aim}

The production of SCFAs, through fermentation of dietary fiber by the gut microflora, results in a unique and dynamic environment within the lower GI tract. The presence of these metabolites may serve as an environmental cue for EHEC to regulate its virulence through several 
complex systems. During transit through the lower GI tract, a vital aspect of EHEC's virulence is motility, which may provide an important advantage in avoiding detrimental locations and successfully reaching its favourable site of colonization in the colon. It follows then that the expression of flagella is important for successful infection, as it is the main driving force behind bacterial motility, but may also serve as adhesive appendages during the initial phases of colonization. The goal of this study is to characterize the effect of differential concentrations of SCFAs, representative of transit through the small and large intestines, on expression of flagella in EHEC and subsequently on motility. Furthermore, since previous studies have demonstrated differential effects of the three major SCFAs, acetate, butyrate, and propionate, this study will examine the effects of each individual SCFA on flagella expression.

\subsection{MATERIALS AND METHODS}

\subsubsection{Bacterial strains and culture conditions}

Escherichia coli O157:H7 strain 86-24 was used in this study. Bacterial glycerol stocks were maintained at $-80^{\circ} \mathrm{C}$, and were streaked onto Luria-Bertani (LB) (BioShop, Burlington, ON, Canada) agar prior to use to obtain single colonies. Overnight cultures were prepared by inoculating single colonies into LB broth, and incubating $12-16 \mathrm{hr}$ at $37^{\circ} \mathrm{C}$ with shaking.

\subsubsection{SCFA treatment of EHEC}

Overnight cultures were diluted in fresh LB containing $100 \mathrm{mM}$ MOPS (morpholinepropanesulfonic acid; $\mathrm{pH}$ 6.7) and either $30 \mathrm{mM} \mathrm{NaCl}$, 30mM SCFA mix (25mM sodium acetate, $2.5 \mathrm{mM}$ sodium butyrate, $2.5 \mathrm{mM}$ sodium propionate) (Herold et al., 2009), or 
$172 \mathrm{mM} \mathrm{NaCl}$ to an $\mathrm{OD}_{600}$ of $0.05,0.05$ or 0.15 respectively. Cultures were then incubated $6 \mathrm{hr}$ at $37^{\circ} \mathrm{C}+5 \% \mathrm{CO}_{2}$ to late exponential phase $\left(\mathrm{OD}_{600}=0.8-1.0\right)$. Due to the prolonged lag phase of EHEC incubated with the 172mM SCFA mix, the overnight culture was diluted in fresh LB containing $100 \mathrm{mM}$ MOPS to an $\mathrm{OD}_{600}$ of 0.2 , then incubated at $37^{\circ} \mathrm{C}+5 \% \mathrm{CO}_{2}$ for $2 \mathrm{hr}$ prior to addition of the $172 \mathrm{mM}$ SCFA mix ( $95 \mathrm{mM}$ sodium acetate, $17 \mathrm{mM}$ sodium butyrate, $60 \mathrm{mM}$ sodium propionate) (Herold et al., 2009) and continued growth to late exponential phase.

For treatment of EHEC with individual SCFAs, solutions of sodium acetate, sodium butyrate, and sodium propionate were either prepared to a set concentration of $30 \mathrm{mM}$ or $172 \mathrm{mM}$ or set to the original concentrations within the original $30 \mathrm{mM}$ or $172 \mathrm{mM}$ mixes. Bacterial cultures were diluted in fresh LB containing 100mM MOPS and the appropriate stress and then incubated as described above. All cultures with a concentration of SCFA larger than 30mM was set to an $\mathrm{OD}_{600}$ of 0.2 and incubated for $2 \mathrm{hr}$ prior to addition of the SCFA stress.

A concentration gradient of individual SCFAs $(0,30,60,90,130$, and $170 \mathrm{mM})$ was prepared and bacterial cultures were diluted in fresh LB containing 100mM MOPS and the appropriate stress and incubated as described above. All cultures with a concentration of SCFA larger than $30 \mathrm{mM}$ was set to an $\mathrm{OD}_{600}$ of 0.2 and incubated for $2 \mathrm{hr}$ prior to addition of the SCFA stress.

\subsubsection{Quantitative real-time PCR analysis of gene expression}

Following SCFA treatment, total RNA was isolated using the GeneJet RNA Purification

Kit (ThermoFisher Scientific, Waltham, MA, USA) and converted to cDNA using the iScript cDNA synthesis kit (BioRad, Mississauga, ON, Canada) according to manufacturers' instructions. Gene expression was assessed using primers against gapA (control), fliC, flhC, flhD, motAB, cheA, and $c h e W$ (see Table 4.1 for primer sequences), Roche LightCycler FastStart DNA Master SYBR 
Green I, and Roche LightCycler 2.0 Instrument (Roche, New York, NY, USA). At least 3 independent experiments were carried out for each primer set.

Table 4.1: Primer sequences used for quantitative real-time PCR.

\begin{tabular}{|c|c|c|}
\hline Primer & Sequence (5' $\rightarrow$ 3') & Reference \\
\hline gapA_F & AGGTCTGATGACCACCGTTC & \multirow{2}{*}{ This study } \\
\hline gapA_R & GGAACGCCATACCAGTCAGT & \\
\hline fliC_F & CAAGTTGCCTGCATCGTCTA & \multirow{2}{*}{ This study } \\
\hline fliC_R & TCAGCTTCAAAACGTGATGC & \\
\hline flhC_F & GTGGGATAATATCGGCAGGA & \multirow{2}{*}{ This study } \\
\hline flhC_R & GCGGTTTGTTGAAAGTGGAT & \\
\hline flhD_F & GATGCCGGTATGAATTTGCT & \multirow{2}{*}{ This study } \\
\hline flhD_R & CAGCGTCTGATTGTTCAGGA & \\
\hline motAB_F & TTCTAAACATCGGGCGATTC & \multirow{2}{*}{ This study } \\
\hline motAB_R & CAGGGGGAAGTGAATAAGCA & \\
\hline cheA_F & CTGATGTTCGGCAGACAGAA & \multirow{2}{*}{ This study } \\
\hline cheA_R & AACTCGGCAAGCAGGTAGAA & \\
\hline cheW_F & CGTCAATGAAAGCACGTCTG & \multirow{2}{*}{ This study } \\
\hline cheW_R & AGGTAACACGGATTGCGAAC & \\
\hline
\end{tabular}

\subsubsection{Immunoblot analysis of FliC expression}

Following SCFA treatment, bacteria were collected by centrifugation at 3,500rpm for $10 \mathrm{~min}$ at $4{ }^{\circ} \mathrm{C}$, washed twice in $1 \mathrm{X}$ PBS $\left(137 \mathrm{mM} \mathrm{NaCl}, 2.7 \mathrm{mM} \mathrm{KCl}, 10 \mathrm{mM} \mathrm{Na}_{2} \mathrm{HPO}_{4}, 1.8 \mathrm{mM} \mathrm{KH}_{2} \mathrm{PO}_{4}\right)$, and re-suspended in $1 \mathrm{X}$ sodium dodecyl sulfate (SDS) sample buffer $(10 \%$ Glycerol, $60 \mathrm{mM}$ Tris/ $\mathrm{HCl} \mathrm{pH} 6.8,2 \%$ SDS, $0.01 \%$ bromophenol blue, $1.25 \% \beta$-mercaptoethanol). Samples were run on a $10 \%$ SDS-polyacrylamide gel ( $70 \mathrm{~V}$ for $30 \mathrm{mins}, 120 \mathrm{~V}$ for $90 \mathrm{mins}$ ) and transferred onto a 45 $\mu \mathrm{m}$ Immobilon-P PVDF membrane (EMD Millipore, Etobicoke, ON, Canada) (100V for $2 \mathrm{hr}$ at $4{ }^{\circ} \mathrm{C}$ ). Membranes were blocked in 5\% TBST + milk for $1 \mathrm{hr}$ at room temperature with shaking, and incubated overnight at $4^{\circ} \mathrm{C}$ in either anti-H7 (1:1000 dilution; Denka-Seiken, Tokyo, Japan) 
or anti-DnaK (1:10,000 dilution; Enzo Life Sciences, Farmingdale, NY, USA). This was followed by incubation with a horseradish peroxidase-conjugated secondary antibody (1:10,000 dilution; Bethyl Laboratories Inc., Montgomery, TX, USA) and visualization using ECL detection. At least 3 independent experiments were carried out for each condition.

\subsubsection{Soft-agar motility assays}

The effect of SCFA treatment on EHEC motility was assessed by soft agar motility assay using $0.25 \%$ Bacto $^{\mathrm{TM}}$ Tryptone agar plates supplemented with the appropriate SCFA mixtures or $\mathrm{NaCl}$ controls. After SCFA treatment, $2 \mu \mathrm{L}$ of EHEC was spotted onto the soft-agar plates and incubated at $37^{\circ} \mathrm{C}$ for 12 hours, followed by measurement of the motility halo diameter. At least 3 independent experiments were carried out for each condition.

\subsubsection{Statistical analysis}

All data are represented as means \pm standard error of the means (SEMs) of at least 3 independent experiments. Either one-way or two-way analysis of variance (ANOVA) with Tukey's multiple-comparison test was used to determine differences among multiple groups. A $P$ value of $<0.05$ was considered significant.

\subsection{RESULTS}

\subsubsection{Transcriptome analysis of SCFA-treated EHEC}

In order to determine the impact of differential short chain fatty-acid treatment on EHEC O157:H7 (strain EDL933) gene expression, a DNA microarray was carried out using either 30mM 
or 172mM SCFA treatment; the full dataset is available through the GEO database (GEO accession no. GSE79509). For each treatment, the expression of each gene was normalized to its expression in the matching sodium chloride control samples. With a cut-off value of 2 for the fold change in expression $(P<0.05)$, we found that 104 genes were upregulated and 106 genes were downregulated 2-fold or more after exposure to the lower concentration of SCFA relative to its $\mathrm{NaCl}$ control (data not shown). In contrast, 143 genes were upregulated and 214 downregulated following exposure to the higher concentration of SCFA relative to its $\mathrm{NaCl}$ control. Interestingly, the vast majority of flagellar genes showed differential expression in response to the two SCFA treatments, with flagellar genes being upregulated 1.5-4.3 fold by 30mM SCFA and downregulated 3-23 fold by 172mM SCFA (Table 4.2).

Table 4.2: Flagellar genes as determined by DNA microarray ${ }^{\ddagger}$.

\begin{tabular}{lllll}
\hline Gene & Gene Description & Class & $\mathbf{3 0 m M}$ & $\mathbf{1 7 2} \mathbf{m}$ \\
\hline$f \lg A$ & Flagellar basal body P-ring biosynthesis protein & 2 & 2.33 & -6.60 \\
$f l g B$ & Flagellar basal body rod protein & 2 & $*$ & -23.53 \\
$f l g C$ & Flagellar basal body rod protein & 2 & 2.00 & -20.37 \\
$f l g D$ & Flagellar hook assembly protein & 2 & 2.08 & -15.68 \\
$f l g E$ & Flagellar hook protein & 2 & 2.37 & -14.55 \\
$f l g G$ & Flagellar basal body rod protein & 2 & 2.26 & -20.50 \\
$f l g H$ & Basal body L-ring lipoprotein & 2 & 2.28 & -11.98 \\
$f l g I$ & Flagellar basal body P-ring protein & 2 & 1.78 & -10.22 \\
$f l g J$ & Flagellar rod assembly protein & 2 & 2.11 & -7.09 \\
$f l g K$ & Flagellar hook filament junction protein & 2 & 2.50 & -8.43 \\
$f l g M$ & Anti-FliA: FlhD regulator & 2 & 2.23 & $*$ \\
$f l g N$ & Intiator of flagellar filament assembly & 2 & 2.24 & $*$ \\
$f l h C$ & Transcriptional activator & 1 & 2.50 & $*$ \\
$f l h D$ & Transcriptional activator & 1 & 2.40 & $*$ \\
$f l h E$ & Flagella protein & 2 & 2.98 & -12.24 \\
$f l i A$ & Alternative sigma factor 28 & 2 & 2.62 & -21.00 \\
$f l i C$ & Flagellin & 3 & 1.75 & $*$ \\
$f l i F$ & Flagellar basal body MS-ring \& collar protein & 2 & 1.94 & -11.37
\end{tabular}




$\begin{array}{lllll}\text { fliG } & \text { Flagellar motor switch protein } & 2 & 2.02 & -11.78 \\ \text { fliI } & \text { Flagellum specific ATP synthase } & 2 & 2.52 & -9.12 \\ \text { fli } & \text { Flagellar rod assembly protein } & 2 & 2.28 & -17.14 \\ \text { fliK } & \text { Flagellar hook length control protein } & 2 & 2.37 & -9.66 \\ \text { fliL } & \text { Flagellar basal body -associated protein } & 2 & 2.12 & -10.50 \\ f l i M & \text { Flagellar motor switching } & 2 & 2.27 & -13.02 \\ f l i N & \text { Flagellar switch protein } & 2 & 2.22 & -10.29 \\ \text { fliO } & \text { Flagellar biosynthesis protein } & 2 & 2.82 & -10.05 \\ \text { fliP } & \text { Flagellar biosynthesis protein } & 2 & 2.71 & -16.38 \\ \text { fliQ } & \text { Flagellar biosynthesis protein } & 2 & 2.81 & -4.43 \\ \text { fliR } & \text { Flagellar biosynthesis: export pore protein } & 2 & 4.27 & -9.88 \\ \text { fliZ } & \text { RpoS antagonist } & 2 & 1.90 & -10.92 \\ m o t A & \text { Proton conductor component of flagella motor } & 3 & 1.58 & * \\ m o t B & \text { Protein that enables flagellar motor rotation } & 3 & 1.76 & * \\ \text { che } A & \text { Chemotaxis response regulator } & 3 & 1.26 & * \\ \text { cheW } & \text { Purine-binding chemotaxis protein } & 3 & 1.44 & *\end{array}$

Significant fold changes for flagellar genes under $30 \mathrm{mM}$ and $172 \mathrm{mM}$ SCFA treatments relative to their respective sodium chloride controls $(P<0.05)$. * indicates a non-significant change. ${ }^{\ddagger}$ The DNA microarray data presented was conducted by Dr. Seav-Ly Tran (University of Cambridge, Cambridge, UK).

Quantitative real-time PCR confirmed the changes in gene expression of selected flagellar genes following exposure to either $30 \mathrm{mM}$ or $172 \mathrm{mM}$ SCFA treatment. Specifically, fliC, the primary structural flagella gene, showed a consistent 1.64 fold upregulation following $30 \mathrm{mM}$ SCFA treatment relative to $172 \mathrm{mM}$ SCFA treatment. Similarly, $f l h C$ and $f l h D$, the master regulatory genes were significantly upregulated 2.95 and 2.37 fold respectively by 30mM SCFA treatment relative to $172 \mathrm{mM}$ SCFA treatment (Figure 4.3a). Additionally, quantitative real-time PCR indicated a significant upregulation of genes associated with motility, motAB (2.57 fold), as well as chemotaxis genes che $A$ (3.71 fold) and cheW (2.84 fold) under 30mM SCFA relative to 172mM SCFA treatment (Figure 4.3b). 


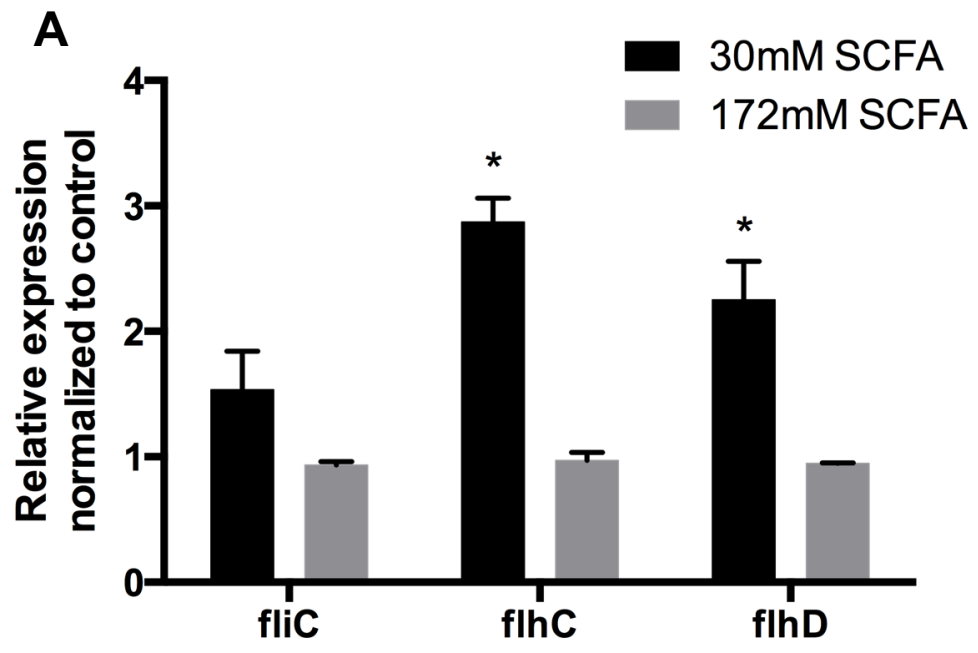

B

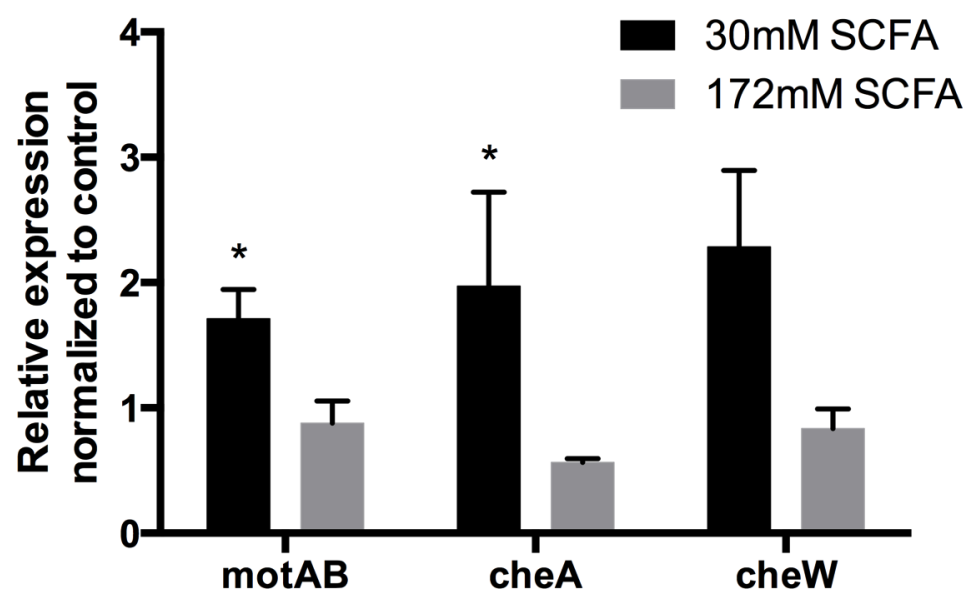

Figure 4.3: Gene expression analysis following SCFA mix treatment of EHEC O157:H7. Quantitative real-time PCR analysis of (a) $f l i C$, flhC, and $f l h D$; and (b) motAB, cheA, and cheW after either 30mM or $172 \mathrm{mM} \mathrm{SCFA} \mathrm{stress} \mathrm{for} 6 \mathrm{hr}$ at $37^{\circ} \mathrm{C}+5 \% \mathrm{CO}_{2}$, normalized to gapA. Data bars represent means $\pm \mathrm{SEMs}$, $N=3$. * indicates a significant difference from 172mM SCFA treatment, $P<0.05$ by two-way ANOVA.

\subsubsection{FliC protein expression is upregulated in response to $30 \mathrm{mM} \mathrm{SCFA}$}

Since the transcriptional analysis revealed differential expression of flagellar genes in response to either low or high SCFA treatments, we then assessed FliC protein expression in response to each treatment. Immunoblot analysis confirmed a strong, significant 3.7 fold increase 
of FliC expression following 30mM SCFA treatment relative to $172 \mathrm{mM}$ SCFA treatment (Figure 4.4). When compared to their respective sodium chloride controls, immunoblot analysis of FliC expression showed a significant 4.8 fold increase for $30 \mathrm{mM}$ SCFA relative to its $30 \mathrm{mM} \mathrm{NaCl}$ control, and a small 1.3 fold upregulation for $172 \mathrm{mM}$ SCFA relative to its $172 \mathrm{mM} \mathrm{NaCl}$ control, indicating that flagella expression is not responding to osmolarity changes alone.

A

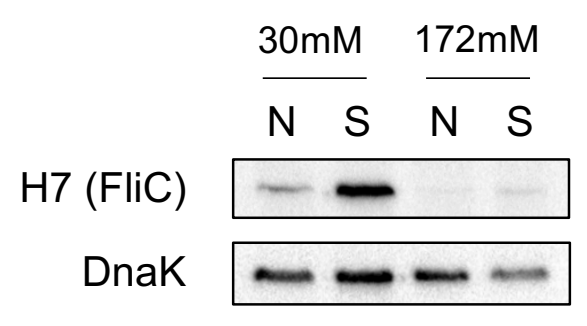

B

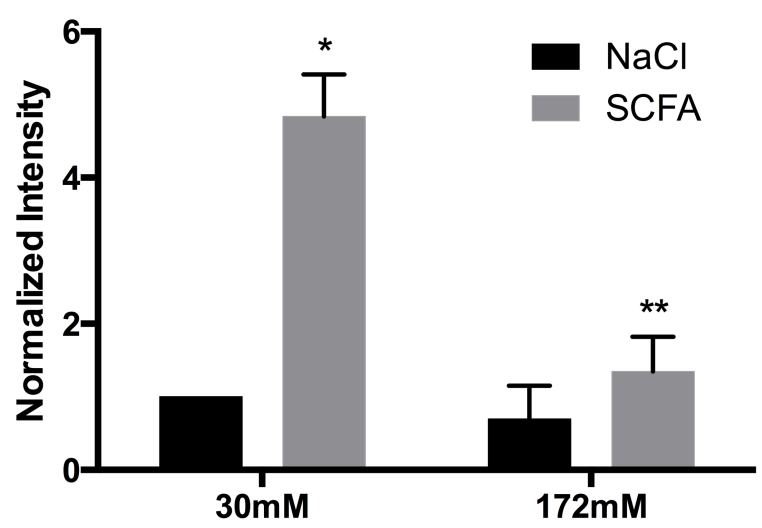

Figure 4.4: Immunoblot analysis of FliC (H7) protein expression following SCFA mix treatment of EHEC 0157:H7. (a) ECL-detected blot images of FliC (H7) after either 30mM or 172mM SCFA stress for $6 \mathrm{hr}$ at $37^{\circ} \mathrm{C}+5 \% \mathrm{CO}_{2}$. (b) Quantified protein expression from (a), normalized to DnaK (loading control). Data bars represent means \pm SEMs, $N=3$. * indicates a significant difference from corresponding $\mathrm{NaCl}$ control; $* *$ indicates a significant difference from 30mM SCFA treatment, $P<0.05$ by two-way ANOVA.

Additionally, we examined flagella expression using immunofluorescence microscopy of SCFA-stressed EHEC stained with anti-H7 (FliC) and an Alexa Fluor 488-conjugated secondary antibody. Consistent with the immunoblot analysis, baseline levels of flagella were seen in the $30 \mathrm{mM} \mathrm{NaCl}$ treated sample with a strong increase in flagella expression in the $30 \mathrm{mM} \mathrm{SCFA}$ treated sample (Figure 4.5). In contrast, no visible flagella were detected in either of the $172 \mathrm{mM}$ $\mathrm{NaCl}$ control or $172 \mathrm{mM}$ SCFA treated sample groups. These results are consistent with the DNA 
microarray, qRT-PCR, and immunoblot analyses which suggest upregulation of FliC expression after exposure to $30 \mathrm{mM}$ SCFA and downregulation of FliC expression following exposure to $172 \mathrm{mM}$ SCFA.

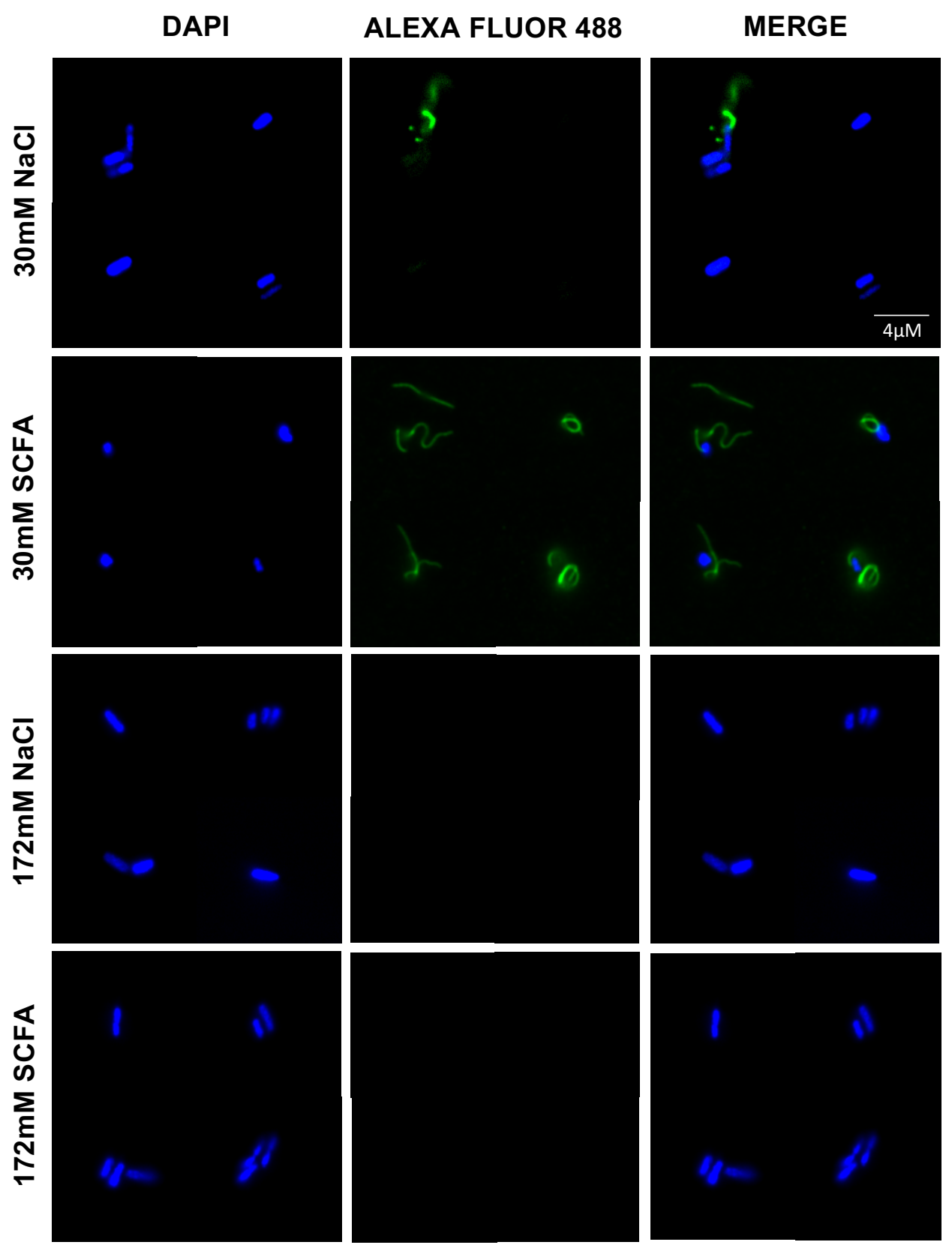

Figure 4.5: Immunofluorescence analysis of flagella expression following SCFA mix treatment of EHEC O157:H7. Immunofluorescence staining of flagella after either 30mM or 172mM SCFA stress for $6 \mathrm{hr}$ at $37^{\circ} \mathrm{C}+5 \% \mathrm{CO}_{2}$. Flagella were stained with anti-H7 (FliC) followed by an AlexaFluor-488 conjugated secondary antibody and DAPI. 


\subsubsection{EHEC motility is modulated in response to $30 \mathrm{mM}$ and $172 \mathrm{mM}$ SCFA treatment}

We then assessed the impact of differential SCFA treatment on functional motility of EHEC O157:H7, as seen by soft-agar motility halos. Treatment with 30mM SCFA showed a significant increase in motility relative to the $30 \mathrm{mM} \mathrm{NaCl}$ control, consistent with the increase in expression of FliC seen under the same treatment (Figure 4.6). Similarly, treatment with $172 \mathrm{mM}$ SCFA showed a significant decrease in motility relative to both its $172 \mathrm{mM} \mathrm{NaCl}$ control and to the 30mM SCFA treatment, consistent with the decrease in FliC expression seen under the same treatment. Taken together, these results suggest that flagella expression is directly proportional to motility, where an increase in flagella expression is associated with an increase in motility and vice versa.
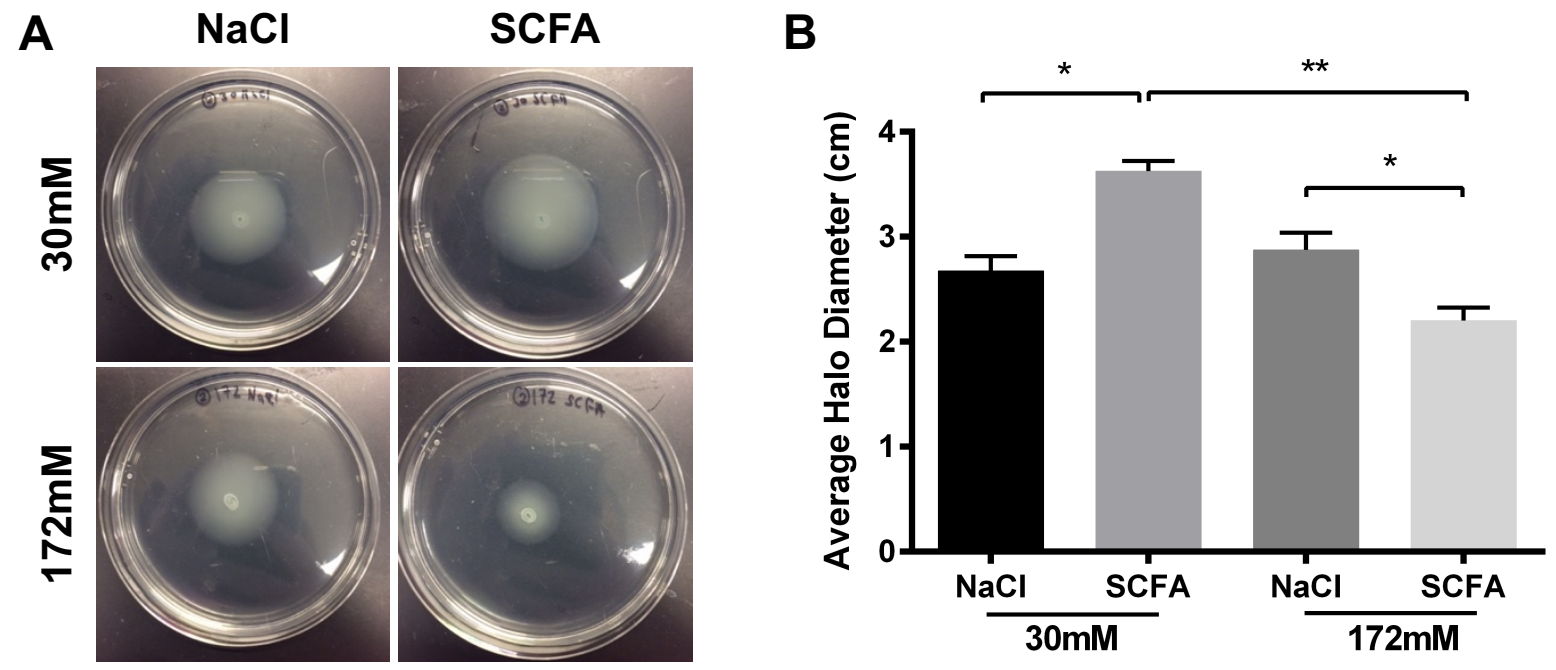

Figure 4.6: Soft-agar motility analysis following SCFA mix treatment of EHEC O157:H7 $7^{\ddagger}$. (a) Representative images of motility halos after either $30 \mathrm{mM}$ or $172 \mathrm{mM}$ SCFA stress for $6 \mathrm{hr}$ at $37^{\circ} \mathrm{C}+5 \%$ $\mathrm{CO}_{2}$. (b) Quantified average halo diameter from a. Data bars represent means \pm SEMs, $N=3 . *$ indicates a significant difference from respective $\mathrm{NaCl}$ controls; $* *$ indicates a significant difference from $30 \mathrm{mM}$ SCFA treatment, $P<0.05$ by two-way ANOVA. ${ }^{\ddagger}$ The motility data presented was carried out in conjunction with JeeIn Kim (Ryerson University, Toronto, ON). 


\subsubsection{Impact of individual SCFAs on flagella expression of EHEC}

Since varying concentrations of the SCFA mix treatment resulted in differential expression of FliC, we next assessed the impact of the individual components (acetate, butyrate, and propionate) on FliC expression. The individual SCFAs were either set to their original concentration within the SCFA mix or set to $30 \mathrm{mM}$ or $172 \mathrm{mM}$ in order to determine if they were able to induce a similar level of expression as the SCFA mix at $30 \mathrm{mM}$ or $172 \mathrm{mM}$. When the individual components were set to their original concentrations within the 30mM SCFA mix, they each elicited a significant increase in FliC expression relative to the $30 \mathrm{mM} \mathrm{NaCl}$ control, similar to that shown by the 30mM SCFA mix (Figure 4.7a-b). When acetate and propionate were set to their original concentrations within the $172 \mathrm{mM}$ SCFA mix, they each induced a similar small increase in FliC expression as the $172 \mathrm{mM}$ SCFA mix relative to its $\mathrm{NaCl}$ control. Interestingly, when butyrate was set to its original concentration within the $172 \mathrm{mM}$ SCFA mix, it elicited a strong significant increase in FliC expression relative to the $172 \mathrm{mM} \mathrm{NaCl}$ control, unlike the 172mM SCFA mix (Figure 4.7a-b), suggesting that butyrate may be acting in a pathway independent of acetate and propionate to regulate flagella expression.

To confirm this, we tested the impact of the individual SCFAs all set to either $30 \mathrm{mM}$ or $172 \mathrm{mM}$. When acetate, butyrate, and propionate were set to $30 \mathrm{mM}$, they each induced a significant increase in FliC expression similar to that of the 30mM SCFA mix (Figure 4.7c-d). Similarly, when acetate, butyrate, and propionate were set to $172 \mathrm{mM}$, they each induced a similar low level of FliC expression as the $172 \mathrm{mM}$ SCFA mix, suggesting that the upregulation of FliC may be limited to only low concentrations of the individual SCFAs. 
A
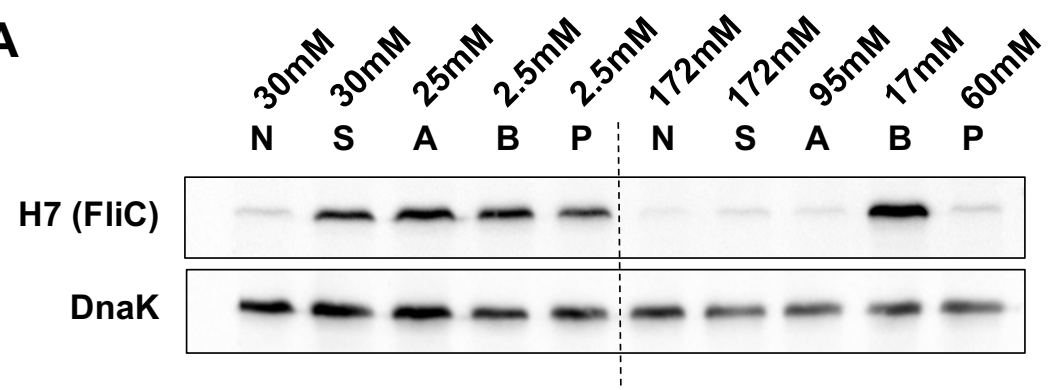

B

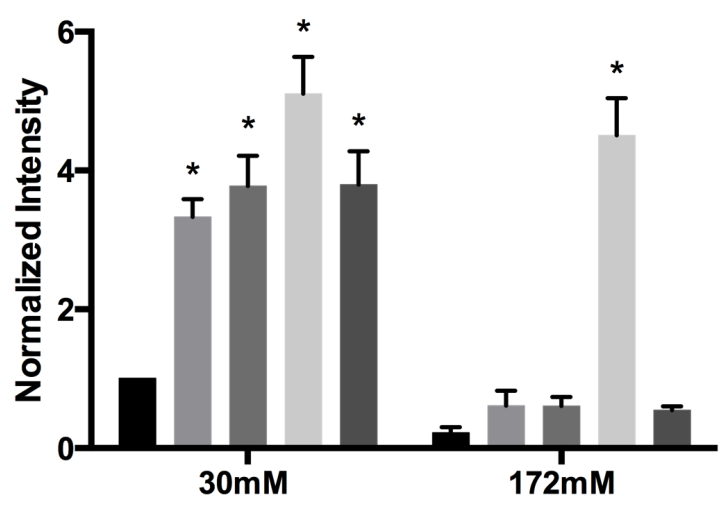

$\mathrm{NaCl}$

- SCFA

- Acetate

- Butyrate

- Propionate

C

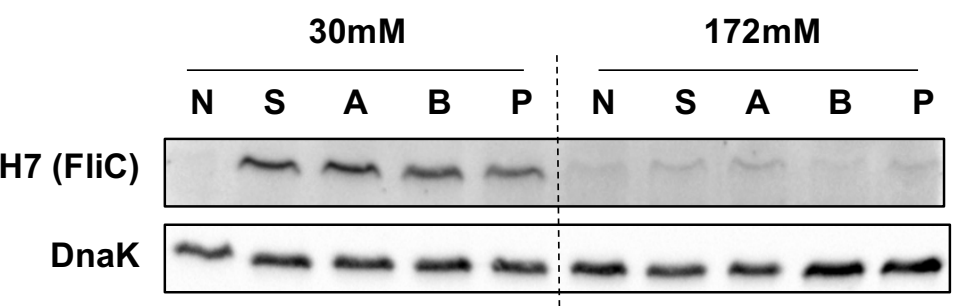

D

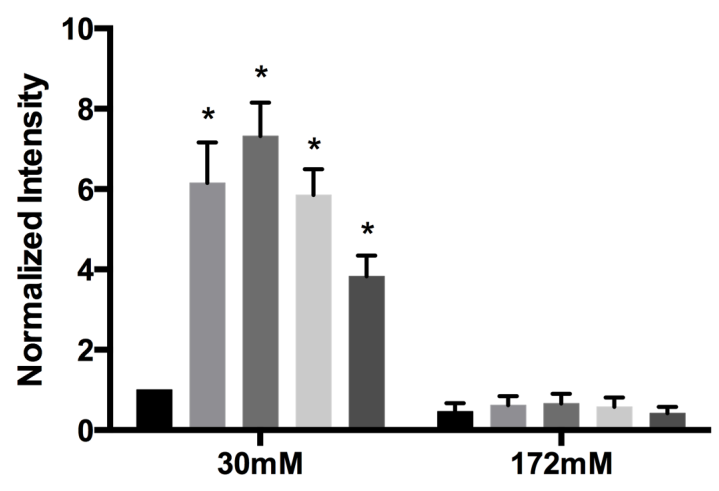

$\mathrm{NaCl}$

SCFA

Acetate

Butyrate

Propionate 
Figure 4.7: Immunoblot analysis of FliC (H7) protein expression following individual SCFA treatment of EHEC O157:H7. (a) ECL-detected blot images of FliC (H7) after individual SCFA treatment, set to original concentration within SCFA mix, for $6 \mathrm{hr}$ at $37^{\circ} \mathrm{C}+5 \% \mathrm{CO}_{2}(\mathrm{~N}-\mathrm{NaCl} ; \mathrm{S}-\mathrm{SCFA}$ mix; A - acetate; B - butyrate; P - propionate). (b) Quantified protein expression from a, normalized to DnaK (loading control). (c) ECL-detected blot images of FliC (H7) after individual SCFA treatment, set to either 30mM or $172 \mathrm{mM}$. (d) Quantified protein expression from a, normalized to DnaK. Data bars represent means \pm SEMs, $N=3{ }^{*}$ indicates a significant difference from corresponding $\mathrm{NaCl}$ control, $P<0.05$ by two-way ANOVA.

With both the western blot and fluorescent microscopy results, there was a modest level of FliC expression in the $30 \mathrm{mM} \mathrm{NaCl}$ control and virtually none in the $172 \mathrm{mM} \mathrm{NaCl}$ control. However, FliC expression under 30mM SCFA treatment was always significantly higher than either the $30 \mathrm{mM}$ or $172 \mathrm{mM} \mathrm{NaCl}$ controls. While the contribution of an osmolarity effect cannot be fully ruled out, particularly at the higher SCFA mix concentration, collectively the data suggest that osmolarity is not the defining factor in FliC expression.

To test whether the individual SCFAs caused upregulation of FliC at different concentration ranges, we tested the impact of a concentration gradient $(0-170 \mathrm{mM})$ of acetate, butyrate, and propionate on FliC expression. For all 3 individual SCFAs, FliC expression peaked at $30 \mathrm{mM}$ and had a sharp decline in expression at $60 \mathrm{mM}$, which remained consistent through to 170mM (Figure 4.8). Together these results demonstrate that while acetate, butyrate, and propionate may be acting through independent pathways, they cause a similar level of FliC expression at all concentrations tested. 

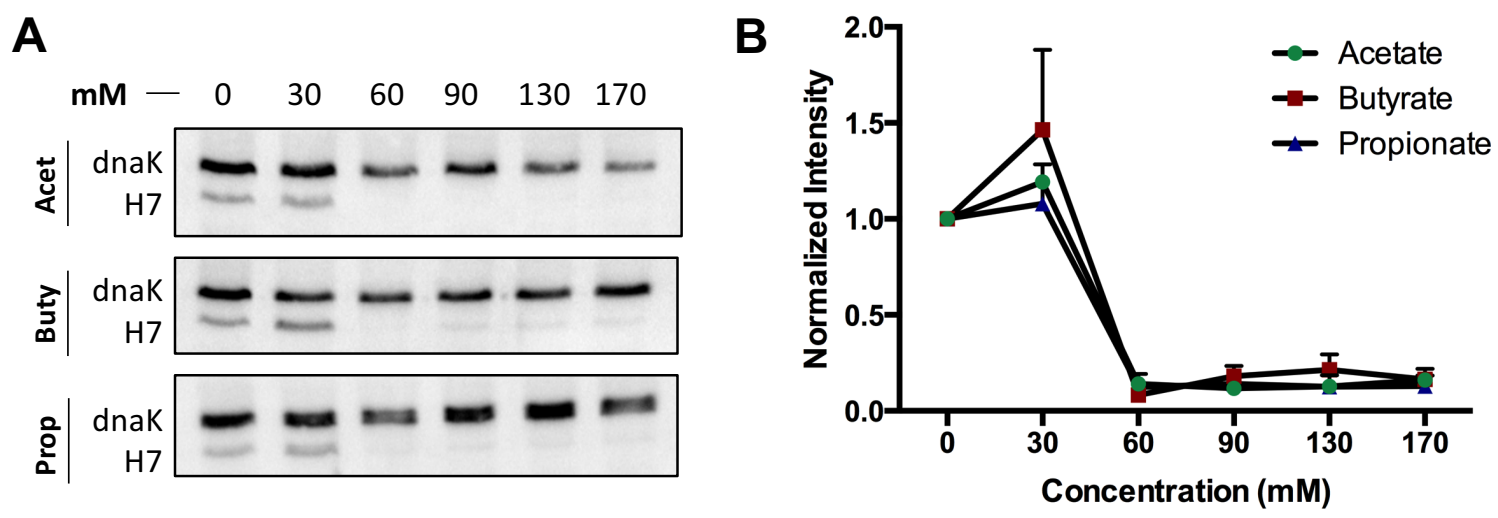

Figure 4.8: Immunoblot analysis of FliC $(\mathrm{H} 7)$ protein expression following a concentration gradient of individual SCFA treatment of EHEC O157:H7. (a) ECL-detected blot images of FliC (H7) after treatment with a concentration gradient of acetate, butyrate, and propionate for $6 \mathrm{hr}$ at $37^{\circ} \mathrm{C}+5 \% \mathrm{CO}_{2}$. (b) Quantified protein expression from a, normalized to DnaK (loading control). Data points represent means \pm SEMs, $N=3$.

\subsection{DISCUSSION}

This study shows the differential modulation of EHEC flagella in response to physiologically relevant short chain fatty acid mixtures typical of those encountered during transit through the human GI tract. The findings of this study suggest that EHEC utilizes the changing SCFA concentrations as an environmental cue to determine its location along the GI tract, and consequently modulate numerous virulence genes including flagella and motility genes.

When EHEC is exposed to lower concentrations of a SCFA mix, representative of the small intestinal conditions, flagellar genes are significantly upregulated. Immunoblot and immunofluorescence analysis confirm the upregulated expression of $\mathrm{H} 7$ flagella and the main structural protein, FliC, under these low SCFA mix conditions. The motility data subsequently confirmed that the upregulation of flagella at low SCFA concentrations is correlated with a 
significant increase in EHEC motility. Consequently, when EHEC is exposed to higher concentrations of a SCFA mix, representative of the large intestinal conditions, flagellar genes are significantly downregulated. The immunoblot and immunofluorescence analysis further confirm these results for the $\mathrm{H} 7$ flagella and FliC protein. Moreover, the motility data supports the correlation between the downregulation of flagella at high SCFA concentrations and a decrease in EHEC motility. Together, these findings support a model in which exposure to low concentrations of SCFAs cues EHEC to upregulate flagella and ensure functional motility during transit through the small intestine, whereas exposure to high concentrations of SCFAs cues EHEC to downregulate flagella and motility as EHEC nears its preferred site of colonization in the distal colon (Bannas et al., 2013; Lewis et al., 2015).

Although the relative levels and composition of SCFA mixes in the lumen of specific regions along the human GI tract are broad and dynamic over time, there appears to be a consensus that SCFA levels are lowest in the small intestine, ranging from approximately 20 to $40 \mathrm{mM}$, increasing dramatically in the cecum and colon to concentrations ranging between 160 to $200 \mathrm{mM}$, and then declining through the descending and sigmoidal regions of the large intestine to concentrations between 70 to $85 \mathrm{mM}$ (Herold et al., 2009; Lawhon et al., 2003; Macfarlane et al., 1992). In this study, we chose $30 \mathrm{mM}$ total SCFA concentration as representative of the small intestine and $172 \mathrm{mM}$ total SCFA concentrations as representative of the large intestine, and most importantly, close to that typically encountered in the specific site of colonization. Our findings, which demonstrate a significant downregulation of flagella expression and motility under this higher SCFA mix, may suggest a switch from EHEC's swimming motility to adhesion of the host epithelium. Motility and host cell adhesion fulfill antagonistic functions, and pathogens often 
employ mechanisms to reciprocally regulate these functions (Akerley et al., 1995; Allison et al., 2012; Li et al., 2001; Morgan et al., 2013).

Changes in oxygen concentration have also been reported to modulate EHEC motility and adhesion, with increased motility associated with a microaerophilic environment similar to that encountered within the mucosal epithelial layer (Schüller and Phillips, 2010). Since the focus of this study was to understand the role of changing concentrations of SCFAs alone on flagella and motility modulation, we chose to examine motility and flagella expression under static with 5\% $\mathrm{CO}_{2}$ conditions rather than either microaerophilic or shaking culture conditions. Static conditions have traditionally been used in studies of virulence factor expression in EHEC, and have been suggested to roughly mimic the oxygen concentration within the intestinal lumen (Yin et al., 2011).

This study also examined the role of specific SCFAs (acetate, butyrate, and propionate) in modulation of flagella expression. The findings suggested that when the individual SCFAs are each set to a concentration of $172 \mathrm{mM}$, these conditions each elicit an equal upregulation of the $\mathrm{H} 7$ flagella protein, FliC. Interestingly, we found that the $\mathrm{FliC}$ expression under $30 \mathrm{mM}$ acetate matched that of the $30 \mathrm{mM}$ SCFA mix while treatment with $30 \mathrm{mM}$ propionate resulted in slightly lower FliC expression, suggesting that the response to acetate may be more important in contributing to the overall response to the SCFA mix. However, when a concentration gradient of each SCFA between 0 to $170 \mathrm{mM}$ was tested, all three demonstrated the same trend in expression, with a peak in flagella expression at $30 \mathrm{mM}$ and a significant downregulation from 60 to $170 \mathrm{mM}$. Several other studies have examined the role of specific SCFAs on EHEC virulence including motility. Momose et al reported that any of acetate (12 to $72 \mathrm{mM})$, propionate ( 7 to $42 \mathrm{mM}$ ), or acetate plus propionate $(12: 7,24: 14$, or $36: 21)$ all reduced EHEC motility under anaerobic conditions (Momose et al., 2008). Additionally, Takao et al reported that when exposed to 20mM 
butyrate, similar to that encountered in the large intestine, EHEC showed increased flagellar gene expression (Takao et al., 2014). Interestingly, this response was specific to butyrate and not seen with either acetate or propionate, leading the authors to conclude that butyrate at $20 \mathrm{mM}$ concentration played a key role in the upregulation of EHEC motility. It is of interest to note that the concentration of butyrate present within the $172 \mathrm{mM}$ SCFA mix used in this study is $17 \mathrm{mM}$, and we also observed a significant increase in FliC expression under $17 \mathrm{mM}$ butyrate treatment alone. However, when combined within the total mix of SCFAs representative of the large intestine, that increase was not observed and a decrease in flagellar expression was seen relative to that seen under the $30 \mathrm{mM}$ SCFA mix.

Motility provides EHEC with a profound advantage during transit through the human GI tract, permitting the pathogen to avoid detrimental locations and to find more favourable niches. Consequently, microenvironmental stressors along the GI tract can act as cues that modulate EHEC virulence and motility, and may play a vital role in its pathogenesis. Together, the findings

of this study demonstrate that differing concentrations of SCFA mixes elicit differential modulation of EHEC motility and flagella expression, with higher expression seen at small intestinal-like SCFA mixes and decreased expression seen under large intestinal-like SCFA mixes.

\subsection{CONCLUSIONS \& FUTURE DIRECTIONS}

\subsubsection{Conclusions}

In summary, we have successfully demonstrated that low and high concentrations of SCFA mixes representative of transit through the small and large intestines respectively differentially modulates flagella and motility, a critical component of EHEC's virulence. At lower SCFA concentrations, representative of transit through the small intestine, flagella expression was 
increased at both the transcriptional and translational level. A similar increase in motility was also observed at these low SCFA concentrations. However, at higher SCFA concentrations, representative of transit through the large intestine, flagella expression and subsequent motility was decreased. Since motility is a vital aspect of a pathogen's ability to transit through the host GI tract, thereby permitting the pathogen to avoid detrimental locations and to find more favourable niches, the results of this study suggest that SCFAs are acting as an environmental cue for EHEC relative to its colonization site in the GI tract. Moreover, a model could be envisioned in which SCFA mixes typical of the small intestine, stimulates increased flagella expression to promote motility toward the colonization site in the large intestine (Figure 4.9). Conversely, when EHEC enters the large intestine and senses the high concentrations of SCFAs, it downregulates flagella expression as it nears the preferred site of colonization. This change may promote EHEC adhesion to the host epithelium in the caecum and colon and infect the host.

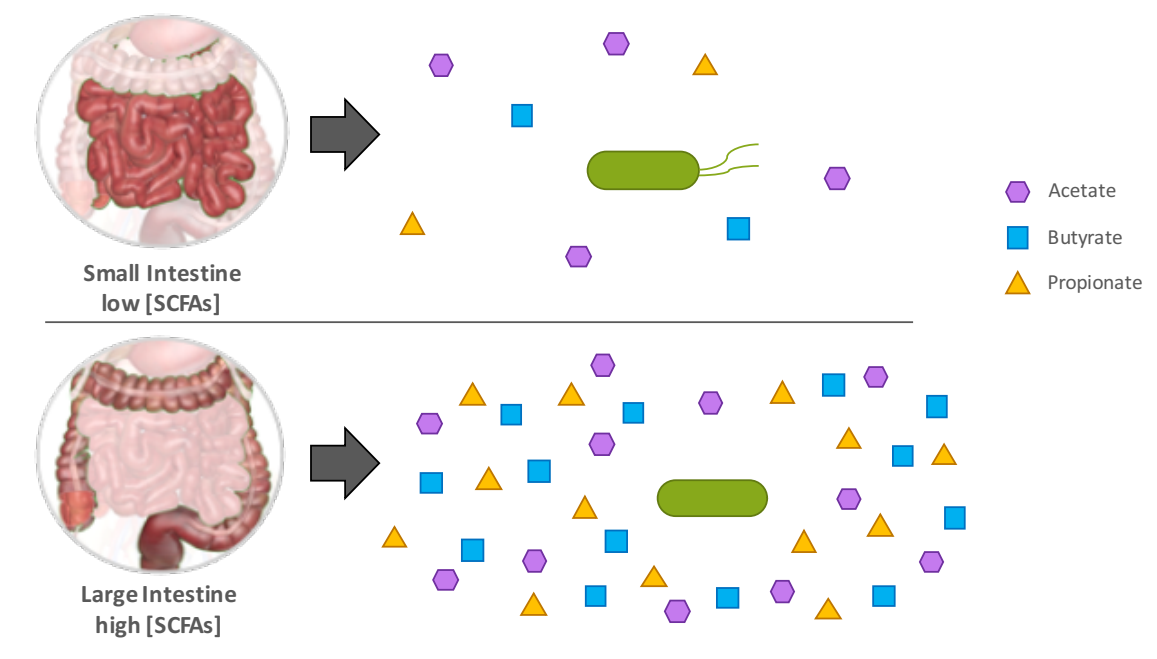

Figure 4.9: Model of the differential modulation of flagella expression by varying concentrations of short chain fatty acids. In the proposed model, low concentrations of SCFAs in the small intestines upregulate flagella to promote motility to the large intestine. Once in the large intestine at the site of colonization, high concentrations of SCFAs downregulate flagella to decrease motility and promote adherence to the host epithelium in order to effectively infect the host. 


\subsubsection{Future Directions \& Significance}

While this study indicates that microenvironmental stresses within the human GI tract, such as SCFAs, may act as chemical cues to modulate EHEC virulence in a way that could promote infection of the host, the exact mechanisms by which SCFAs modulate virulence is still under investigation. Our lab has recently identified several two-component systems in E. coli K12 that may play a role in SCFA-induced modulation of EHEC virulence (Kim, 2016, unpublished Master's thesis). A deeper understanding of how these two-component systems sense SCFAs, and if different two-component systems respond to different individual SCFAs is necessary. Furthermore, in order to fully understand how SCFAs are sensed and cause flagella modulation, it is vital to determine what other proteins are involved in this pathway. Aside from understanding the mechanism by which SCFAs are sensed by EHEC, analyzing how different SCFA compositions affect virulence modulation is also critical for downstream applications. More specifically, since the breakdown of different dietary fibers results in differing SCFA compositions, a more thorough understanding of how different SCFA compositions affect virulence modulation can be exploited as a means to protect the host against infection susceptibility. Additionally, since the composition of the gut microbiota results in differing SCFA compositions, a more thorough understanding of how the microbiota can be manipulated to produce SCFA compositions which do not significantly enhance EHEC virulence would be most benefecial. For example, the use of probiotics or prebiotics may have a profound impact on the SCFA compositions produced through fermentation of dietary fiber, and in turn impact the modulation of EHEC virulence. 
In conclusion, this research sheds light on the fact that differing concentrations and compositions of SCFAs act as an environmental cue to modulate EHEC virulence to promote successful infection of the host. Moreover, since SCFAs are a biproduct of dietary fiber, the findings of this research have significant implications for how humans can manipulate their risk of being infected through changes to their diet and microbiota composition. More specifically, by changing our diets in such a way that optimizes our GI tract SCFA concentration and composition, and subsequently the composition of our GI microbiota, we may be able to enhance our resistance to EHEC infection. 


\section{CONCLUSIONS \& SIGNIFICANCE}


The clinically relevant foodborne pathogen enterohemorrhagic Escherichia coli (EHEC) remains a global concern, with new outbreaks seen each year. Consequently, there is an increased interest in the development of novel therapeutic strategies, in particular prevention strategies which target the pathogen prior to colonization of the human large intestine. Interestingly, as EHEC navigates through the human GI tract to its preferred site of colonization, the pathogen faces a multitude of host assaults that act as an antimicrobial barrier. Although exposure to these innate host defenses poses a significant threat to the survival of EHEC, the bacteria have evolved mechanisms to not only survive these stresses but also use them as cues to modulate its virulence so as to that enhances its potential to successfully infect its host. The research carried out in this doctoral dissertation aimed to understand the response of EHEC O157:H7 to these human gastrointestinal chemical signals, in particular gastric acid stress in the stomach and short chain fatty acid stress in the large intestine, and to use this understanding to develop novel antimicrobial strategies.

The first objective of this study focussed on the use of a novel antimicrobial peptide wrwycr as a prevention strategy to enhance acid-induced killing of EHEC, a result of increased DNA damage inflicted by exposure to gastric acid in the human stomach. Using the $C$. rodentium mouse model of EHEC infection, our findings demonstrated that brief peptide pre-treatment of $C$. rodentium prior to infection ameliorated infection outcomes and reduced colonic epithelial cell hyperplasia, a hallmark of $C$. rodentium infection, in a dose-dependent manner with the minimum effective concentration tested being $50 \mu \mathrm{M}$. Moreover, we successfully showed that neither administration of the peptide alone nor infection with peptide pre-treated $C$. rodentium induced alterations in the gut microbiome as determined by fecal microbial composition analysis. Taken together the results indicate that the peptide-induced killing is specific to the pathogen which is 
initially pre-treated with the effective dose of the peptide. These results also demonstrate that DNA damage induced by acute acid exposure, similar to that encountered during gastric passage, may be potentiated by brief pre-treatment with the antimicrobial peptide wrwycr. The combination of peptide-acid treatment enhances bacterial cell death, thereby effectively killing the pathogen prior to entering the lower GI tract.

In the second objective of this study, we aimed to identify a connection between two distinct effects of the peptide wrwycr on bacterial cells - (1) its role in enhancing acid-induced killing through inhibition of DNA repair mechanisms, and (2) its role in causing an increase in chelatable iron within the bacterial cell, likely due to the breakdown of iron-sulfur clusters - by exploring the role of the DNA-binding protein, Dps, on peptide- and peptide-acid- induced EHEC killing. Our findings demonstrate a key role for Dps in protecting EHEC against acid, peptide, and peptide-acid treatment. Specifically, we showed that when $d p s$ is disrupted, there is significantly increased levels of bacterial cell death upon exposure to either acid, peptide, or peptide-acid treatment. This decrease in survival is restored when $d p s$ is re-inserted on a vector plasmid. Our findings also demonstrate that Dps protects EHEC against these stresses in part by protecting against the formation of hydroxyl radicals, likely due to the peptide-induced increase in chelatable iron which drives the Fenton reaction. These findings suggest that Dps may provide a key link between the two distinct effects of this antimicrobial peptide on EHEC.

The third and final obbjective of this study examined how EHEC differentially modulates its virulence, and specifically its motility, in response to changing concentrations of a SCFA mix containing acetate, butyrate, and propionate. We examined the response of EHEC flagella expression and motility to a low concentration of SCFA mix (30mM), representative of transit through the small intestine, and to a high concentration of SCFA mix (172mM), representative of 
transit through the large intestine. Our findings demonstrate that exposure to low concentrations of a SCFA mix dramatically upregulated H7 flagella protein, FliC, expression and significantly upregulated motility, consistent with the model that EHEC upregulates motility during transit through the small intestine in order to reach its site of colonization in the large intestine. In contrast, when EHEC is exposed to high concentrations of a SCFA mix, both flagella expression and motility were significantly downregulated, suggesting that motility is less important once at the site of colonization, and possibly the function of existing flagella may in fact be switched for use in initial adhesion to the host epithelial cells. These findings reveal a key mechanism by which EHEC modulates its virulence in response to changing concentrations and compositions of SCFAs.

Taken together, the findings of this study reveal how EHEC has adapted and evolved mechanisms to not only survive the multitude of host defenses encountered during transit through the human GI tract, but also use these stressors as environmental cues within the GI tract to modulate virulence in a way that increases its potential to successfully infect the host. Interestingly, these results also demonstrate a unique strategy for preventing EHEC infection prior to colonization, by exploiting the innate host defenses along the GI tract. The use of the antimicrobial peptide wrwycr to potentiate acid-induced bacterial cell death presents a key model in which small antimicrobial peptides can be used to enhance the innate host defenses present throughout the human GI tract, such as bile salts and CAMPs, thereby potentiating the detrimental effects of these host defenses to increase bacterial cell death. This novel strategy demonstrates the potential to reduce the risk of increased disease risk as seen with traditional antibiotic treatments, by effectively targeting the pathogen prior to infection of the host. Moreover, this strategy may be applied to other pathogens, providing a successful means of preventing enteric infection by enhancing the natural host assaults present along the human GI tract. 


\section{REFERENCES}


Abe, H., Tatsuno, I., Tobe, T., Okutani, A., Sasakawa, C., 2002. Bicarbonate ion stimulates the expression of locus of enterocyte effacement-encoded genes in enterohemorrhagic Escherichia coli O157:H7. Infect. Immun. 70, 3500-3509.

Afset, J.E., Bevanger, L., Romundstad, P., Bergh, K., 2004. Association of atypical enteropathogenic Escherichia coli (EPEC) with prolonged diarrhoea. J. Med. Microbiol. 53, 1137-1144. doi:10.1099/jmm.0.45719-0

Akerley, B.J., Cotter, P.A., Miller, J.F., 1995. Ectopic expression of the flagellar regulon alters development of the bordetella-host interaction. Cell 80, 611-620. doi:10.1016/00928674(95)90515-4

Ali Azam, T., Iwata, A., Nishimura, A., Ueda, S., Ishihama, A., 1999. Growth phase-dependent variation in protein composition of the Escherichia coli nucleoid. J. Bacteriol. 181, 63616370.

Allison, S.E., Silphaduang, U., Mascarenhas, M., Konczy, P., Quan, Q., Karmali, M., Coombes, B.K., 2012. Novel repressor of Escherichia coli O157:H7 motility encoded in the putative fimbrial cluster OI-1. J. Bacteriol. 194, 5343-5352. doi:10.1128/JB.01025-12

Almirón, M., Link, A.J., Furlong, D., Kolter, R., 1992. A novel DNA-binding protein with regulatory and protective roles in starved Escherichia coli. Genes Dev. 6, 2646-2654.

Altuvia, S., Almirón, M., Huisman, G., Kolter, R., Storz, G., 1994. The dps promoter is activated by OxyR during growth and by IHF and sigma S in stationary phase. Mol. Microbiol. 13, $265-272$.

Arbeloa, A., Blanco, M., Moreira, F.C., Bulgin, R., Lopez, C., Dahbi, G., Blanco, J.E., Mora, A., Alonso, M.P., Mamani, R.C., Gomes, T.A.T., Blanco, J., Frankel, G., 2009. Distribution of espM and espT among enteropathogenic and enterohaemorrhagic Escherichia coli. J. Med. Microbiol. 58, 988-995. doi:10.1099/jmm.0.010231-0

Audia, J.P., Webb, C.C., Foster, J.W., 2001. Breaking through the acid barrier: an orchestrated response to proton stress by enteric bacteria. Int. J. Med. Microbiol. IJMM 291, 97-106. doi:10.1078/1438-4221-00106

Babitzke, P., Romeo, T., 2007. CsrB sRNA family: sequestration of RNA-binding regulatory proteins. Curr. Opin. Microbiol. 10, 156-163. doi:10.1016/j.mib.2007.03.007

Bannas, P., Fraedrich, K., Treszl, A., Bley, T., Herrmann, J., Habermann, C., Derlin, T., Henes, F., Wenzel, U., Adam, G., Yamamura, J., 2013. Shiga Toxin-Producing E. Coli O104:H4 
Outbreak 2011 in Germany: Radiological Features of Enterohemorrhagic Colitis. RöFo Fortschritte Auf Dem Geb. Röntgenstrahlen Bildgeb. Verfahr. 185, 434-439. doi:10.1055/s-0032-1330520

Barnett Foster, D., 2013. Modulation of the enterohemorrhagic E. coli virulence program through the human gastrointestinal tract. Virulence 4, 315-323. doi:10.4161/viru.24318

Battesti, A., Majdalani, N., Gottesman, S., 2011. The RpoS-Mediated General Stress Response in Escherichia coli ${ }^{*}$. Annu. Rev. Microbiol. 65, 189-213. doi:10.1146/annurev-micro090110-102946

Beltrametti, F., Kresse, A.U., Guzmán, C.A., 1999. Transcriptional regulation of the esp genes of enterohemorrhagic Escherichia coli. J. Bacteriol. 181, 3409-3418.

Bergman, E.N., 1990. Energy contributions of volatile fatty acids from the gastrointestinal tract in various species. Physiol. Rev. 70, 567-590.

Bhavsar, A.P., Guttman, J.A., Finlay, B.B., 2007. Manipulation of host-cell pathways by bacterial pathogens. Nature 449, 827-834. doi:10.1038/nature06247

Binder, H.J., 2010. Role of colonic short-chain fatty acid transport in diarrhea. Annu. Rev. Physiol. 72, 297-313. doi:10.1146/annurev-physiol-021909-135817

Bingham, S.A., Day, N.E., Luben, R., Ferrari, P., Slimani, N., Norat, T., Clavel-Chapelon, F., Kesse, E., Nieters, A., Boeing, H., Tjønneland, A., Overvad, K., Martinez, C., Dorronsoro, M., Gonzalez, C.A., Key, T.J., Trichopoulou, A., Naska, A., Vineis, P., Tumino, R., Krogh, V., Bueno-de-Mesquita, H.B., Peeters, P.H.M., Berglund, G., Hallmans, G., Lund, E., Skeie, G., Kaaks, R., Riboli, E., European Prospective Investigation into Cancer and Nutrition, 2003. Dietary fibre in food and protection against colorectal cancer in the European Prospective Investigation into Cancer and Nutrition (EPIC): an observational study. Lancet Lond. Engl. 361, 1496-1501.

Black, P.N., DiRusso, C.C., 1994. Molecular and biochemical analyses of fatty acid transport, metabolism, and gene regulation in Escherichia coli. Biochim. Biophys. Acta 1210, $123-$ 145.

Boldt, J.L., Pinilla, C., Segall, A.M., 2004. Reversible inhibitors of lambda integrase-mediated recombination efficiently trap Holliday junction intermediates and form the basis of a novel assay for junction resolution. J. Biol. Chem. 279, 3472-3483.

doi:10.1074/jbc.M309361200 
Borenshtein, D., McBee, M.E., Schauer, D.B., 2008. Utility of the Citrobacter rodentium infection model in laboratory mice. Curr. Opin. Gastroenterol. 24, 32-37. doi:10.1097/MOG.0b013e3282f2b0fb

Borenshtein, D., Schlieper, K.A., Rickman, B.H., Chapman, J.M., Schweinfest, C.W., Fox, J.G., Schauer, D.B., 2009. Decreased Expression of Colonic Slc26a3 and Carbonic Anhydrase IV as a Cause of Fatal Infectious Diarrhea in Mice. Infect. Immun. 77, 3639-3650. doi:10.1128/IAI.00225-09

Brenneman, K.E., Willingham, C., Kilbourne, J.A., Curtiss, R., Roland, K.L., 2014. A low gastric $\mathrm{pH}$ mouse model to evaluate live attenuated bacterial vaccines. PloS One 9, e87411. doi:10.1371/journal.pone.0087411

Bretschneider, G., Berberov, E.M., Moxley, R.A., 2007. Isotype-specific antibody responses against Escherichia coli O157:H7 locus of enterocyte effacement proteins in adult beef cattle following experimental infection. Vet. Immunol. Immunopathol. 118, 229-238. doi:10.1016/j.vetimm.2007.06.005

Brogden, K.A., 2005. Antimicrobial peptides: pore formers or metabolic inhibitors in bacteria? Nat. Rev. Microbiol. 3, 238-250. doi:10.1038/nrmicro1098

Brown, J.L., Ross, T., McMeekin, T.A., Nichols, P.D., 1997. Acid habituation of Escherichia coli and the potential role of cyclopropane fatty acids in low $\mathrm{pH}$ tolerance. Int. J. Food Microbiol. 37, 163-173.

Brown, K.L., Hancock, R.E.W., 2006. Cationic host defense (antimicrobial) peptides. Curr. Opin. Immunol. 18, 24-30. doi:10.1016/j.coi.2005.11.004

Bueris, V., Sircili, M.P., Taddei, C.R., Santos, M.F. dos, Franzolin, M.R., Martinez, M.B., Ferrer, S.R., Barreto, M.L., Trabulsi, L.R., 2007. Detection of diarrheagenic Escherichia coli from children with and without diarrhea in Salvador, Bahia, Brazil. Mem. Inst. Oswaldo Cruz 102, 839-844. doi:10.1590/S0074-02762007005000116

Calhoun, L.N., Kwon, Y.M., 2011. Structure, function and regulation of the DNA-binding protein Dps and its role in acid and oxidative stress resistance in Escherichia coli: a review: Escherichia coli Dps protein. J. Appl. Microbiol. 110, 375-386. doi:10.1111/j.1365-2672.2010.04890.x 
Campellone, K.G., Robbins, D., Leong, J.M., 2004. EspFU is a translocated EHEC effector that interacts with Tir and N-WASP and promotes Nck-independent actin assembly. Dev. Cell 7, 217-228. doi:10.1016/j.devcel.2004.07.004

Castanie-Cornet, M.P., Penfound, T.A., Smith, D., Elliott, J.F., Foster, J.W., 1999. Control of acid resistance in Escherichia coli. J. Bacteriol. 181, 3525-3535.

Ceci, P., Mangiarotti, L., Rivetti, C., Chiancone, E., 2007. The neutrophil-activating Dps protein of Helicobacter pylori, HP-NAP, adopts a mechanism different from Escherichia coli Dps to bind and condense DNA. Nucleic Acids Res. 35, 2247-2256. doi:10.1093/nar/gkm077

Chandrakesan, P., Roy, B., Jakkula, L.U.M.R., Ahmed, I., Ramamoorthy, P., Tawfik, O., Papineni, R., Houchen, C., Anant, S., Umar, S., 2014. Utility of a bacterial infection model to study epithelial-mesenchymal transition, mesenchymal-epithelial transition or tumorigenesis. Oncogene 33, 2639-2654. doi:10.1038/onc.2013.210

Chevance, F.F.V., Hughes, K.T., 2008. Coordinating assembly of a bacterial macromolecular machine. Nat. Rev. Microbiol. 6, 455-465. doi:10.1038/nrmicro1887

Cheville, A.M., Arnold, K.W., Buchrieser, C., Cheng, C.M., Kaspar, C.W., 1996. rpoS regulation of acid, heat, and salt tolerance in Escherichia coli O157:H7. Appl. Environ. Microbiol. 62, 1822-1824.

Chiancone, E., Ceci, P., 2010. The multifaceted capacity of Dps proteins to combat bacterial stress conditions: Detoxification of iron and hydrogen peroxide and DNA binding. Biochim. Biophys. Acta BBA - Gen. Subj. 1800, 798-805. doi:10.1016/j.bbagen.2010.01.013

Choi, S.H., Baumler, D.J., Kaspar, C.W., 2000. Contribution of dps to Acid Stress Tolerance and Oxidative Stress Tolerance in Escherichia coli O157:H7. Appl. Environ. Microbiol. 66, 3911-3916. doi:10.1128/AEM.66.9.3911-3916.2000

Chong, Y., Fitzhenry, R., Heuschkel, R., Torrente, F., Frankel, G., Phillips, A.D., 2007. Human intestinal tissue tropism in Escherichia coli O157 : H7--initial colonization of terminal ileum and Peyer's patches and minimal colonic adhesion ex vivo. Microbiol. Read. Engl. 153, 794-802. doi:10.1099/mic.0.2006/003178-0

Clare, S., John, V., Walker, A.W., Hill, J.L., Abreu-Goodger, C., Hale, C., Goulding, D., Lawley, T.D., Mastroeni, P., Frankel, G., Enright, A.J., Vigorito, E., Dougan, G., 2013. 
Enhanced Susceptibility to Citrobacter rodentium Infection in MicroRNA-155-Deficient Mice. Infect. Immun. 81, 723-732. doi:10.1128/IAI.00969-12

Clarke, M.B., Hughes, D.T., Zhu, C., Boedeker, E.C., Sperandio, V., 2006. The QseC sensor kinase: a bacterial adrenergic receptor. Proc. Natl. Acad. Sci. U. S. A. 103, 10420-10425. doi:10.1073/pnas.0604343103

Collins, J.W., Keeney, K.M., Crepin, V.F., Rathinam, V.A.K., Fitzgerald, K.A., Finlay, B.B., Frankel, G., 2014. Citrobacter rodentium: infection, inflammation and the microbiota. Nat. Rev. Microbiol. 12, 612-623. doi:10.1038/nrmicro3315

Conner, D.E., Kotrola, J.S., 1995. Growth and survival of Escherichia coli O157:H7 under acidic conditions. Appl. Environ. Microbiol. 61, 382-385.

Cook, S.I., Sellin, J.H., 1998. Review article: short chain fatty acids in health and disease. Aliment. Pharmacol. Ther. 12, 499-507.

Croxen, M.A., Finlay, B.B., 2009. Molecular mechanisms of Escherichia coli pathogenicity. Nat. Rev. Microbiol. doi:10.1038/nrmicro2265

Cummings, J.H., 1983. Fermentation in the human large intestine: evidence and implications for health. Lancet Lond. Engl. 1, 1206-1209.

Cummings, J.H., Macfarlane, G.T., 1991. The control and consequences of bacterial fermentation in the human colon. J. Appl. Bacteriol. 70, 443-459.

Cummings, J.H., Pomare, E.W., Branch, W.J., Naylor, C.P., Macfarlane, G.T., 1987. Short chain fatty acids in human large intestine, portal, hepatic and venous blood. Gut 28, 12211227.

Daniell, S.J., Takahashi, N., Wilson, R., Friedberg, D., Rosenshine, I., Booy, F.P., Shaw, R.K., Knutton, S., Frankel, G., Aizawa, S., 2001. The filamentous type III secretion translocon of enteropathogenic Escherichia coli. Cell. Microbiol. 3, 865-871.

Dawson, A.M., Holdsworth, C.D., Webb, J., 1964. Absorption of Short Chain Fatty Acids in Man. Exp. Biol. Med. 117, 97-100. doi:10.3181/00379727-117-29505

Dean-Nystrom, E.A., Bosworth, B.T., Moon, H.W., O’Brien, A.D., 1998. Escherichia coli O157:H7 requires intimin for enteropathogenicity in calves. Infect. Immun. 66, 45604563. 
Deibel, C., Krämer, S., Chakraborty, T., Ebel, F., 1998. EspE, a novel secreted protein of attaching and effacing bacteria, is directly translocated into infected host cells, where it appears as a tyrosine-phosphorylated $90 \mathrm{kDa}$ protein. Mol. Microbiol. 28, 463-474.

Delzenne, N.M., Cani, P.D., 2005. A place for dietary fibre in the management of the metabolic syndrome. Curr. Opin. Clin. Nutr. Metab. Care 8, 636-640.

den Besten, G., van Eunen, K., Groen, A.K., Venema, K., Reijngoud, D.-J., Bakker, B.M., 2013. The role of short-chain fatty acids in the interplay between diet, gut microbiota, and host energy metabolism. J. Lipid Res. 54, 2325-2340. doi:10.1194/jlr.R036012

Dey, M., Patra, S., Su, L.Y., Segall, A.M., 2013. Tumor Cell Death Mediated by Peptides That Recognize Branched Intermediates of DNA Replication and Repair. PLoS ONE 8, e78751. doi:10.1371/journal.pone.0078751

DiRusso, C.C., Black, P.N., Weimar, J.D., 1999. Molecular inroads into the regulation and metabolism of fatty acids, lessons from bacteria. Prog. Lipid Res. 38, 129-197.

Dolgin, E., 2011. As E. coli continues to claim lives, new approaches offer hope. Nat. Med. 17, 755. doi:10.1038/nm0711-755

Donnenberg, M.S., Yu, J., Kaper, J.B., 1993. A second chromosomal gene necessary for intimate attachment of enteropathogenic Escherichia coli to epithelial cells. J. Bacteriol. 175, $4670-4680$.

Donohoe, D.R., Garge, N., Zhang, X., Sun, W., O’Connell, T.M., Bunger, M.K., Bultman, S.J., 2011. The microbiome and butyrate regulate energy metabolism and autophagy in the mammalian colon. Cell Metab. 13, 517-526. doi:10.1016/j.cmet.2011.02.018

Dwyer, D.J., Collins, J.J., Walker, G.C., 2015. Unraveling the Physiological Complexities of Antibiotic Lethality. Annu. Rev. Pharmacol. Toxicol. 55, 313-332. doi:10.1146/annurevpharmtox-010814-124712

Ebel, F., Podzadel, T., Rohde, M., Kresse, A.U., Krämer, S., Deibel, C., Guzmán, C.A., Chakraborty, T., 1998. Initial binding of Shiga toxin-producing Escherichia coli to host cells and subsequent induction of actin rearrangements depend on filamentous EspAcontaining surface appendages. Mol. Microbiol. 30, 147-161.

Echtenkamp, F., Deng, W., Wickham, M.E., Vazquez, A., Puente, J.L., Thanabalasuriar, A., Gruenheid, S., Finlay, B.B., Hardwidge, P.R., 2008. Characterization of the NleF effector 
protein from attaching and effacing bacterial pathogens. FEMS Microbiol. Lett. 281, 98107. doi:10.1111/j.1574-6968.2008.01088.x

e_coli-2.pdf, n.d.

Elliott, S.J., Sperandio, V., Girón, J.A., Shin, S., Mellies, J.L., Wainwright, L., Hutcheson, S.W., McDaniel, T.K., Kaper, J.B., 2000. The locus of enterocyte effacement (LEE)-encoded regulator controls expression of both LEE- and non-LEE-encoded virulence factors in enteropathogenic and enterohemorrhagic Escherichia coli. Infect. Immun. 68, 61156126.

Elliott, S.J., Wainwright, L.A., McDaniel, T.K., Jarvis, K.G., Deng, Y.K., Lai, L.C., McNamara, B.P., Donnenberg, M.S., Kaper, J.B., 1998. The complete sequence of the locus of enterocyte effacement (LEE) from enteropathogenic Escherichia coli E2348/69. Mol. Microbiol. 28, 1-4.

Erdem, A.L., Avelino, F., Xicohtencatl-Cortes, J., Giron, J.A., 2007. Host Protein Binding and Adhesive Properties of H6 and H7 Flagella of Attaching and Effacing Escherichia coli. J. Bacteriol. 189, 7426-7435. doi:10.1128/JB.00464-07

Eric Cox, Vesna Melkebeek, Bert Devriendt, Bruno Goddeeris, Daisy Vanrompay, 2014. Vaccines Against Enteric E. coli Infections in Animals. doi:10.13140/2.1.3181.1526

Erickson, M.C., Doyle, M.P., 2007. Food as a vehicle for transmission of Shiga toxin-producing Escherichia coli. J. Food Prot. 70, 2426-2449.

Estrada-Garcia, T., Lopez-Saucedo, C., Thompson-Bonilla, R., Abonce, M., Lopez-Hernandez, D., Santos, J.I., Rosado, J.L., DuPont, H.L., Long, K.Z., 2009. Association of Diarrheagenic Escherichia coli Pathotypes with Infection and Diarrhea among Mexican Children and Association of Atypical Enteropathogenic E. coli with Acute Diarrhea. J. Clin. Microbiol. 47, 93-98. doi:10.1128/JCM.01166-08

Fegan, N., Vanderlinde, P., Higgs, G., Desmarchelier, P., 2004. The prevalence and concentration of Escherichia coli $\mathrm{O} 157$ in faeces of cattle from different production systems at slaughter. J. Appl. Microbiol. 97, 362-370. doi:10.1111/j.13652672.2004.02300.x

Fernandes, J., Su, W., Rahat-Rozenbloom, S., Wolever, T.M.S., Comelli, E.M., 2014. Adiposity, gut microbiota and faecal short chain fatty acids are linked in adult humans. Nutr.

Diabetes 4, e121. doi:10.1038/nutd.2014.23 
Flint, H.J., Bayer, E.A., Rincon, M.T., Lamed, R., White, B.A., 2008. Polysaccharide utilization by gut bacteria: potential for new insights from genomic analysis. Nat. Rev. Microbiol. 6, 121-131. doi:10.1038/nrmicro1817

Flores, M.-J., 2001. Impairment of lagging strand synthesis triggers the formation of a RuvABC substrate at replication forks. EMBO J. 20, 619-629. doi:10.1093/emboj/20.3.619

Foster, J.W., 2004. Escherichia coli acid resistance: tales of an amateur acidophile. Nat. Rev. Microbiol. 2, 898-907. doi:10.1038/nrmicro1021

Franceschini, S., Ceci, P., Alaleona, F., Chiancone, E., Ilari, A., 2006. Antioxidant Dps protein from the thermophilic cyanobacterium Thermosynechococcus elongatus. FEBS J. 273, 4913-4928. doi:10.1111/j.1742-4658.2006.05490.x

Frankel, G., Phillips, A.D., 2008. Attaching effacing Escherichia coli and paradigms of Tirtriggered actin polymerization: getting off the pedestal. Cell. Microbiol. 10, 549-556. doi:10.1111/j.1462-5822.2007.01103.x

Frankel, G., Phillips, A.D., Rosenshine, I., Dougan, G., Kaper, J.B., Knutton, S., 1998. Enteropathogenic and enterohaemorrhagic Escherichia coli: more subversive elements. Mol. Microbiol. 30, 911-921.

Franzin, F.M., Sircili, M.P., 2015. Locus of Enterocyte Effacement: A Pathogenicity Island Involved in the Virulence of Enteropathogenic and Enterohemorragic Escherichia coli Subjected to a Complex Network of Gene Regulation. BioMed Res. Int. 2015, 1-10. doi:10.1155/2015/534738

Friedberg, D., Umanski, T., Fang, Y., Rosenshine, I., 1999. Hierarchy in the expression of the locus of enterocyte effacement genes of enteropathogenic Escherichia coli. Mol. Microbiol. 34, 941-952.

Fukuda, S., Toh, H., Hase, K., Oshima, K., Nakanishi, Y., Yoshimura, K., Tobe, T., Clarke, J.M., Topping, D.L., Suzuki, T., Taylor, T.D., Itoh, K., Kikuchi, J., Morita, H., Hattori, M., Ohno, H., 2011. Bifidobacteria can protect from enteropathogenic infection through production of acetate. Nature 469, 543-547. doi:10.1038/nature09646

Galisteo, M., Duarte, J., Zarzuelo, A., 2008. Effects of dietary fibers on disturbances clustered in the metabolic syndrome. J. Nutr. Biochem. 19, 71-84. doi:10.1016/j.jnutbio.2007.02.009 
Garmendia, J., Frankel, G., Crepin, V.F., 2005. Enteropathogenic and enterohemorrhagic Escherichia coli infections: translocation, translocation, translocation. Infect. Immun. 73, 2573-2585. doi:10.1128/IAI.73.5.2573-2585.2005

Garsin, D.A., 2012. Ethanolamine: a signal to commence a host-associated lifestyle? mBio 3, e00172-112. doi:10.1128/mBio.00172-12

Gilbert, R.A., Tomkins, N., Padmanabha, J., Gough, J.M., Krause, D.O., McSweeney, C.S., 2005. Effect of finishing diets on Escherichia coli populations and prevalence of enterohaemorrhagic E. coli virulence genes in cattle faeces. J. Appl. Microbiol. 99, 885894. doi:10.1111/j.1365-2672.2005.02670.x

Goldman, M.J., Anderson, G.M., Stolzenberg, E.D., Kari, U.P., Zasloff, M., Wilson, J.M., 1997. Human beta-defensin-1 is a salt-sensitive antibiotic in lung that is inactivated in cystic fibrosis. Cell 88, 553-560.

Goldwater, P.N., Bettelheim, K.A., 2012. Treatment of enterohemorrhagic Escherichia coli (EHEC) infection and hemolytic uremic syndrome (HUS). BMC Med. 10, 12. doi:10.1186/1741-7015-10-12

Gong, S., Richard, H., Foster, J.W., 2003. YjdE (AdiC) is the arginine:agmatine antiporter essential for arginine-dependent acid resistance in Escherichia coli. J. Bacteriol. 185, 4402-4409.

Goodson, M., Rowbury, R.J., 1989. Resistance of acid-habituated Escherichia coli to organic acids and its medical and applied significance. Lett. Appl. Microbiol. 8, 211-214. doi:10.1111/j.1472-765X.1989.tb00250.x

Grant, R.A., Filman, D.J., Finkel, S.E., Kolter, R., Hogle, J.M., 1998. The crystal structure of Dps, a ferritin homolog that binds and protects DNA. Nat. Struct. Biol. 5, 294-303.

Groh, H., Schade, K., Hörhold-Schubert, C., 1993. Steroid metabolism with intestinal microorganisms. J. Basic Microbiol. 33, 59-72.

Gunderson, C.W., Segall, A.M., 2006. DNA repair, a novel antibacterial target: Holliday junction-trapping peptides induce DNA damage and chromosome segregation defects. Mol. Microbiol. 59, 1129-1148. doi:10.1111/j.1365-2958.2005.05009.x

Gyles, C.L., 2007. Shiga toxin-producing Escherichia coli: an overview. J. Anim. Sci. 85, E4562. doi:10.2527/jas.2006-508 
Haas, C.N., Thayyar-Madabusi, A., Rose, J.B., Gerba, C.P., 2000. Development of a doseresponse relationship for Escherichia coli O157:H7. Int. J. Food Microbiol. 56, 153-159. doi:10.1016/S0168-1605(99)00197-X

Halsey, T.A., Vazquez-Torres, A., Gravdahl, D.J., Fang, F.C., Libby, S.J., 2004. The FerritinLike Dps Protein Is Required for Salmonella enterica Serovar Typhimurium Oxidative Stress Resistance and Virulence. Infect. Immun. 72, 1155-1158. doi:10.1128/IAI.72.2.1155-1158.2004

Harig, J.M., Soergel, K.H., Komorowski, R.A., Wood, C.M., 1989. Treatment of diversion colitis with short-chain-fatty acid irrigation. N. Engl. J. Med. 320, 23-28. doi:10.1056/NEJM198901053200105

Hemrajani, C., Marches, O., Wiles, S., Girard, F., Dennis, A., Dziva, F., Best, A., Phillips, A.D., Berger, C.N., Mousnier, A., Crepin, V.F., Kruidenier, L., Woodward, M.J., Stevens, M.P., La Ragione, R.M., MacDonald, T.T., Frankel, G., 2008. Role of NleH, a type III secreted effector from attaching and effacing pathogens, in colonization of the bovine, ovine, and murine gut. Infect. Immun. 76, 4804-4813. doi:10.1128/IAI.00742-08

Hernandez-Doria, J.D., Sperandio, V., 2013. Nutrient and chemical sensing by intestinal pathogens. Microbes Infect. 15, 759-764. doi:10.1016/j.micinf.2013.06.013

Herold, S., Paton, J.C., Srimanote, P., Paton, A.W., 2009. Differential effects of short-chain fatty acids and iron on expression of iha in Shiga-toxigenic Escherichia coli. Microbiol. Read. Engl. 155, 3554-3563. doi:10.1099/mic.0.029454-0

Heroven, A.K., Böhme, K., Dersch, P., 2012. The Csr/Rsm system of Yersinia and related pathogens: a post-transcriptional strategy for managing virulence. RNA Biol. 9, 379-391. doi:10.4161/rna.19333

Higgins, L.M., Frankel, G., Douce, G., Dougan, G., MacDonald, T.T., 1999. Citrobacter rodentium infection in mice elicits a mucosal Th1 cytokine response and lesions similar to those in murine inflammatory bowel disease. Infect. Immun. 67, 3031-3039.

Ho, N.K., Henry, A.C., Johnson-Henry, K., Sherman, P.M., 2013. Pathogenicity, host responses and implications for management of enterohemorrhagic Escherichia coli O157:H7 infection. Can. J. Gastroenterol. J. Can. Gastroenterol. 27, 281-285.

Hoffmann, C., Hill, D.A., Minkah, N., Kirn, T., Troy, A., Artis, D., Bushman, F., 2009. Community-Wide Response of the Gut Microbiota to Enteropathogenic Citrobacter 
rodentium Infection Revealed by Deep Sequencing. Infect. Immun. 77, 4668-4678. doi:10.1128/IAI.00493-09

Hofmann, S.L., 1993. Southwestern Internal Medicine Conference: Shiga-like toxins in hemolytic-uremic syndrome and thrombotic thrombocytopenic purpura. Am. J. Med. Sci. 306, 398-406.

Horne, C., Vallance, B.A., Deng, W., Finlay, B.B., 2002. Current progress in enteropathogenic and enterohemorrhagic Escherichia coli vaccines. Expert Rev. Vaccines 1, 483-493. doi:10.1586/14760584.1.4.483

House, B., Kus, J.V., Prayitno, N., Mair, R., Que, L., Chingcuanco, F., Gannon, V., Cvitkovitch, D.G., Barnett Foster, D., 2009. Acid-stress-induced changes in enterohaemorrhagic Escherichia coli O157 : H7 virulence. Microbiol. Read. Engl. 155, 2907-2918. doi:10.1099/mic.0.025171-0

Huergo, L.F., Rahman, H., Ibrahimovic, A., Day, C.J., Korolik, V., 2013. Campylobacter jejuni Dps Protein Binds DNA in the Presence of Iron or Hydrogen Peroxide. J. Bacteriol. 195, 1970-1978. doi:10.1128/JB.00059-13

Hughes, K., Gillen, K., Semon, M., Karlinsey, J., 1993. Sensing structural intermediates in bacterial flagellar assembly by export of a negative regulator. Science 262, 1277-1280. doi: $10.1126 /$ science. 8235660

Ide, T., Laarmann, S., Greune, L., Schillers, H., Oberleithner, H., Schmidt, M.A., 2001. Characterization of translocation pores inserted into plasma membranes by type IIIsecreted Esp proteins of enteropathogenic Escherichia coli. Cell. Microbiol. 3, 669-679.

Ilari, A., Stefanini, S., Chiancone, E., Tsernoglou, D., 2000. The dodecameric ferritin from Listeria innocua contains a novel intersubunit iron-binding site. Nat. Struct. Biol. 7, 3843. doi:10.1038/71236

Islam, M.S., Stimson, W.H., 1990. Production and characterization of monoclonal antibodies with therapeutic potential against Shiga toxin. J. Clin. Lab. Immunol. 33, 11-16.

Iyer, R., Williams, C., Miller, C., 2003. Arginine-Agmatine Antiporter in Extreme Acid Resistance in Escherichia coli. J. Bacteriol. 185, 6556-6561. doi:10.1128/JB.185.22.6556-6561.2003

Jackson, M.P., 1990. Structure-function analyses of Shiga toxin and the Shiga-like toxins. Microb. Pathog. 8, 235-242. 
Jarboe, L.R., Royce, L.A., Liu, P., 2013. Understanding biocatalyst inhibition by carboxylic acids. Front. Microbiol. 4, 272. doi:10.3389/fmicb.2013.00272

Jeong, K., Hung, K., Baumler, D.J., Byrd, J.J., Kaspar, C.W., 2008. Acid stress damage of DNA is prevented by Dps binding in Escherichia coli O157:H7. BMC Microbiol. 8, 181. doi:10.1186/1471-2180-8-181

Jerse, A.E., Yu, J., Tall, B.D., Kaper, J.B., 1990. A genetic locus of enteropathogenic Escherichia coli necessary for the production of attaching and effacing lesions on tissue culture cells. Proc. Natl. Acad. Sci. U. S. A. 87, 7839-7843.

Kaper, J.B., Nataro, J.P., Mobley, H.L., 2004. Pathogenic Escherichia coli. Nat. Rev. Microbiol. 2, 123-140. doi:10.1038/nrmicro818

Karas, V.O., Westerlaken, I., Meyer, A.S., 2015. The DNA-Binding Protein from Starved Cells (Dps) Utilizes Dual Functions To Defend Cells against Multiple Stresses. J. Bacteriol. 197, 3206-3215. doi:10.1128/JB.00475-15

Karch, H., Schmidt, H., Janetzki-Mittmann, C., Scheef, J., Kröger, M., 1999. Shiga toxins even when different are encoded at identical positions in the genomes of related temperate bacteriophages. Mol. Gen. Genet. MGG 262, 600-607.

Karmali, M.A., 1989. Infection by verocytotoxin-producing Escherichia coli. Clin. Microbiol. Rev. 2, 15-38.

Karmali, M.A., Gannon, V., Sargeant, J.M., 2010. Verocytotoxin-producing Escherichia coli (VTEC). Vet. Microbiol. 140, 360-370. doi:10.1016/j.vetmic.2009.04.011

Karmali, M.A., Steele, B.T., Petric, M., Lim, C., 1983. Sporadic cases of haemolytic-uraemic syndrome associated with faecal cytotoxin and cytotoxin-producing Escherichia coli in stools. Lancet Lond. Engl. 1, 619-620.

Kasubuchi, M., Hasegawa, S., Hiramatsu, T., Ichimura, A., Kimura, I., 2015. Dietary Gut Microbial Metabolites, Short-chain Fatty Acids, and Host Metabolic Regulation. Nutrients 7, 2839-2849. doi:10.3390/nu7042839

Kelly, M., Hart, E., Mundy, R., Marchès, O., Wiles, S., Badea, L., Luck, S., Tauschek, M., Frankel, G., Robins-Browne, R.M., Hartland, E.L., 2006. Essential role of the type III secretion system effector NleB in colonization of mice by Citrobacter rodentium. Infect. Immun. 74, 2328-2337. doi:10.1128/IAI.74.4.2328-2337.2006 
Kenny, B., Abe, A., Stein, M., Finlay, B.B., 1997a. Enteropathogenic Escherichia coli protein secretion is induced in response to conditions similar to those in the gastrointestinal tract. Infect. Immun. 65, 2606-2612.

Kenny, B., DeVinney, R., Stein, M., Reinscheid, D.J., Frey, E.A., Finlay, B.B., 1997 b. Enteropathogenic E. coli (EPEC) transfers its receptor for intimate adherence into mammalian cells. Cell 91, 511-520.

Kepple, K.V., Boldt, J.L., Segall, A.M., 2005. Holliday junction-binding peptides inhibit distinct junction-processing enzymes. Proc. Natl. Acad. Sci. U. S. A. 102, 6867-6872. doi:10.1073/pnas.0409496102

Kepple, K.V., Patel, N., Salamon, P., Segall, A.M., 2008. Interactions between branched DNAs and peptide inhibitors of DNA repair. Nucleic Acids Res. 36, 5319-5334. doi:10.1093/nar/gkn512

Knutton, S., Baldwin, T., Williams, P.H., McNeish, A.S., 1989. Actin accumulation at sites of bacterial adhesion to tissue culture cells: basis of a new diagnostic test for enteropathogenic and enterohemorrhagic Escherichia coli. Infect. Immun. 57, 12901298.

Kohanski, M.A., Dwyer, D.J., Hayete, B., Lawrence, C.A., Collins, J.J., 2007. A Common Mechanism of Cellular Death Induced by Bactericidal Antibiotics. Cell 130, 797-810. doi:10.1016/j.cell.2007.06.049

Kurmanova, A., Llorente, A., Polesskaya, A., Garred, O., Olsnes, S., Kozlov, J., Sandvig, K., 2007. Structural requirements for furin-induced cleavage and activation of Shiga toxin. Biochem. Biophys. Res. Commun. 357, 144-149. doi:10.1016/j.bbrc.2007.03.110

Kus, J.V., Gebremedhin, A., Dang, V., Tran, S.-L., Serbanescu, A., Foster, D.B., 2011. Bile Salts Induce Resistance to Polymyxin in Enterohemorrhagic Escherichia coliO157:H7. J. Bacteriol. 193, 4509-4515. doi:10.1128/JB.00200-11

Lacroix, F.J., Cloeckaert, A., Grépinet, O., Pinault, C., Popoff, M.Y., Waxin, H., Pardon, P., 1996. Salmonella typhimurium acrB-like gene: identification and role in resistance to biliary salts and detergents and in murine infection. FEMS Microbiol. Lett. 135, 161167.

Lapeyraque, A.-L., Malina, M., Fremeaux-Bacchi, V., Boppel, T., Kirschfink, M., Oualha, M., Proulx, F., Clermont, M.-J., Le Deist, F., Niaudet, P., Schaefer, F., 2011. Eculizumab in 
severe Shiga-toxin-associated HUS. N. Engl. J. Med. 364, 2561-2563.

doi:10.1056/NEJMc1100859

Laursen, L., 2011. E. coli crisis opens door for Alexion drug trial. Nat. Biotechnol. 29, 671. doi:10.1038/nbt0811-671

Lawhon, S.D., Frye, J.G., Suyemoto, M., Porwollik, S., McClelland, M., Altier, C., 2003. Global regulation by CsrA in Salmonella typhimurium. Mol. Microbiol. 48, 1633-1645.

Lennen, R.M., Kruziki, M.A., Kumar, K., Zinkel, R.A., Burnum, K.E., Lipton, M.S., Hoover, S.W., Ranatunga, D.R., Wittkopp, T.M., Marner, W.D., Pfleger, B.F., 2011. Membrane stresses induced by overproduction of free fatty acids in Escherichia coli. Appl. Environ. Microbiol. 77, 8114-8128. doi:10.1128/AEM.05421-11

Lenz, A., Tomkins, J., Fabich, A.J., 2015. Draft Genome Sequence of Citrobacter rodentium DBS100 (ATCC 51459), a Primary Model of Enterohemorrhagic Escherichia coli Virulence. Genome Announc. 3, e00415-15. doi:10.1128/genomeA.00415-15

Lewis, S.B., Cook, V., Tighe, R., Schüller, S., 2015. Enterohemorrhagic Escherichia coli Colonization of Human Colonic Epithelium In Vitro and Ex Vivo. Infect. Immun. 83, 942-949. doi:10.1128/IAI.02928-14

Li, X., Rasko, D.A., Lockatell, C.V., Johnson, D.E., Mobley, H.L., 2001. Repression of bacterial motility by a novel fimbrial gene product. EMBO J. 20, 4854-4862. doi:10.1093/emboj/20.17.4854

Lin, J., Smith, M.P., Chapin, K.C., Baik, H.S., Bennett, G.N., Foster, J.W., 1996. Mechanisms of acid resistance in enterohemorrhagic Escherichia coli. Appl. Environ. Microbiol. 62, 3094-3100.

Lindahl, T., Nyberg, B., 1972. Rate of depurination of native deoxyribonucleic acid. Biochemistry (Mosc.) 11, 3610-3618. doi:10.1021/bi00769a018

Lingwood, C.A., Law, H., Richardson, S., Petric, M., Brunton, J.L., De Grandis, S., Karmali, M., 1987. Glycolipid binding of purified and recombinant Escherichia coli produced verotoxin in vitro. J. Biol. Chem. 262, 8834-8839.

Lino, M., Kus, J.V., Tran, S.L., Naqvi, Z., Binnington, B., Goodman, S.D., Segall, A.M., Foster, D.B., 2011. A novel antimicrobial peptide significantly enhances acid-induced killing of Shiga toxin-producing Escherichia coli O157 and non-O157 serotypes. Microbiol. Read. Engl. 157, 1768-1775. doi:10.1099/mic.0.047365-0 
Liu, X., Matsumura, P., 1994. The FlhD/FlhC complex, a transcriptional activator of the Escherichia coli flagellar class II operons. J. Bacteriol. 176, 7345-7351. doi:10.1128/jb.176.23.7345-7351.1994

Livak, K.J., Schmittgen, T.D., 2001. Analysis of relative gene expression data using real-time quantitative PCR and the 2(-Delta Delta C(T)) Method. Methods San Diego Calif 25, 402-408. doi:10.1006/meth.2001.1262

Lu, P., Ma, D., Chen, Y., Guo, Y., Chen, G.-Q., Deng, H., Shi, Y., 2013. L-glutamine provides acid resistance for Escherichia coli through enzymatic release of ammonia. Cell Res. 23, 635-644. doi:10.1038/cr.2013.13

Lupp, C., Robertson, M.L., Wickham, M.E., Sekirov, I., Champion, O.L., Gaynor, E.C., Finlay, B.B., 2007. Host-mediated inflammation disrupts the intestinal microbiota and promotes the overgrowth of Enterobacteriaceae. Cell Host Microbe 2, 119-129. doi:10.1016/j.chom.2007.06.010

Macfarlane, G.T., Gibson, G.R., Cummings, J.H., 1992. Comparison of fermentation reactions in different regions of the human colon. J. Appl. Bacteriol. 72, 57-64.

Macfarlane, S., Macfarlane, G.T., 2003. Regulation of short-chain fatty acid production. Proc. Nutr. Soc. 62, 67-72. doi:10.1079/PNS2002207

Mah, T.F., O'Toole, G.A., 2001. Mechanisms of biofilm resistance to antimicrobial agents. Trends Microbiol. 9, 34-39.

Mallick, E.M., McBee, M.E., Vanguri, V.K., Melton-Celsa, A.R., Schlieper, K., Karalius, B.J., O’Brien, A.D., Butterton, J.R., Leong, J.M., Schauer, D.B., 2012. A novel murine infection model for Shiga toxin-producing Escherichia coli. J. Clin. Invest. 122, 40124024. doi:10.1172/JCI62746

Malyukova, I., Murray, K.F., Zhu, C., Boedeker, E., Kane, A., Patterson, K., Peterson, J.R., Donowitz, M., Kovbasnjuk, O., 2009. Macropinocytosis in Shiga toxin 1 uptake by human intestinal epithelial cells and transcellular transcytosis. Am. J. Physiol. Gastrointest. Liver Physiol. 296, G78-92. doi:10.1152/ajpgi.90347.2008

Marlett, J.A., McBurney, M.I., Slavin, J.L., American Dietetic Association, 2002. Position of the American Dietetic Association: health implications of dietary fiber. J. Am. Diet. Assoc. $102,993-1000$. 
Martinez, A., Kolter, R., 1997. Protection of DNA during oxidative stress by the nonspecific DNA-binding protein Dps. J. Bacteriol. 179, 5188-5194.

Matsushiro, A., Sato, K., Miyamoto, H., Yamamura, T., Honda, T., 1999. Induction of prophages of enterohemorrhagic Escherichia coli O157:H7 with norfloxacin. J. Bacteriol. 181, 2257-2260.

McManus, M.C., 1997. Mechanisms of bacterial resistance to antimicrobial agents. Am. J. Health-Syst. Pharm. AJHP Off. J. Am. Soc. Health-Syst. Pharm. 54, 1420-1433-1446.

McNeilly, T.N., Mitchell, M.C., Rosser, T., McAteer, S., Low, J.C., Smith, D.G.E., Huntley, J.F., Mahajan, A., Gally, D.L., 2010. Immunization of cattle with a combination of purified intimin-531, EspA and Tir significantly reduces shedding of Escherichia coli O157:H7 following oral challenge. Vaccine 28, 1422-1428.

doi:10.1016/j.vaccine.2009.10.076

Mead, P.S., Griffin, P.M., 1998. Escherichia coli O157:H7. Lancet Lond. Engl. 352, 1207-1212. doi:10.1016/S0140-6736(98)01267-7

Mellies, J.L., Lorenzen, E., 2014. Enterohemorrhagic Escherichia coli Virulence Gene Regulation. Microbiol. Spectr. 2. doi:10.1128/microbiolspec.EHEC-0004-2013

Merritt, M.E., Donaldson, J.R., 2009. Effect of bile salts on the DNA and membrane integrity of enteric bacteria. J. Med. Microbiol. 58, 1533-1541. doi:10.1099/jmm.0.014092-0

Michel, B., Grompone, G., Flores, M.-J., Bidnenko, V., 2004. Multiple pathways process stalled replication forks. Proc. Natl. Acad. Sci. 101, 12783-12788.

doi:10.1073/pnas.0401586101

Momose, Y., Hirayama, K., Itoh, K., 2008. Effect of organic acids on inhibition of Escherichia coli $\mathrm{O} 157: \mathrm{H} 7$ colonization in gnotobiotic mice associated with infant intestinal microbiota. Antonie Van Leeuwenhoek 93, 141-149. doi:10.1007/s10482-007-9188-9

Moon, H.W., Whipp, S.C., Argenzio, R.A., Levine, M.M., Giannella, R.A., 1983. Attaching and effacing activities of rabbit and human enteropathogenic Escherichia coli in pig and rabbit intestines. Infect. Immun. 41, 1340-1351.

Morgan, J.K., Vendura, K.W., Stevens, S.M., Riordan, J.T., 2013. RcsB determines the locus of enterocyte effacement (LEE) expression and adherence phenotype of Escherichia coli O157 : H7 spinach outbreak strain TW14359 and coordinates bicarbonate-dependent LEE 
activation with repression of motility. Microbiol. Read. Engl. 159, 2342-2353.

doi:10.1099/mic.0.070201-0

Mukhopadhyay, S., Linstedt, A.D., 2012. Manganese blocks intracellular trafficking of Shiga toxin and protects against Shiga toxicosis. Science 335, 332-335.

doi:10.1126/science. 1215930

Mundy, R., MacDonald, T.T., Dougan, G., Frankel, G., Wiles, S., 2005. Citrobacter rodentium of mice and man. Cell. Microbiol. 7, 1697-1706. doi:10.1111/j.1462-5822.2005.00625.x

Mundy, R., Petrovska, L., Smollett, K., Simpson, N., Wilson, R.K., Yu, J., Tu, X., Rosenshine, I., Clare, S., Dougan, G., Frankel, G., 2004. Identification of a novel Citrobacter rodentium type III secreted protein, EspI, and roles of this and other secreted proteins in infection. Infect. Immun. 72, 2288-2302.

Murgas Torrazza, R., Neu, J., 2011. The developing intestinal microbiome and its relationship to health and disease in the neonate. J. Perinatol. Off. J. Calif. Perinat. Assoc. 31 Suppl 1, S29-34. doi:10.1038/jp.2010.172

Musso, G., Gambino, R., Cassader, M., 2011. Interactions between gut microbiota and host metabolism predisposing to obesity and diabetes. Annu. Rev. Med. 62, 361-380. doi:10.1146/annurev-med-012510-175505

Nair, S., Finkel, S.E., 2004. Dps Protects Cells against Multiple Stresses during Stationary Phase. J. Bacteriol. 186, 4192-4198. doi:10.1128/JB.186.13.4192-4198.2004

Nakanishi, N., Tashiro, K., Kuhara, S., Hayashi, T., Sugimoto, N., Tobe, T., 2009. Regulation of virulence by butyrate sensing in enterohaemorrhagic Escherichia coli. Microbiol. Read. Engl. 155, 521-530. doi:10.1099/mic.0.023499-0

Nataro, J.P., Kaper, J.B., 1998. Diarrheagenic Escherichia coli. Clin. Microbiol. Rev. 11, 142201.

Naylor, S.W., Flockhart, A., Nart, P., Smith, D.G.E., Huntley, J., Gally, D.L., Low, J.C., 2007. Shedding of Escherichia coli O157:H7 in Calves Is Reduced by Prior Colonization with the Homologous Strain. Appl. Environ. Microbiol. 73, 3765-3767.

doi:10.1128/AEM.02670-06

Nguyen, Y., Sperandio, V., 2012. Enterohemorrhagic E. coli (EHEC) pathogenesis. Front. Cell. Infect. Microbiol. 2. doi:10.3389/fcimb.2012.00090 
Nicholson, J.K., Holmes, E., Kinross, J., Burcelin, R., Gibson, G., Jia, W., Pettersson, S., 2012. Host-Gut Microbiota Metabolic Interactions. Science 336, 1262-1267. doi:10.1126/science. 1223813

Njoroge, J., Sperandio, V., 2012. Enterohemorrhagic Escherichia coli virulence regulation by two bacterial adrenergic kinases, QseC and QseE. Infect. Immun. 80, 688-703. doi:10.1128/IAI.05921-11

O’Brien, A.D., Tesh, V.L., Donohue-Rolfe, A., Jackson, M.P., Olsnes, S., Sandvig, K., Lindberg, A.A., Keusch, G.T., 1992. Shiga toxin: biochemistry, genetics, mode of action, and role in pathogenesis. Curr. Top. Microbiol. Immunol. 180, 65-94.

Obrig, T.G., 2010. Escherichia coli Shiga Toxin Mechanisms of Action in Renal Disease. Toxins 2, 2769-2794. doi:10.3390/toxins2122769

Orchard, S.S., Rostron, J.E., Segall, A.M., 2012. Escherichia coli enterobactin synthesis and uptake mutants are hypersensitive to an antimicrobial peptide that limits the availability of iron in addition to blocking Holliday junction resolution. Microbiol. Read. Engl. 158, 547-559. doi:10.1099/mic.0.054361-0

Pankey, G.A., Sabath, L.D., 2004. Clinical relevance of bacteriostatic versus bactericidal mechanisms of action in the treatment of Gram-positive bacterial infections. Clin. Infect. Dis. Off. Publ. Infect. Dis. Soc. Am. 38, 864-870. doi:10.1086/381972

Papapietro, O., Teatero, S., Thanabalasuriar, A., Yuki, K.E., Diez, E., Zhu, L., Kang, E., Dhillon, S., Muise, A.M., Durocher, Y., Marcinkiewicz, M.M., Malo, D., Gruenheid, S., 2013. RSpondin 2 signalling mediates susceptibility to fatal infectious diarrhoea. Nat. Commun. 4, 1898. doi:10.1038/ncomms 2816

Paton, J.C., Paton, A.W., 1998. Pathogenesis and diagnosis of Shiga toxin-producing Escherichia coli infections. Clin. Microbiol. Rev. 11, 450-479.

Persad, A.K., LeJeune, J.T., 2014. Animal Reservoirs of Shiga Toxin-Producing Escherichia coli. Microbiol. Spectr. 2. doi:10.1128/microbiolspec.EHEC-0027-2014

Petty, N.K., Bulgin, R., Crepin, V.F., Cerdeno-Tarraga, A.M., Schroeder, G.N., Quail, M.A., Lennard, N., Corton, C., Barron, A., Clark, L., Toribio, A.L., Parkhill, J., Dougan, G., Frankel, G., Thomson, N.R., 2010. The Citrobacter rodentium Genome Sequence Reveals Convergent Evolution with Human Pathogenic Escherichia coli. J. Bacteriol. 192, 525538. doi:10.1128/JB.01144-09 
Pfaffl, M.W., 2001. A new mathematical model for relative quantification in real-time RT-PCR. Nucleic Acids Res. 29, e45.

Pifer, R., Sperandio, V., 2014. The Interplay between the Microbiota and Enterohemorrhagic Escherichia coli. Microbiol. Spectr. 2. doi:10.1128/microbiolspec.EHEC-0015-2013 Pruimboom-Brees, I.M., Morgan, T.W., Ackermann, M.R., Nystrom, E.D., Samuel, J.E., Cornick, N.A., Moon, H.W., 2000. Cattle lack vascular receptors for Escherichia coli O157:H7 Shiga toxins. Proc. Natl. Acad. Sci. U. S. A. 97, 10325-10329. doi:10.1073/pnas.190329997

Qin, J., Li, R., Raes, J., Arumugam, M., Burgdorf, K.S., Manichanh, C., Nielsen, T., Pons, N., Levenez, F., Yamada, T., Mende, D.R., Li, J., Xu, J., Li, S., Li, D., Cao, J., Wang, B., Liang, H., Zheng, H., Xie, Y., Tap, J., Lepage, P., Bertalan, M., Batto, J.-M., Hansen, T., Le Paslier, D., Linneberg, A., Nielsen, H.B., Pelletier, E., Renault, P., Sicheritz-Ponten, T., Turner, K., Zhu, H., Yu, C., Li, S., Jian, M., Zhou, Y., Li, Y., Zhang, X., Li, S., Qin, N., Yang, H., Wang, J., Brunak, S., Doré, J., Guarner, F., Kristiansen, K., Pedersen, O., Parkhill, J., Weissenbach, J., Antolin, M., Artiguenave, F., Blottiere, H., Borruel, N., Bruls, T., Casellas, F., Chervaux, C., Cultrone, A., Delorme, C., Denariaz, G., Dervyn, R., Forte, M., Friss, C., van de Guchte, M., Guedon, E., Haimet, F., Jamet, A., Juste, C., Kaci, G., Kleerebezem, M., Knol, J., Kristensen, M., Layec, S., Le Roux, K., Leclerc, M., Maguin, E., Melo Minardi, R., Oozeer, R., Rescigno, M., Sanchez, N., Tims, S., Torrejon, T., Varela, E., de Vos, W., Winogradsky, Y., Zoetendal, E., Bork, P., Ehrlich, S.D., Wang, J., 2010. A human gut microbial gene catalogue established by metagenomic sequencing. Nature 464, 59-65. doi:10.1038/nature08821

Rahal, E.A., Kazzi, N., Nassar, F.J., Matar, G.M., 2012. Escherichia coli O157:H7—Clinical aspects and novel treatment approaches. Front. Cell. Infect. Microbiol. 2. doi:10.3389/fcimb.2012.00138

Raja, N., Goodson, M., Smith, D.G., Rowbury, R.J., 1991. Decreased DNA damage by acid and increased repair of acid-damaged DNA in acid-habituated Escherichia coli. J. Appl. Bacteriol. 70, 507-511.

Ravel, J., Gajer, P., Abdo, Z., Schneider, G.M., Koenig, S.S.K., McCulle, S.L., Karlebach, S., Gorle, R., Russell, J., Tacket, C.O., Brotman, R.M., Davis, C.C., Ault, K., Peralta, L., 
Forney, L.J., 2011. Vaginal microbiome of reproductive-age women. Proc. Natl. Acad. Sci. U. S. A. 108 Suppl 1, 4680-4687. doi:10.1073/pnas.1002611107

Rendón, M.A., Saldaña, Z., Erdem, A.L., Monteiro-Neto, V., Vázquez, A., Kaper, J.B., Puente, J.L., Girón, J.A., 2007. Commensal and pathogenic Escherichia coli use a common pilus adherence factor for epithelial cell colonization. Proc. Natl. Acad. Sci. U. S. A. 104, 10637-10642. doi:10.1073/pnas.0704104104

Richard, H., Foster, J.W., 2004. Escherichia coli Glutamate- and Arginine-Dependent Acid Resistance Systems Increase Internal $\mathrm{pH}$ and Reverse Transmembrane Potential. J. Bacteriol. 186, 6032-6041. doi:10.1128/JB.186.18.6032-6041.2004

Ridlon, J.M., Kang, D.-J., Hylemon, P.B., 2006. Bile salt biotransformations by human intestinal bacteria. J. Lipid Res. 47, 241-259. doi:10.1194/j1r.R500013-JLR200

Riley, L.W., Remis, R.S., Helgerson, S.D., McGee, H.B., Wells, J.G., Davis, B.R., Hebert, R.J., Olcott, E.S., Johnson, L.M., Hargrett, N.T., Blake, P.A., Cohen, M.L., 1983. Hemorrhagic colitis associated with a rare Escherichia coli serotype. N. Engl. J. Med. 308, 681-685. doi:10.1056/NEJM198303243081203

Ritchie, J.M., Waldor, M.K., 2005. The locus of enterocyte effacement-encoded effector proteins all promote enterohemorrhagic Escherichia coli pathogenicity in infant rabbits. Infect. Immun. 73, 1466-1474. doi:10.1128/IAI.73.3.1466-1474.2005

Rodrigues, D.M., Sousa, A.J., Johnson-Henry, K.C., Sherman, P.M., Gareau, M.G., 2012. Probiotics are effective for the prevention and treatment of Citrobacter rodentiuminduced colitis in mice. J. Infect. Dis. 206, 99-109. doi:10.1093/infdis/jis177

Rodríguez-Moyá, M., Gonzalez, R., 2015. Proteomic analysis of the response of Escherichia coli to short-chain fatty acids. J. Proteomics 122, 86-99. doi:10.1016/j.jprot.2015.03.033

Roediger, W.E., 1982. Utilization of nutrients by isolated epithelial cells of the rat colon. Gastroenterology 83, 424-429.

Rossez, Y., Wolfson, E.B., Holmes, A., Gally, D.L., Holden, N.J., 2015. Bacterial Flagella: Twist and Stick, or Dodge across the Kingdoms. PLOS Pathog. 11, e1004483. doi:10.1371/journal.ppat.1004483

Roy, C.C., Kien, C.L., Bouthillier, L., Levy, E., 2006. Short-chain fatty acids: ready for prime time? Nutr. Clin. Pract. Off. Publ. Am. Soc. Parenter. Enter. Nutr. 21, 351-366. 
Royall, D., Wolever, T.M., Jeejeebhoy, K.N., 1990. Clinical significance of colonic fermentation. Am. J. Gastroenterol. 85, 1307-1312.

Ruppin, H., Bar-Meir, S., Soergel, K.H., Wood, C.M., Schmitt, M.G., 1980. Absorption of shortchain fatty acids by the colon. Gastroenterology 78, 1500-1507.

Samuel, B.S., Shaito, A., Motoike, T., Rey, F.E., Backhed, F., Manchester, J.K., Hammer, R.E., Williams, S.C., Crowley, J., Yanagisawa, M., Gordon, J.I., 2008. Effects of the gut microbiota on host adiposity are modulated by the short-chain fatty-acid binding $\mathrm{G}$ protein-coupled receptor, Gpr41. Proc. Natl. Acad. Sci. U. S. A. 105, 16767-16772. doi:10.1073/pnas.0808567105

Sandvig, K., 2001. Shiga toxins. Toxicon Off. J. Int. Soc. Toxinology 39, 1629-1635.

Sandvig, K., van Deurs, B., 1996. Endocytosis, intracellular transport, and cytotoxic action of Shiga toxin and ricin. Physiol. Rev. 76, 949-966.

Saxena, T., Kaushik, P., Krishna Mohan, M., 2015. Prevalence of E. coli O157:H7 in water sources: an overview on associated diseases, outbreaks and detection methods. Diagn. Microbiol. Infect. Dis. 82, 249-264. doi:10.1016/j.diagmicrobio.2015.03.015

Schüller, S., Phillips, A.D., 2010. Microaerobic conditions enhance type III secretion and adherence of enterohaemorrhagic Escherichia coli to polarized human intestinal epithelial cells. Environ. Microbiol. 12, 2426-2435. doi:10.1111/j.1462-2920.2010.02216.x

Sekiya, K., Ohishi, M., Ogino, T., Tamano, K., Sasakawa, C., Abe, A., 2001. Supermolecular structure of the enteropathogenic Escherichia coli type III secretion system and its direct interaction with the EspA-sheath-like structure. Proc. Natl. Acad. Sci. U. S. A. 98, 11638-11643. doi:10.1073/pnas.191378598

Serna, A., Boedeker, E.C., 2008. Pathogenesis and treatment of Shiga toxin-producing Escherichia coli infections. Curr. Opin. Gastroenterol. 24, 38-47. doi:10.1097/MOG.0b013e3282f2dfb8

Sharples, G.J., 2001. The X philes: structure-specific endonucleases that resolve Holliday junctions. Mol. Microbiol. 39, 823-834.

Sharples, G.J., Ingleston, S.M., Lloyd, R.G., 1999. Holliday junction processing in bacteria: insights from the evolutionary conservation of RuvABC, RecG, and RusA. J. Bacteriol. $181,5543-5550$. 
Sherman, P.M., Johnson-Henry, K.C., Yeung, H.P., Ngo, P.S.C., Goulet, J., Tompkins, T.A., 2005. Probiotics reduce enterohemorrhagic Escherichia coli O157:H7- and enteropathogenic E. coli O127:H6-induced changes in polarized T84 epithelial cell monolayers by reducing bacterial adhesion and cytoskeletal rearrangements. Infect. Immun. 73, 5183-5188. doi:10.1128/IAI.73.8.5183-5188.2005

Smith, A., Bhagwat, A.A., 2013. Hypervirulent-host-associated Citrobacter rodentium cells have poor acid tolerance. Curr. Microbiol. 66, 522-526. doi:10.1007/s00284-012-0298-x

Smith, C.J., Bryant, M.P., 1979. Introduction to metabolic activities of intestinal bacteria. Am. J. Clin. Nutr. 32, 149-157.

Smith, D.R., 2014. Vaccination of Cattle against Escherichia coli O157:H7. Microbiol. Spectr. 2. doi:10.1128/microbiolspec.EHEC-0006-2013

Smith, J.L., 2003. The role of gastric acid in preventing foodborne disease and how bacteria overcome acid conditions. J. Food Prot. 66, 1292-1303.

Sperandio, V., Mellies, J.L., Nguyen, W., Shin, S., Kaper, J.B., 1999. Quorum sensing controls expression of the type III secretion gene transcription and protein secretion in enterohemorrhagic and enteropathogenic Escherichia coli. Proc. Natl. Acad. Sci. U. S. A. 96, 15196-15201.

Sperandio, V., Torres, A.G., Jarvis, B., Nataro, J.P., Kaper, J.B., 2003. Bacteria-host communication: the language of hormones. Proc. Natl. Acad. Sci. U. S. A. 100, 89518956. doi:10.1073/pnas. 1537100100

Ståhl, A.-L., Karpman, D., 2014. Enterohemorrhagic Escherichia coli Pathogenesis and the Host Response. Microbiol. Spectr. 2. doi:10.1128/microbiolspec.EHEC-0009-2013

Stewart, P.S., Costerton, J.W., 2001. Antibiotic resistance of bacteria in biofilms. Lancet Lond. Engl. 358, 135-138.

Su, L.Y., Willner, D.L., Segall, A.M., 2010. An antimicrobial peptide that targets DNA repair intermediates in vitro inhibits Salmonella growth within murine macrophages. Antimicrob. Agents Chemother. 54, 1888-1899. doi:10.1128/AAC.01610-09

Sun, Y., O’Riordan, M.X.D., 2013. Regulation of Bacterial Pathogenesis by Intestinal ShortChain Fatty Acids, in: Advances in Applied Microbiology. Elsevier, pp. 93-118. 
Suzuki, K., Wang, X., Weilbacher, T., Pernestig, A.-K., Melefors, O., Georgellis, D., Babitzke, P., Romeo, T., 2002. Regulatory circuitry of the CsrA/CsrB and BarA/UvrY systems of Escherichia coli. J. Bacteriol. 184, 5130-5140.

Swann, J.R., Want, E.J., Geier, F.M., Spagou, K., Wilson, I.D., Sidaway, J.E., Nicholson, J.K., Holmes, E., 2011. Systemic gut microbial modulation of bile acid metabolism in host tissue compartments. Proc. Natl. Acad. Sci. U. S. A. 108 Suppl 1, 4523-4530. doi:10.1073/pnas.1006734107

Swenson, G.J., Stochastic, J., Bolander, F.F., Long, R.A., 2012. Acid stress response in environmental and clinical strains of enteric bacteria. Front. Biol. 7, 495-505. doi:10.1007/s11515-012-1191-5

Takao, M., Yen, H., Tobe, T., 2014. LeuO enhances butyrate-induced virulence expression through a positive regulatory loop in enterohaemorrhagic Escherichia coli. Mol. Microbiol. 93, 1302-1313. doi:10.1111/mmi.12737

Tam, J.P., 2002. Correlations of Cationic Charges with Salt Sensitivity and Microbial Specificity of Cystine-stabilized beta -Strand Antimicrobial Peptides. J. Biol. Chem. 277, 5045050456. doi:10.1074/jbc.M208429200

Tennant, S.M., Hartland, E.L., Phumoonna, T., Lyras, D., Rood, J.I., Robins-Browne, R.M., van Driel, I.R., 2008. Influence of Gastric Acid on Susceptibility to Infection with Ingested Bacterial Pathogens. Infect. Immun. 76, 639-645. doi:10.1128/IAI.01138-07

Tenover, F.C., 2006. Mechanisms of antimicrobial resistance in bacteria. Am. J. Med. 119, S310-70. doi:10.1016/j.amjmed.2006.03.011

Thompson, S.A., Latch, R.L., Blaser, M.J., 1998. Molecular characterization of the Helicobacter pylori uvrB gene. Gene 209, 113-122. doi:10.1016/S0378-1119(98)00028-6

Tobe, T., Beatson, S.A., Taniguchi, H., Abe, H., Bailey, C.M., Fivian, A., Younis, R., Matthews, S., Marches, O., Frankel, G., Hayashi, T., Pallen, M.J., 2006. An extensive repertoire of type III secretion effectors in Escherichia coli O157 and the role of lambdoid phages in their dissemination. Proc. Natl. Acad. Sci. U. S. A. 103, 14941-14946. doi:10.1073/pnas.0604891103

Tobe, T., Nakanishi, N., Sugimoto, N., 2011. Activation of Motility by Sensing Short-Chain Fatty Acids via Two Steps in a Flagellar Gene Regulatory Cascade in Enterohemorrhagic Escherichia coli. Infect. Immun. 79, 1016-1024. doi:10.1128/IAI.00927-10 
Topping, D.L., Clifton, P.M., 2001. Short-chain fatty acids and human colonic function: roles of resistant starch and nonstarch polysaccharides. Physiol. Rev. 81, 1031-1064.

Toshima, H., Yoshimura, A., Arikawa, K., Hidaka, A., Ogasawara, J., Hase, A., Masaki, H., Nishikawa, Y., 2007. Enhancement of Shiga toxin production in enterohemorrhagic Escherichia coli serotype O157:H7 by DNase colicins. Appl. Environ. Microbiol. 73, 7582-7588. doi:10.1128/AEM.01326-07

Tremaroli, V., Bäckhed, F., 2012. Functional interactions between the gut microbiota and host metabolism. Nature 489, 242-249. doi:10.1038/nature1 1552

Turnbaugh, P.J., Hamady, M., Yatsunenko, T., Cantarel, B.L., Duncan, A., Ley, R.E., Sogin, M.L., Jones, W.J., Roe, B.A., Affourtit, J.P., Egholm, M., Henrissat, B., Heath, A.C., Knight, R., Gordon, J.I., 2009. A core gut microbiome in obese and lean twins. Nature 457, 480-484. doi:10.1038/nature07540

Ursell, L.K., Metcalf, J.L., Parfrey, L.W., Knight, R., 2012. Defining the human microbiome. Nutr. Rev. 70, S38-S44. doi:10.1111/j.1753-4887.2012.00493.x

Van Deun, K., Pasmans, F., Van Immerseel, F., Ducatelle, R., Haesebrouck, F., 2008. Butyrate protects Caco-2 cells from Campylobacter jejuni invasion and translocation. Br. J. Nutr. 100, 480-484. doi:10.1017/S0007114508921693

van Hoek, M.J., Merks, R.M., 2012. Redox balance is key to explaining full vs. partial switching to low-yield metabolism. BMC Syst. Biol. 6, 22. doi:10.1186/1752-0509-6-22

Venn, B.J., Mann, J.I., 2004. Cereal grains, legumes and diabetes. Eur. J. Clin. Nutr. 58, 14431461. doi:10.1038/sj.ejcn.1601995

Von Döhren, H., 2009. Antibiotics: Actions, origins, resistance, by C. Walsh. 2003. Washington, DC: ASM Press. 345 pp. \$99.95 (hardcover). Protein Sci. 13, 3059-3060. doi:10.1110/ps.041032204

Walsh, C., 2000. Molecular mechanisms that confer antibacterial drug resistance. Nature 406, 775-781. doi:10.1038/35021219

Wang, X., Dubey, A.K., Suzuki, K., Baker, C.S., Babitzke, P., Romeo, T., 2005. CsrA posttranscriptionally represses pga $\mathrm{ABCD}$, responsible for synthesis of a biofilm polysaccharide adhesin of Escherichia coli. Mol. Microbiol. 56, 1648-1663. doi:10.1111/j.1365-2958.2005.04648.x 
Wei, B.L., Brun-Zinkernagel, A.M., Simecka, J.W., Prüss, B.M., Babitzke, P., Romeo, T., 2001. Positive regulation of motility and flhDC expression by the RNA-binding protein CsrA of Escherichia coli. Mol. Microbiol. 40, 245-256.

Weilbacher, T., Suzuki, K., Dubey, A.K., Wang, X., Gudapaty, S., Morozov, I., Baker, C.S., Georgellis, D., Babitzke, P., Romeo, T., 2003. A novel sRNA component of the carbon storage regulatory system of Escherichia coli. Mol. Microbiol. 48, 657-670.

Weiss, S.M., Ladwein, M., Schmidt, D., Ehinger, J., Lommel, S., Städing, K., Beutling, U., Disanza, A., Frank, R., Jänsch, L., Scita, G., Gunzer, F., Rottner, K., Stradal, T.E.B., 2009. IRSp53 links the enterohemorrhagic E. coli effectors Tir and EspFU for actin pedestal formation. Cell Host Microbe 5, 244-258. doi:10.1016/j.chom.2009.02.003

Whitby, M.C., Lloyd, R.G., 1998. Targeting Holliday junctions by the RecG branch migration protein of Escherichia coli. J. Biol. Chem. 273, 19729-19739.

Whitby, M.C., Ryder, L., Lloyd, R.G., 1993. Reverse branch migration of holliday junctions by RecG protein: A new mechanism for resolution of intermediates in recombination and DNA repair. Cell 75, 341-350. doi:10.1016/0092-8674(93)80075-P

Wibbenmeyer, J.A., Provenzano, D., Landry, C.F., Klose, K.E., Delcour, A.H., 2002. Vibrio cholerae OmpU and OmpT porins are differentially affected by bile. Infect. Immun. 70, $121-126$.

Widiasih, D.A., Ido, N., Omoe, K., Sugii, S., Shinagawa, K., 2004. Duration and magnitude of faecal shedding of Shiga toxin-producing Escherichia coli from naturally infected cattle. Epidemiol. Infect. 132, 67-75. doi:10.1017/S0950268803001468

Wiles, S., Clare, S., Harker, J., Huett, A., Young, D., Dougan, G., Frankel, G., 2004. Organ specificity, colonization and clearance dynamics in vivo following oral challenges with the murine pathogen Citrobacter rodentium. Cell. Microbiol. 6, 963-972. doi:10.1111/j.1462-5822.2004.00414.X

Wiles, S., Hanage, W.P., Frankel, G., Robertson, B., 2006a. Modelling infectious disease — time to think outside the box? Nat. Rev. Microbiol. 4, 307-312. doi:10.1038/nrmicro1386

Wiles, S., Pickard, K.M., Peng, K., MacDonald, T.T., Frankel, G., 2006b. In vivo bioluminescence imaging of the murine pathogen Citrobacter rodentium. Infect. Immun. 74, 5391-5396. doi:10.1128/IAI.00848-06 
Wolf, S.G., Frenkiel, D., Arad, T., Finkel, S.E., Kolter, R., Minsky, A., 1999. DNA protection by stress-induced biocrystallization. Nature 400, 83-85. doi:10.1038/21918

Wong, C.S., Mooney, J.C., Brandt, J.R., Staples, A.O., Jelacic, S., Boster, D.R., Watkins, S.L., Tarr, P.I., 2012. Risk factors for the hemolytic uremic syndrome in children infected with Escherichia coli O157:H7: a multivariable analysis. Clin. Infect. Dis. Off. Publ. Infect. Dis. Soc. Am. 55, 33-41. doi:10.1093/cid/cis299

Wong, J.M.W., de Souza, R., Kendall, C.W.C., Emam, A., Jenkins, D.J.A., 2006. Colonic health: fermentation and short chain fatty acids. J. Clin. Gastroenterol. 40, 235-243.

Yin, X., Feng, Y., Wheatcroft, R., Chambers, J., Gong, J., Gyles, C.L., 2011. Adherence of Escherichia coli O157:H7 to epithelial cells in vitro and in pig gut loops is affected by bacterial culture conditions. Can. J. Vet. Res. Rev. Can. Rech. Veterinaire 75, 81-88.

Zeth, K., 2012. Dps biomineralizing proteins: multifunctional architects of nature. Biochem. J. 445, 297-311. doi:10.1042/BJ20120514

Zhao, G., Ceci, P., Ilari, A., Giangiacomo, L., Laue, T.M., Chiancone, E., Chasteen, N.D., 2002. Iron and hydrogen peroxide detoxification properties of DNA-binding protein from starved cells. A ferritin-like DNA-binding protein of Escherichia coli. J. Biol. Chem. 277, 27689-27696. doi:10.1074/jbc.M202094200 